\title{
CHARACTERIZING COSEISMIC IONOSPHERIC DISTURBANCE FOR SURFACE-RUPTURING EARTHQUAKES
}

by

Rebekah Faith Lee

\author{
A thesis \\ submitted in partial fulfillment \\ of the requirements for the degree of \\ Master of Science in Geophysics \\ Boise State University
}

December 2017 
(C) 2017

Rebekah Faith Lee

ALL RIGHTS RESERVED 
BOISE STATE UNIVERSITY GRADUATE COLLEGE

\title{
DEFENSE COMMITTEE AND FINAL READING APPROVALS
}

\author{
of the thesis submitted by
}

\author{
Rebekah Faith Lee
}

Thesis Title: Characterizing Coseismic Ionospheric Disturbance for SurfaceRupturing Earthquakes

Date of Final Oral Examination: 25 October 2017

The following individuals read and discussed the dissertation submitted by student Rebekah Faith Lee, and they evaluated the student's presentation and response to questions during the final oral examination. They found that the student passed the final oral examination.

Dylan Mikesell Ph.D. Chair, Supervisory Committee

Jeffrey B. Johnson Ph.D. Member, Supervisory Committee

Lee M. Liberty M.S. Member, Supervisory Committee

The final reading approval of the thesis was granted by Dylan Mikesell Ph.D., Chair of the Supervisory Committee. The thesis was approved by the Graduate College. 


\section{DEDICATION}

To my parents. Who have always supported me in everything I do. 


\section{ACKNOWLEDGMENT}

Thanks first and especially to my advisor, Dr. T. Dylan Mikesell for taking me on as one of your first students. I couldn't have asked for a better advisor. Thank you for all your patient help throughout the past few years. Thanks also to Dr. Lucie M. Rolland for hosting me for two weeks in France to learn about extracting TEC and for all your continued help thereafter. Thanks to both Lucie and Dylan for the privilege to write a paper with you. Thanks to GSA for the research grants that made travel to France possible. Thanks to my committee for all your help. Thanks to Dr. Hunter A. Knox for introducing me to Dylan. Finally, thank you to everyone in the Geosciences department at Boise State University for providing such a collaborative and supportive work environment. 


\begin{abstract}
Coseismic ionospheric disturbances (CID) are commonly identified using global navigation space system (GNSS) satellites. Little research, however, has focused on using total electron content (TEC) observations to characterize acoustic sources on Earth's surface. For this thesis, I investigate the applicability of an analytical method to invert the TEC for the acoustic wave. The inversion is based on the modeling of a transfer function. Deconvolving the TEC by the transfer function gives the acoustic wave. Inverting for the acoustic wave in this way would remove phase differences in the TEC created by atmospheric-ionospheric coupling. I test the assumption in the model of a 1D, vertically varying ionosphere by comparing numerical models of the TEC using 1D and 3D electron density divergences. I find the results are complex and recommend obtaining a transfer function that includes a 3D ionosphere. Regardless, even with the phase shift introduced by ionospheric coupling, we are able to apply seismic methods to the TEC.

I show an example of applying seismic methods to the TEC of the 2016 Kaikoura earthquake. In this chapter, I highlight the ionospheric response to the rupture. I use numerical modeling and find the TEC response to be more consistent with an acoustic source located northeast of the initial rupture. I also apply backprojection to the TEC for the first time and obtain a source just northwest of the rupture area. The errors in the backprojection are consistent with expected errors from local winds,
\end{abstract}


which were not included in the model. Besides accounting for local winds in future work, inversion of the acoustic wave should also improve backprojection results by removing phase differences in the TEC. 


\section{TABLE OF CONTENTS}

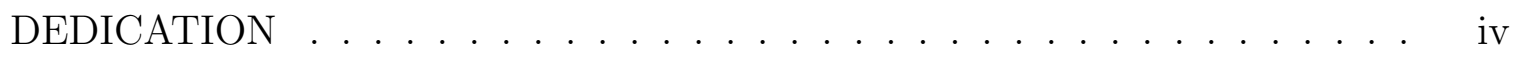

ACKNOWLEDGMENT ....................

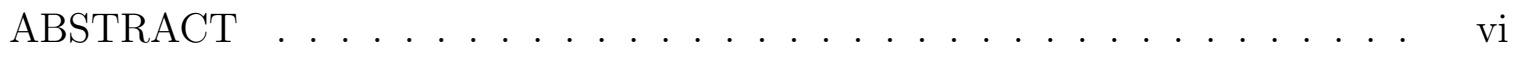

LIST OF FIGURES $\ldots \ldots \ldots \ldots \ldots \ldots \ldots \ldots \ldots \ldots \ldots \ldots \ldots$

LIST OF TABLES .................... . . xx

1 INTRODUCTION \& RESEARCH OBJECTIVE . . . . . . . . . . . 1

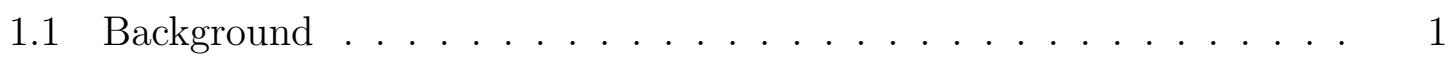

1.1 .1 The Ionosphere . . . . . . . . . . . . . . . . . . . 1

1.1.2 Total Electron Content . . . . . . . . . . . . . . . . . 2

1.1.3 Ionospheric Pierce Point . . . . . . . . . . . . . . 3

1.2 Identifying CIDs $\ldots \ldots \ldots \ldots \ldots \ldots \ldots \ldots \ldots$

1.2.1 TEC Time Series . . . . . . . . . . . . . . 6

1.2 .2 Modeling . . . . . . . . . . . . . . . . . . 11

1.3 Source Information in TEC $\ldots \ldots \ldots \ldots$

1.4 Significance . . . . . . . . . . . . . . . . . . . . . 14

1.5 Research Objective . . . . . . . . . . . . . . . . . 14 


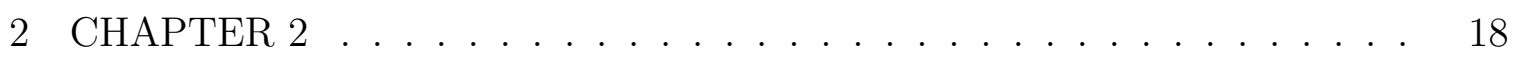

2.1 Chapter Summary . . . . . . . . . . . . . . . . . . . . . . . 18

2.2 Numerical Method . . . . . . . . . . . . . . . . . . . 18

2.2 .1 Acoustic Wave . . . . . . . . . . . . . . . . . . . . 18

2.2 .2 Ionospheric Coupling . . . . . . . . . . . . . . . . . 20

2.2 .3 Line of Sight Integration . . . . . . . . . . . . . . . . 23

2.3 Analytical Model . . . . . . . . . . . . . . . . . . . . . 24

2.3.1 Acoustic Wave: Plane Wave Approximation . . . . . . . . . 24

2.3.2 Ionospheric Coupling . . . . . . . . . . . . . . . . . . . 24

2.3 .3 Line of Sight Integration . . . . . . . . . . . . . . 25

3 TEC SENSITIVITY TO MODEL PARAMETERS . . . . . . . . . . . 27

3.1 Chapter Summary . . . . . . . . . . . . . . . . . . . 27

3.2 Methods . . . . . . . . . . . . . . . . . . . . 28

3.2 .1 Station Grid . . . . . . . . . . . . . . . 28

$3.2 .2 \quad$ Grid Locations . . . . . . . . . . . . . . . . . . . . . . . 29

$3.2 .3 \quad$ Satellite Positions . . . . . . . . . . . . . . . . . . . . . . . 29

3.3 Results . . . . . . . . . . . . . . . . . . . . . . . 31

3.3.1 Impact of Electron Density Divergence on TEC . . . . . . . 31

3.3.2 Magnetic Field Influence on TEC . . . . . . . . . . . . . 32

3.4 Discussion . . . . . . . . . . . . . . . . . . . . . . . . . 39

3.5 Conclusion . . . . . . . . . . . . . . . . . . . . 41

4 TEC EXTRACTION . . . . . . . . . . . . . . . . . . . 42

4.1 Download GNSS data . . . . . . . . . . . . . . . . . . 42 
$4.2 \quad$ TEC Extraction . . . . . . . . . . . . . . . . . . 43

4.2 .1 Workflow Example . . . . . . . . . . . . . . . 45

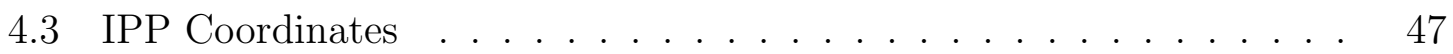

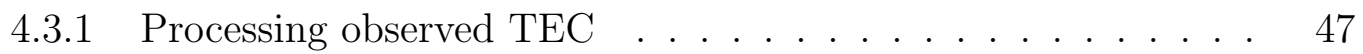

5 TEC OBSERVATIONS AND MODELING OF THE 2016 KAIKOURA EARTH-

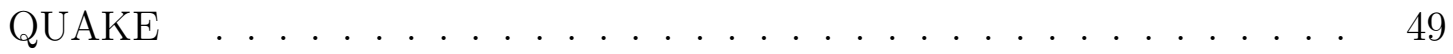

5.1 Abstract . . . . . . . . . . . . . . . 50

5.2 Introduction . . . . . . . . . . . . . . . . . 50

5.3 Geologic Setting and Surface Motion _. . . . . . . . . . 52

5.4 Methods . . . . . . . . . . . . . . . . . . . . . . 53

$5.4 .1 \quad$ IPP locations $\ldots \ldots \ldots \ldots \ldots \ldots \ldots \ldots$

$5.4 .2 \quad$ TEC modeling . . . . . . . . . . . . . . 56

5.4.3 TEC data extraction and processing $\ldots \ldots \ldots \ldots$

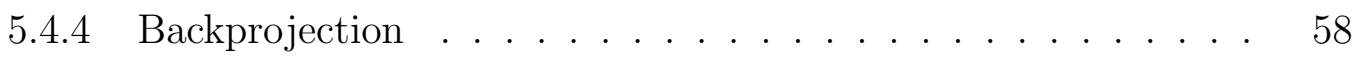

5.5 Results . . . . . . . . . . . . . . . . . . 60

5.5 .1 TID from TEC signal . . . . . . . . . . . . . 60

5.5.2 Backprojection Results . . . . . . . . . . . . . 66

5.6 Discussion . . . . . . . . . . . . . . . . . . . . . . . . . . . . . . 69

5.7 Conclusion . . . . . . . . . . . . . . . . . . . . . . 72

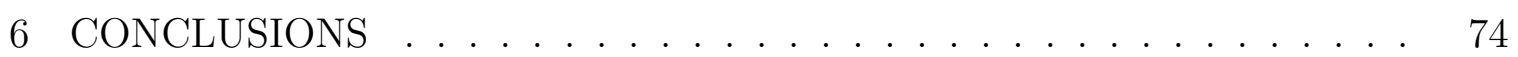

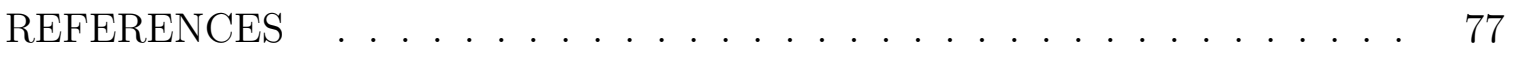

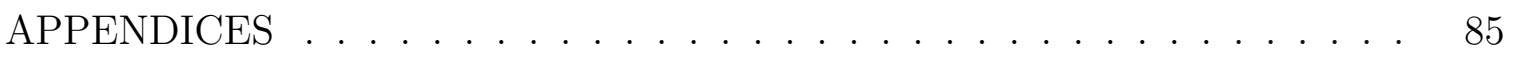


A MISFIT ANALYSIS . . . . . . . . . . . . . . . 85

B VELOCITY ANALYSIS ...................... 89

C ADDITIONAL SENSITIVITY EXAMPLES . . . . . . . . . . . . . 91

D KAIKOURA TEC OBSERVATIONS . . . . . . . . . . . . . . . . 94 


\section{LIST OF FIGURES}

1.1 The IPP is the intersection of the ionospheric height with the satellitereceiver line-of-site $(\mathrm{LOS}) . \ldots \ldots \ldots \ldots$

1.2 Figure from Heki et al. (2006) illustrating the three types of coseismic signals in the ionosphere. The direct acoustic wave is generated by uplift around the epicenter (right side of figure). In the far field (left side of figure) Rayleigh waves generate acoustic waves similar to the first type but travel horizontally at the speed of the Rayleigh waves generating them. Finally, tsunamigenic earthquakes (center of figure) also excite internal gravity waves in the ionosphere. . . . . . . . .

1.3 Comparison of time series plots of vertical electron content (VEC) on the day of the earthquake (right) and on the previous day (left). 1 VEC $=10^{14}$ electrons $/ \mathrm{m}^{2}$. These plots demonstrate signal that is consistent with the 4:31 am earthquake. The blue areas along the IPP tracks between 4.7 and 5.5 am on Jan 17 indicate higher amplitude VEC and are consistent with arrival times from numerical models. Figure from Calais \& Minster (1995). . . . . . . . . . . . . . . . . . 
1.4 Example of typical CID presentations. (a) TEC Map shows the distrubance propagating radially from the area near the epicenter (yellow star). (b) Time series maps of the change to the TEC for azimuths highlighted in insets, plotted in units of TECU $=1 \times 10^{1} 6$ electrons $/ \mathrm{m}^{2}$. Gray solid and dashed lines represent slope of Rayleigh and direct-acoustic waves, respectively. Black rectangle indicates the characteristic N-wave. From Rolland et al. (2011) . . . . . . . . . . .

1.5 Example temperature profile (left) and corresponding velocity profile (right) at ionospheric heights. Profile is for Van, Turkey on October

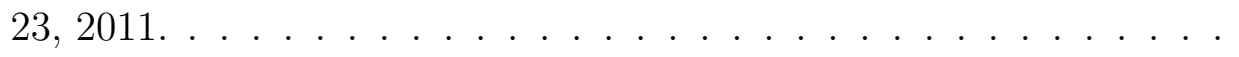

1.6 Literature examples of modeling TEC. (a) Comparison of modeled TEC (colored) with observed (gray) at near field for the 2011 Van, Turkey earthquake. Waveforms for stations in red show the characteristic N-wave. From Rolland et al. (2013). (b) Synthesized TEC from eight point sources for the 2004 Sumatra earthquake. Results for one station paired with two satellites are shown at the top (satellite 23) and bottom (satellite 13). Individual time series for each of the eight sections are at the bottom of each satellite-receiver. The combined synthetic (thick black line) and observed data are plotted directly above the individual time series. Amplitudes are relative as the synthesized signals have arbitrary scaling. From Heki et al. (2006) . . . . . . . . . 
2.1 Illustrations of modeling steps. (a) Example output from raytracing (left) of the travel times of the acoustic wave. Convolving the arrival times with a $\mathrm{N}$-shaped source function produces a time series of the acoustic wave (right) at each point in space. (b) Electron density perturbation after coupling of the acoustic wave with the ionosphere 10 minutes after generation of the acoustic wave. The black line is the line-of-sight from the receiver (triangle) to the satellite. (c) Results from the integration along the line of sight over all times give the TEC time series in TEC units (TECU), where 1 TECU $=10^{16} \mathrm{el} / \mathrm{m}^{2} . \quad$.

2.2 Diagram of the relationship between $\mathbf{L}, \mathbf{K}, \mathbf{r}$, and $\chi$. The red triangle is the ground receiver and $*$ is the source location. . . . . . . .

3.1 Station grid for the Van location. Other locations have grids with similar geometry. . . . . . . . . . . . . . . .

3.2 Examples of the elevation angles for 2 satellites at the $\mathrm{N}$ Mid Lat location. Elevation of angles of zero are when the satellite was below

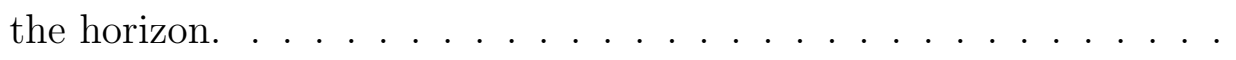

3.3 Waveform comparisons of $1 \mathrm{D}$ and 3D divergence results of modeled TEC. Stations are from the N Mid Lat grid and paired with the same satellite $(\mathrm{G} 21) \ldots \ldots \ldots \ldots \ldots \ldots \ldots$

3.4 Waveform differences and elevation angles for $\mathrm{N}$ Mid Lat grid location. 33

3.5 Waveform differences and elevation angles for N High Lat. . . . . . 35

3.6 Waveform differences and elevation angles for Equator. Note change in the color axis in G21 to show higher errors. . . . . . . . . 36

3.7 Waveform differences and elevation angles for S Mid Lat. . . . . . . 37 
3.8 Waveform differences and elevation angles for S High Lat. . . . . . . . 38

3.9 Global background electron density on October 23, 2011 at 10:50am. 39

4.1 Workflow to extract TEC and convert to a MATLAB binary file. . . . 44

4.2 Example output from plot_compact_2.m. The plot on the left shows all satellites during a 24-hour time period. Satellites below the horizon have no data. The plot on the right displays times immediately before and after the 2011 Van (Turkey) earthquake. The black dashed line indicates the time of the earthquake. . . . . . . . . . .

4.3 Plots of TEC time series for GPS satellite number 21 and station brmn (Turkey). Units are TEC units (TECU) where 1 TECU $=10^{16}$ electrons $/ \mathrm{m}^{2}$. The parabola shape is due to the motion of the satellite as the line-of-sight length through the ionosphere changes as the satellite orbits the Earth. . . . . . . . . . . . . . . .

4.4 Example of processing for a single TEC time series from the 2016 Kaikoura event. The raw data (top), TEC after polynomial detrend (middle), final TEC after filtering (bottom) . . . . . . . .

5.1 Tectonic setting and major faults in New Zealand across the northeast part of the southern island. The large southern star marks the Kaikoura earthquake epicenter estimated by USGS. The gray lines indicate plate boundaries, with other active faults as thin black lines. The star to the northeast of the southern island indicates the second location (Cape Campbell) where significant surface displacements occurred over a wide area (e.g. Hamling et al. (2017)). . . . . . . . . . . . . . . . 
5.2 Location of GNSS stations with IPP tracks for the six satellites for station WGTN (white triangle). Stars for each IPP track correspond to the earthquake onset time. Large star on south island is the initial rupture location. Rectangles are fault planes projected to the surface.

5.3 Left: Atmospheric sound speed profile computed from the NRLMSISE model (Picone et al., 2002) on November 13, 2016 above the rupture area. Center: Atmospheric amplification factor. Right: Horizontal wind model computed from the NRLHWM (Drob et al., 2015) on the same day above Epicenter 1. . . . . . . . . . . . . . . .

5.4 TEC observations from all stations plotted as a function of time and epicentral distance. Distance is calculated from the IPP to two potential epicenters: (left) Epicenter 1 at $42.72^{\circ} \mathrm{S} 173.06^{\circ} \mathrm{E}$ and (middle) Epicenter 2 at $41.73^{\circ} \mathrm{S}, 174.28^{\circ}$. Grayscale (color online) indicates the amplitude of the TEC in TEC units (TECU), where 1 TECU $=1 \times 10^{16}$ electrons per meter squared. The gray line marks the expected arrival time of an acoustic wave traveling radially at $1 \mathrm{~km} / \mathrm{s}$ from the chosen epicenter and the $\mathrm{x}$-intercept corresponds the time for the wave to propagate from the surface to an altitude of $320 \mathrm{~km}$. Right: signalto-noise ratio of observed ( $\mathrm{x}$ and dots) and modeled (squares and triangles) TEC plotted by epicentral distance of the IPP at the time of maximum value of observed TEC for Epicenter 1 and Epicenter 2. . .

5.5 Stations with maximum amplitude $\geq 0.05$ TECU from those in Figure 5.4 for Epicenter 1 (left) and Epicenter 2 (middle). Right: SNR as in Figure 5.4 for selected stations . . . . . . . . . . . . . . . 
5.6 Map of the IPPs selected for the modeling study. Curves show the IPPs trajectories at $320 \mathrm{~km}$ height for LOS linking GNSS stations marked as triangles to satellites G20 (northeast to south east tracks) and R22 (southwest to northeast tracks). The same time window as in Figure 5.2 is used. Additionally, the small stars mark the IPP locations at the earthquake onset time. The disks show the location of IPP when the observed TEC perturbation is maximum. Note that due to the GNSS satellites orbital motion, the IPP trajectories of satellite G20 (respectively R22) here describe a southwestward (respectively southward) translation of the selected network, moving southeastward (respectively northward) with time. The large stars mark the epicenter locations chosen as inputs to models 1 and $2 \ldots \ldots \ldots \ldots$. . . .

5.7 Comparison of observed TEC time series (black, thick line) and modeled TEC using Epicenter 1 (light, thin line) and Epicenter 2 (medium dark line). Time is in reference to the initialization of the Kaikoura rupture and correspondence to time zero for Model 1. Model 2 is shifted 60 seconds to account for the difference in start time. Maximum observed TEC is shown at right, along with the distance from each epicenter to the station and the azimuth of the IPP at the time of maximum TEC. We indicate the maximum TEC time by a black dot for each observed TEC series. Stations are plotted in order of distance to the Epicenter 2 (medium dark line). The scale for the y-axis of each station is shown on the top stations. $\ldots \ldots \ldots \ldots \ldots \ldots$ 
5.8 Backprojection results from TEC synthetic time series for the Epicenter 1 model (large star). Most station and IPP locations lie to the northeast of the epicenter and have varying IPP tracks (e.g. station WGTN in Figure 5.2$) \ldots \ldots \ldots \ldots$. . . . . . . . . . . . 67

5.9 Backprojection results from observed TEC time series. The geographic

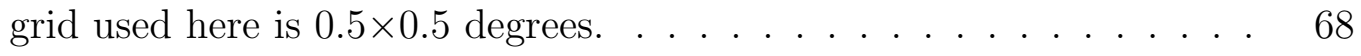

5.10 Averaged backprojection results from satellites G20, R22 and G29 in Figure 5.9. The geographic grid used here is $0.1 \times 0.1$ degrees. . . . . .

A.1 Comparison of 1D and 3D TEC results. (Left panel) Raw waveforms and RMS error for stations near Van, Turkey paired with satellite 21. (Right Panel) Amplitude normalized envelope of the raw waveforms and corresponding RMS error. . . . . . . . . . . . . . 87

A.2 Same as A.1 but for satellite $30 \ldots \ldots \ldots \ldots \ldots$

B.1 Interpolated TEC and best fit line to the maximum amplitude TEC. $\quad 90$

C.1 Waveform differences and elevation angles for satellite G30 at N Mid Lat location. . . . . . . . . . . . . . . . . . 92

C.2 Waveform differences and elevation angles for magnetic Equator. Latitude: $7.58^{\circ}$ Declination: $0.39^{\circ}$, Inclination $0.32^{\circ} \ldots \ldots \ldots$

D.1 TEC time series data for Satellite R22. Stations below the cutoff of 0.05 TECU have been removed. . . . . . . . . . . . . 96

D.2 TEC time series data for Satellite R21. Stations below the cutoff of 0.05 TECU have been removed. . . . . . . . . . . . . . 96 
D.3 TEC time series data for Satellite G29. Stations below the cutoff of 0.05 TECU have been removed. . . . . . . . . . . . . . . . . . 97

D.4 TEC time series data for Satellite G05. Stations below the cutoff of 0.05 TECU have been removed. . . . . . . . . . . . . . . 97

D.5 TEC time series data for Satellite G13. Stations below the cutoff of 0.05 TECU have been removed. . . . . . . . . . . . . . . . . . . 98

D.6 Left: TEC waveform comparison for Satellite 21. Right: Map of IPP locations from the time of the initial rupture to 35 minutes after. Epicenters 1 and 2 are large stars and triangles are the stations on the ground. . . . . . . . . . . . . . . . . .

D.7 Left: TEC waveform comparison for Satellite 29. Right: Map of IPP locations from the time of the initial rupture to 35 minutes after. Epicenters 1 and 2 are large stars and triangles are the stations on the ground.

D.8 Left: TEC waveform comparison for Satellite 05. Right: Map of IPP locations from the time of the initial rupture to 35 minutes after. Epicenters 1 and 2 are large stars and triangles are the stations on the ground. . . . . . . . . . . . . . . . . . . 100

D.9 Left: TEC waveform comparison for Satellite 13. Right: Map of IPP locations from the time of the initial rupture to 35 minutes after. Epicenters 1 and 2 are large stars and triangles are the stations on the ground. 


\section{LIST OF TABLES}

1.1 TID sources and their contribution to TEC. . . . . . . . . . . 4

3.1 Earth's magnetic field at the 4 test locations. Longitude for all loca-

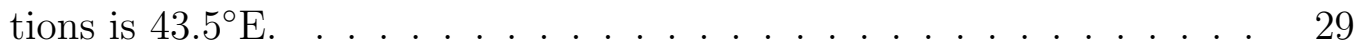

3.2 Times and satellites used to model the TEC at each test location. . . 30 


\section{CHAPTER 1:}

\section{INTRODUCTION \& RESEARCH OBJECTIVE}

\section{$1.1 \quad$ Background}

\subsubsection{The Ionosphere}

The ionosphere impacts modern society in several important ways. Because of the reflective nature of the upper ionosphere, we can send radio and communication transmissions over great distances. Technology must also account for the ionosphere as disturbances in the ionosphere can interrupt signals from satellites. At high latitudes, the auroras create currents that can reach up to a million amperes and induce currents in power lines and pipelines (University of Alaska Fairbanks Geophysical Institute, 2017). The ionosphere also contains information of what happens in the Earth's geospheres, and therefore is a means of remotely sensing processes occurring at the surface of the hydrosphere, the lithosphere and the atmosphere or ionosphere itself. Additionally, the ionosphere is the closest naturally occurring plasma, the most common form of matter in the universe. Studying the ionosphere can help us understand this phase of matter.

The ionosphere is a region of the Earth's atmosphere where the sun's radiation has stripped neutral atoms of one or more electrons, thus producing free electrons. The ionosphere consists of several distinct layers (Schunk \& Nagy, 2000). The D 
region exists only during daylight and extends from about $50 \mathrm{~km}$ to $90 \mathrm{~km}$ and has low electron densities. The E region extends from $90-150 \mathrm{~km}$ and is more diffuse at night. Finally, the F region is divided into two layers, the F1 and F2 regions. During the night, the F1 layer decays creating a separation between the E and F2 layer. The majority of the electron density in the ionosphere falls within the F2 layer at about $300 \mathrm{~km}$.

Disturbances to the ionosphere naturally occur at many different wavelengths from planetary to local scales. On a planetary level, Rossby waves result from variations with latitude of the strength of the Coriolis effect and have wavelengths thousands of kilometers long (Beer, 1974). At the medium and large scale, acoustic gravity waves propagate at tens to hundreds of meters per second with wavelengths of 100$300 \mathrm{~km}$ for the medium scale and 300-3000 km for the large scale (Schunk \& Nagy, 2000). Collectively, any disturbance propagating through the ionosphere is known as a traveling ionospheric disturbance (TID).

\subsubsection{Total Electron Content}

TIDs are common and have a variety of sources in both the solid-Earth as well as the atmosphere, including volcanic eruptions, earthquakes, tsunamis, large storms, solar flares, meteorite impacts and human-caused sources such as explosions and rocket launches (Pichon et al., 2009). When energy from a solid-Earth based source couples into the atmosphere it creates a propagating acoustic wave. The acoustic wave travels through the atmosphere and couples with the ionosphere to produce perturbations in the electron density. These perturbations disrupt signals from global navigation space systems (GNSS), for example the U.S. global positioning system (GPS), the Russian global navigation satellite system (GLONASS) or the European satellite constellation, 
Galileo.

The interruption to GNSS by ionospheric disturbances is a result of the cumulative effects of the total electron content (TEC) along the satellite-receiver line of sight (LOS). GNSS automatically corrects these disruptions to the satellite signal by transmitting two frequencies. From these frequencies, the GNSS accounts for the TEC and returns travel times from the satellite to the receiver. Calais \& Minster (1995) outline the method to obtain the TEC from GNSS data. The University Navstar Consortium (UNAVCO) also provides a software to obtain the TEC which is available online and which I use for this thesis (see Chapter 4). UNAVCO is a non-profit universitygoverned consortium that is funded through the National Science Foundation (NSF) and NASA.

Table 1.1 summarizes the impact of some common sources of TIDs on the TEC. The TEC perturbations are given either in TEC units (TECU), where 1 TECU = $10^{16}$ electrons $/ \mathrm{m}^{2}$, or in percent change relative to the absolute TECU.

\subsubsection{Ionospheric Pierce Point}

It is important to note that the TEC is obtained from summation along the entire line of sight. Here an assumption is generally made in order to assign the TEC measurement to a single location in the ionosphere. We first assume that the majority of the electron content resides within a narrow range of ionospheric heights and then define a height for an infinitely thin ionosphere. The height of this ionospheric layer is most often the height of the maximum electron density, but not exclusively. The intersection of this ionospheric height with the line of sight is used as the point of origin for the TEC measurement and is called the ionospheric pierce point (IPP). Figure 1.1 gives an illustration of the IPP. In this case, the IPP samples a region of 
Table 1.1: TID sources and their contribution to TEC.

\begin{tabular}{|c|c|c|c|}
\hline Source & TEC Perturbation & $\begin{array}{c}\text { Period } \\
(\mathrm{min})\end{array}$ & Reference \\
\hline solar eclipses & $0.15-15$ TECU & - & Afraimovich et al. $(2013)$ \\
\hline solar terminator & $0.2-1$ TECU & 15 or 60 & Afraimovich et al. $(2013)$ \\
\hline solar flare & $\sim 0.5$ TECU & $20-60$ & Afraimovich et al. $(2013)$ \\
\hline tropical cyclone & 2.5 TECU & $1-150$ & Afraimovich et al. $(2013)$ \\
\hline rocket launches & $0.2-2 \%$ & $3-8$ & Afraimovich et al. $(2013)$ \\
\hline geomagnetic storms & $10-14 \%$ & - & Afraimovich et al. $(2013)$ \\
\hline earthquakes & $1.8-6 \%$ & $4-5$ & $\begin{array}{c}\text { Astafyeva } \text { et al. }(2014) \\
\text { Heki } \text { et al. }(2006)\end{array}$ \\
\hline tsunami & 0.15 & $8.7-14$ & $\begin{array}{c}\text { Grawe \& Makela }(2015) \\
\text { Occhipinti } \text { et al. }(2013)\end{array}$ \\
\hline
\end{tabular}

* TEC perturbation is at peak intensity. The effect disappears once winds are below $30 \mathrm{~m} / \mathrm{s}$.

the ionosphere off the coast, which demonstrates the utility of the TEC to sample over sources of subduction zone earthquakes in the ocean.

\section{$1.2 \quad$ Identifying CIDs}

A significant amount of research has demonstrated that coseismic ionospheric disturbances (CIDs) can be identified in TEC signals obtained by GNSS (Calais \& Minster, 1995; Artru et al., 2005; Afraimovich et al., 2010; Occhipinti et al., 2013; Astafyeva et al., 2014). The literature identifies CIDs in two primary ways. Here I briefly describe these two methods and then show examples in the following section.

First, time series plots of receiver arrays and paired satellites show perturbations with velocities consistent with three different signals produced by earthquakes and tsunamis (Heki et al., 2006; Occhipinti et al., 2013). Heki et al. (2006) give an illustration of these 3 signals that is reproduced here in Figure 1.2. Each class of 


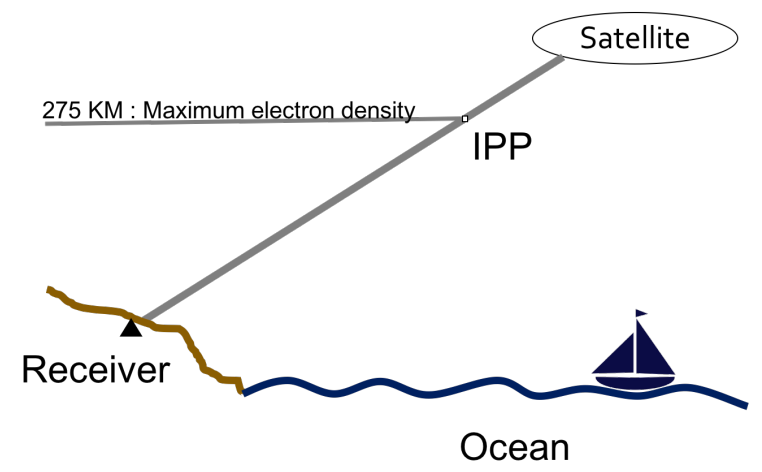

Figure 1.1: The IPP is the intersection of the ionospheric height with the satellite-receiver line-of-site (LOS).

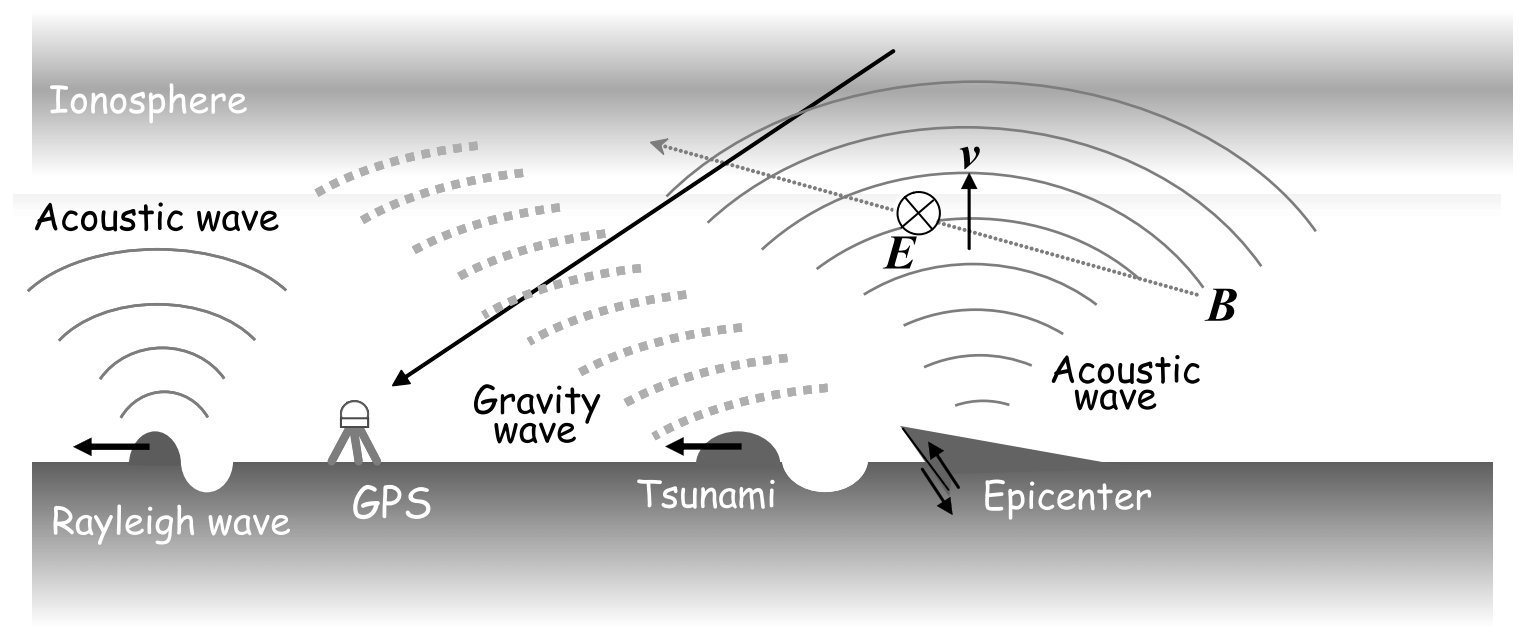

Figure 1.2: Figure from Heki et al. (2006) illustrating the three types of coseismic signals in the ionosphere. The direct acoustic wave is generated by uplift around the epicenter (right side of figure). In the far field (left side of figure) Rayleigh waves generate acoustic waves similar to the first type but travel horizontally at the speed of the Rayleigh waves generating them. Finally, tsunamigenic earthquakes (center of figure) also excite internal gravity waves in the ionosphere. 

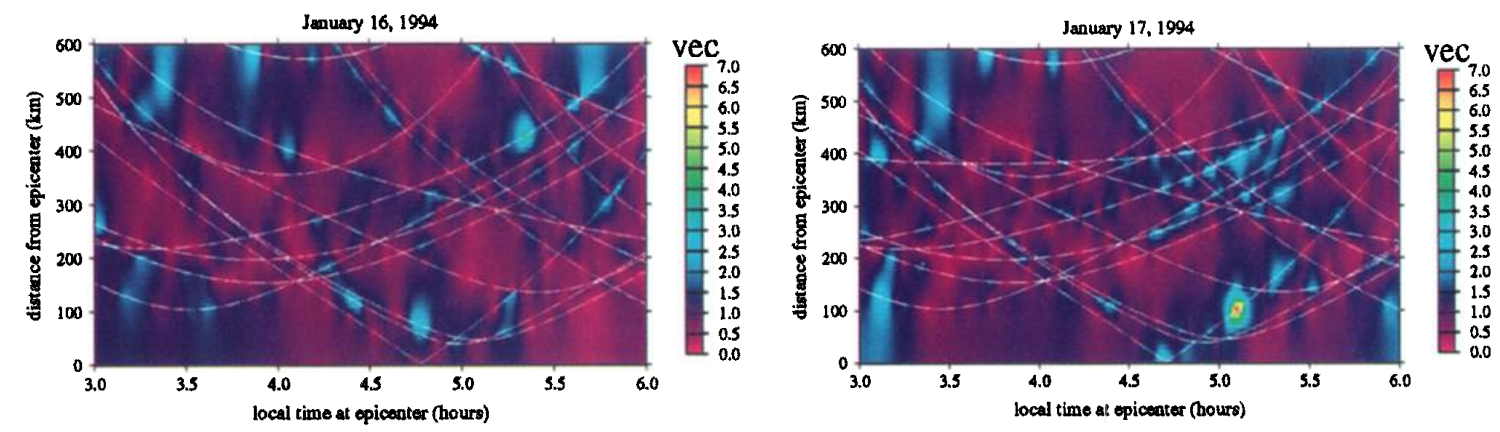

Figure 1.3: Comparison of time series plots of vertical electron content (VEC) on the day of the earthquake (right) and on the previous day (left). $1 \mathrm{VEC}=10^{14}$ electrons $/ \mathbf{m}^{2}$. These plots demonstrate signal that is consistent with the 4:31 am earthquake. The blue areas along the IPP tracks between 4.7 and 5.5 am on Jan 17 indicate higher amplitude VEC and are consistent with arrival times from numerical models. Figure from Calais \& Minster (1995).

signal is labeled in bold font and includes: (1) the direct acoustic wave from the uplift at the source (Heki \& Ping, 2005; Afraimovich et al., 2010; Rolland et al., 2013; Occhipinti et al., 2013), (2) perturbations caused from Rayleigh waves traveling on the surface (Rolland et al., 2011; Occhipinti et al., 2013) and (3) internal gravity waves created by tsunamigenic earthquakes (Heki \& Ping, 2005; Occhipinti et al., 2013). My research focuses on the direct acoustic wave signal. I discuss this class of CID for the remainder of this thesis. The second way researchers have confirmed the identity of CIDs is with numerical modeling of TEC response to CIDs (Heki et al., 2006; Rolland et al., 2011; Kherani et al., 2012; Rolland et al., 2013), which has shown good agreement with observations. In the following sections I give some examples of identifying CIDs from the literature.

\subsubsection{TEC Time Series}

Calais \& Minster (1995) were the first to observe CIDs using the GNSS-TEC tech- 
nique. They observe fluctuations in electron content up to $1000 \mathrm{~km}$ (along the ground) from the January 17 1994, $\mathrm{M}_{w} 6.7$ Northridge (CA) earthquake. Figure 1.3 (right) shows a 3 hour time series window for January 16th and 17th. The IPP tracks are plotted as white dots and, through time, show a parabolic shape due to the motion of the satellite. The authors use the vertical electron content (VEC), which is the electron content summed vertically from a point in the ionosphere to the ground. The VEC is obtained from the TEC using basic trigonometry as VEC $=\frac{h}{L} \mathrm{TEC}$, where $\mathrm{h}$ is the thickness of the ionosphere and $\mathrm{L}$ is the length of the ray path through the ionosphere. The January 17 plot (right) shows VEC with $1 \%-3 \%$ change in total electron content between the hours of 4:40 and 5:30 am local time at distances of about $100 \mathrm{~km}$ as well as between $220-450 \mathrm{~km}$, which they note is consistent with previous observations after moderate-size earthquakes or nuclear explosions. Numerical simulations (Davies \& Archambeau, 1996; Warshaw \& Dubois, 1981) predict that the direct acoustic wave would arrive at ionospheric heights 10 to 15 minutes after ground displacement. The arrival times of the perturbations are therefore consistent with numerical simulations for the 4:31am (local time) earthquake. Figure 1.3 also shows the VEC for the previous day (left). No similar organized perturbation exists, further supporting the conclusion that the higher amplitudes in the VEC were created by an earthquake on the surface. The authors also compute the velocities of the first arrivals as 300 to $600 \mathrm{~m} / \mathrm{s}$. Because the velocity is in good agreement with numerical models of ionospheric acoustic-gravity waves generated by seismic sources, they interpret the source as the acoustic wave generated by uplift in the epicentral region.

Since the Calais \& Minster (1995) paper, identifying CIDs through TEC is rou- 

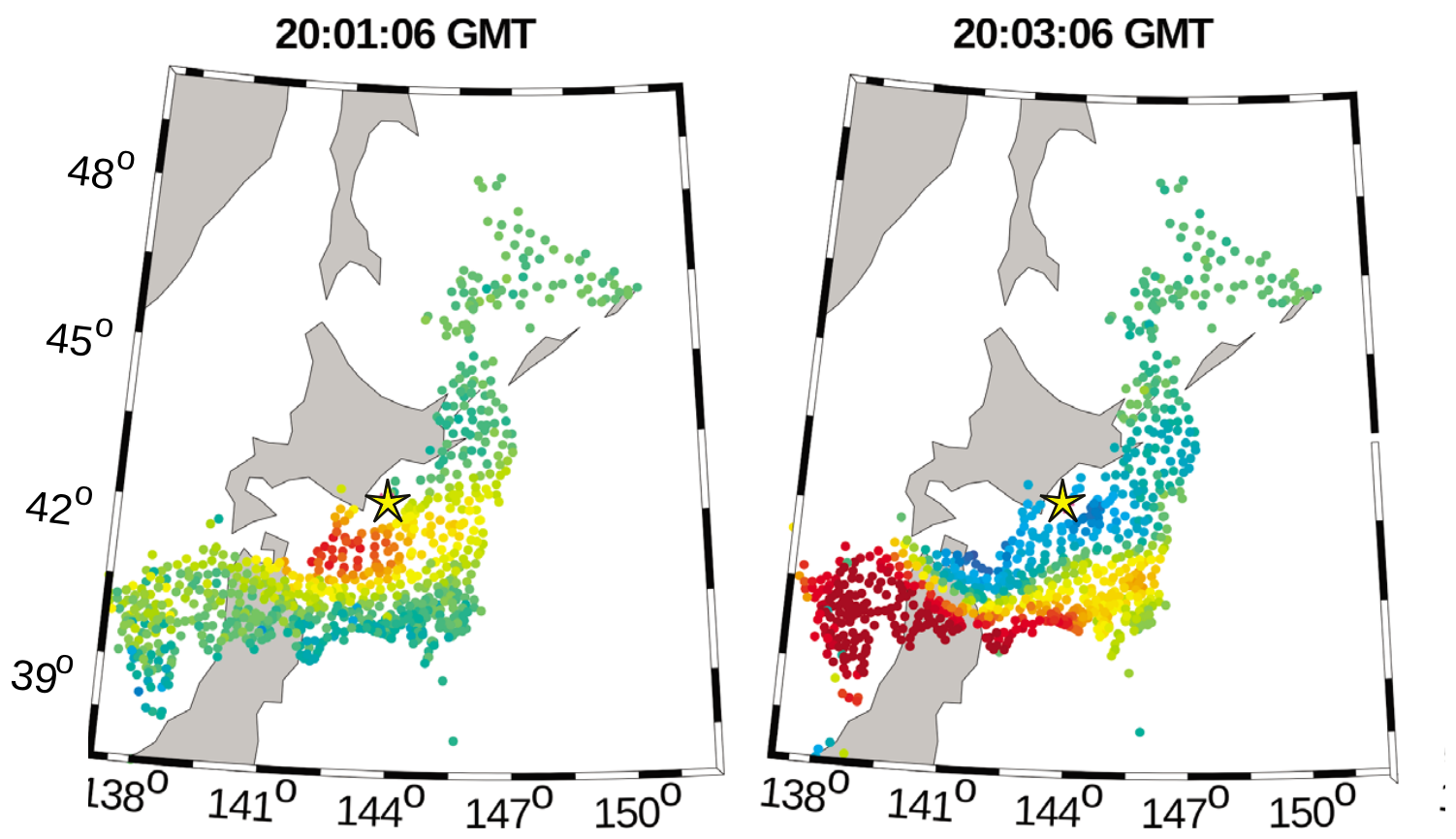

(a)
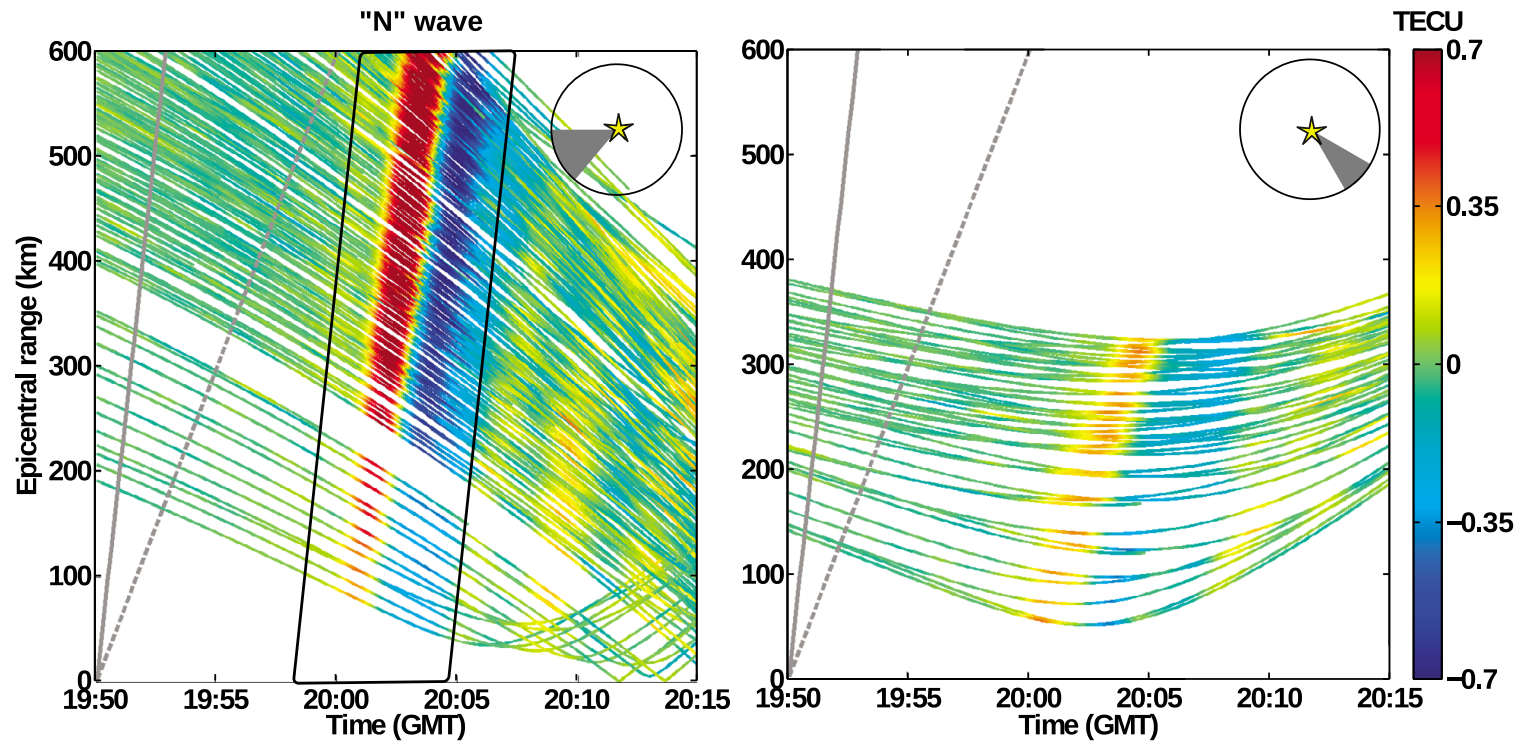

(b)

Figure 1.4: Example of typical CID presentations. (a) TEC Map shows the distrubance propagating radially from the area near the epicenter (yellow star). (b) Time series maps of the change to the TEC for azimuths highlighted in insets, plotted in units of TECU $=1 \times 10^{1} 6$ electrons $/ \mathbf{m}^{2}$. Gray solid and dashed lines represent slope of Rayleigh and direct-acoustic waves, respectively. Black rectangle indicates the characteristic N-wave. From Rolland et al. (2011) 
tinely demonstrated through TEC maps in which the authors show the projection of the IPPs on the ground with a color map of the TEC amplitude, as well as through time series plots (see for example Heki \& Ping (2005); Kherani et al. (2012); Occhipinti et al. (2013); Grawe \& Makela (2015); Gõmez et al. (2015)). Often the TEC is plotted as the change in the TEC by removing a polynomial fit of the TEC. This removes the influence of the moving satellite on the TEC.

An example from Rolland et al. (2011) (Figure 1.4) shows both types of plots for the Tokachi-Oki earthquake. The origin time is 19:50 UTC. Figure 1.4a shows two TEC maps at 11 and 13 minutes after the origin time. Figure 1.4b shows two TEC time series for different azimuthal coverages (shown in circular insets). The gray lines in Figure 1.4b show the slope of Rayleigh (solid) and direct acoustic (dashed) waves. The lines do not account for the time to propagate to ionosphere and so there is an apparent delay in the arrival of the CID. Both the TEC maps and the time series show a very clear signal. The TEC map shows a disturbance radiating from around the area of the epicenter (marked by a yellow star). All of these plots provide visual evidence of a CID created by an earthquake source. Additionally, the velocities of these CIDs are consistent with CID generated by direct-acoustic and Rayleigh waves. In the west, the CID follows the slope of the Rayleigh wave whereas in the East the slope of the CID is consistent with sound speed and therefore the direct acoustic wave. This is due to the radiation pattern of the Rayleigh wave. The IPPs to the southwest sample the much greater amplitude of the Rayleigh wave contribution. 

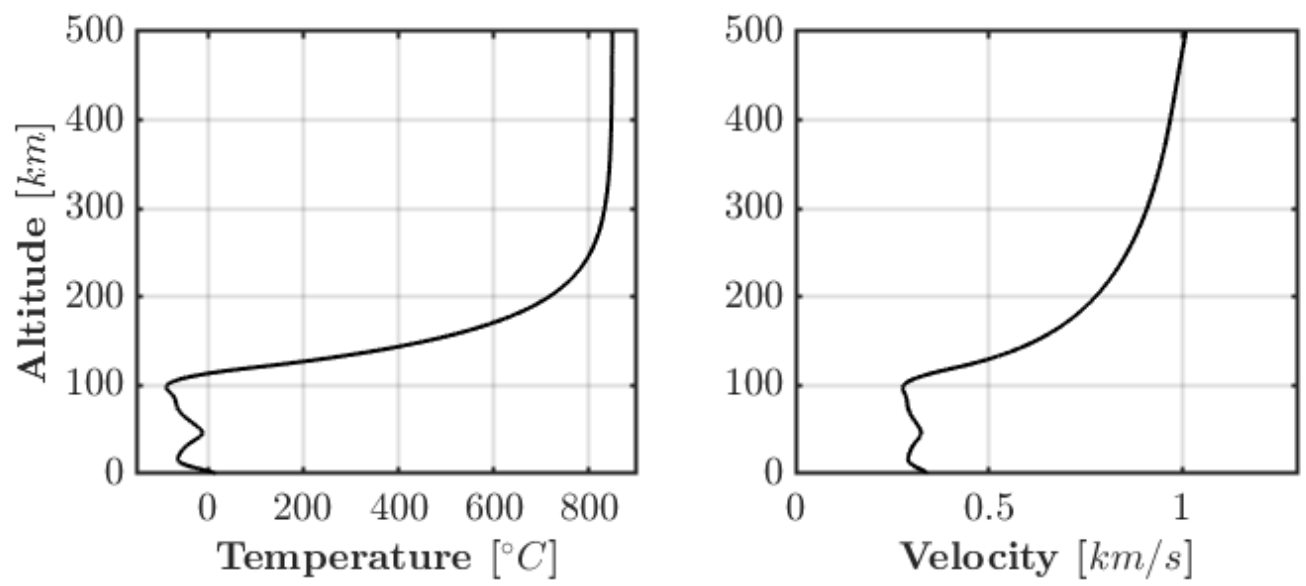

Figure 1.5: Example temperature profile (left) and corresponding velocity profile (right) at ionospheric heights. Profile is for Van, Turkey on October 23, 2011.

\section{Sound Speed}

For perfect gases, sound travels at a velocity given by:

$$
v^{2}=\gamma p / \rho=\gamma R T
$$

where $\gamma$ is the ratio of specific heats $\left(\frac{c_{p}}{c_{v}}\right)$, p is pressure, $\rho$ is the density of the air, $\mathrm{R}$ is the gas constant and $\mathrm{T}$ is the temperature $(\mathrm{K})$. Therefore, the speed of sound is most influenced by temperature. Figure 1.5 shows an example profile of the temperature and resulting sound speed at different atmospheric heights. At the ionospheric height of $300 \mathrm{~km}$, the temperature is $832^{\circ}$ and the speed of sound is $900 \mathrm{~m} / \mathrm{s}$.

When the velocity of air particles becomes close to the speed of sound a "shockacoustic" effect occurs (Afraimovich et al., 2001) due to non-linear effects (Chum et al., 2016). Large earthquakes produce acoustic waves that approach the speed of sound and thus the original waveform is transformed into an N-shaped wave. Figure 
1.4b shows an example of such a wave outlined by the rectangle and is also shown for individual waveforms in Figure 1.6a in red.

\subsubsection{Modeling}

A second method to support identification of a CID is to compare modeled output of a CID to observed data. Kherani et al. (2012) use finite differences to model tsunami-triggered acoustic gravity waves (AGWs) in the atmosphere as well as the ionospheric response. They find good agreement with observed data for the 2011 Tohoku-Oki tsunami.

Another method replaces finite difference calculations with acoustic ray tracing to model the acoustic wave, and is computationally less expensive (Heki \& Ping, 2005; Dautermann et al., 2009; Rolland et al., 2013) . This method still employs finite differences in the propagation of the acoustic wave in the atmosphere and coupling with the ionosphere. This is the model I employ in this study. I describe the methods for the model in Chapter 2. Figure 1.6 shows two examples of this type of modeling from Rolland et al. (2011) and Heki et al. (2006) using single (1.6a) and multiple (1.6b) point sources, respectively.

Rolland et al. (2013) successfully model the TEC response to acoustic waves generated by seismic sources. Figure 1.6a shows their model (colored), which agrees well with observed phase of the data (gray) from the $2011 \mathrm{M} \omega$ 7.1 Van earthquake.

Heki et al. (2006) synthesize TEC in fair agreement with observed data considering multiple source locations along the area ruptured during the 2004 Sumatra earthquake. The authors calculate synthetic CIDs for eight point sources and allow them to interfere with each other to generate the TEC. They successfully predict the the shape of the first peak in TEC but with a time delay (see Figure 1.6b). However, 


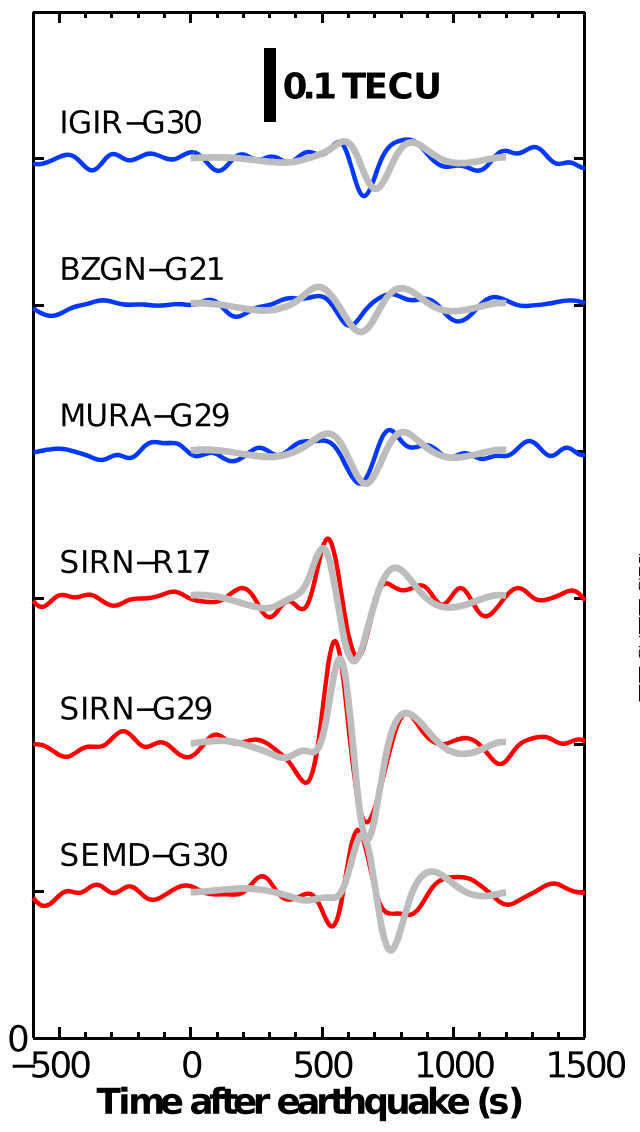

(a)

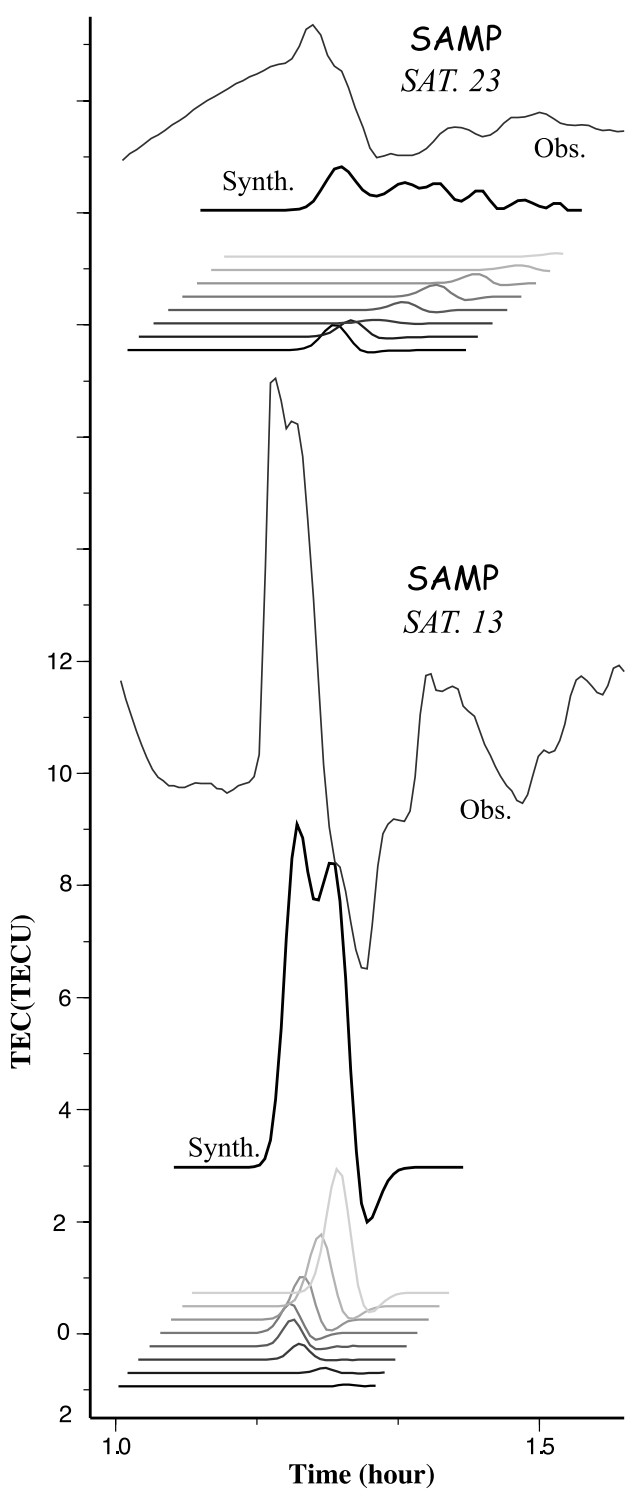

(b)

Figure 1.6: Literature examples of modeling TEC. (a) Comparison of modeled TEC (colored) with observed (gray) at near field for the 2011 Van, Turkey earthquake. Waveforms for stations in red show the characteristic N-wave. From Rolland et al. (2013). (b) Synthesized TEC from eight point sources for the 2004 Sumatra earthquake. Results for one station paired with two satellites are shown at the top (satellite 23) and bottom (satellite 13). Individual time series for each of the eight sections are at the bottom of each satellite-receiver. The combined synthetic (thick black line) and observed data are plotted directly above the individual time series. Amplitudes are relative as the synthesized signals have arbitrary scaling. From Heki et al. (2006) 
their model requires the use of a-posteriori information in order to assign relative amplitudes to the eight acoustic waves generated at each segment. This second example of modeling TEC demonstrates the potential to recover source information from the TEC, specifically, the epicentral location on the surface as well as extent of rupture.

\subsection{Source Information in TEC}

Similar to the Heki et al. (2006) example, other research has begun to investigate the potential of TEC to recover earthquake source information. Some of the source information recovered includes earthquake location (Heki et al., 2006; Liu et al., 2010), lateral rupture extent (Heki et al., 2006), and slip mode (Rolland et al., 2013). Astafyeva \& Heki (2009) suggest that the initial polarity of the TEC might be related to the initial motion of the coseismic neutral pressure wave. Rolland et al. (2013) test this hypothesis and demonstrate that the polarity is primarily linked to local geomagnetic field. It is worth noting however, in places where the geomagnetic field is parallel to the neutral disturbance, the initial polarity can reflect the direction of the first motion on the ground.

Liu et al. (2010) also locate the source of coseismic ionospheric disturbances for the September 20, $1999 \mathrm{M} \omega 7.6$ earthquake using ray tracing and beam-forming techniques. For a given 1D velocity model, they guess the location of a hypo-center and use ray-tracing to calculate the arrival time to each seismometer. From the difference between the calculated and observed arrival time, they select a new location and iterate. The iteration stops when the differences reach a minimum. They also use beam-forming to guess a hypocenter and compute the speeds and associated standard deviation by dividing the distances between the hypocenter and the seismometers by the differences in calculated and observed arrival times. When the standard deviation 
reaches a minimum that location is chosen as the hypocenter.

Finally, Rolland et al. (2013) show that the slip mode can also be inferred from TEC. They use a compressional point source consistent with a reverse fault rupture to model the TEC response to the 2011 Van earthquake (Turkey). The modeled data is in good agreement with observed data.

\subsection{Significance}

Because of the source information contained in the TEC, GNSS-TEC measurements play a potentially important role in earthquake and tsunami monitoring. The acoustic wave, generated by earthquake displacement on the surface, travels to ionospheric heights within about 10 minutes. This creates the potential for near real-time characterization of surface uplift around the epicenter, whether on land or under the ocean, as the displacement on the ocean floor transfers directly to the water (Occhipinti et al., 2013). While seismology offers useful tools to characterize the source, it is limited by the proximity of seismic stations to the sources. TEC measurements offer a method to obtain data closer to the source, due to the ability to image the ionosphere along a line of sight that passes over water (see Figure 1.1). Moreover, the wavefield that propagates in the atmosphere is much less distorted than the wavefield that propagates in the Earth. This makes interpreting waveforms for source characteristics much simpler.

\subsection{Research Objective}

The objective of this thesis is to apply seismic methods to CID created by earthquakes and/or tsunamis. GNSS provide measurements of CID with TEC data, which can be processed to supplement seismic data. Because the TEC is itself a waveform, this 
allows the application of tools from seismology to characterize the source phenomena. However, before we can successfully apply seismic techniques, we must take into account the physics of the CIDs traveling through the ionosphere. I will go into more detail on the physics of the wave propagation in the next chapter, but will highlight the main points here.

CIDs are created initially from acoustic (pressure) waves in the neutral atmosphere generated by vertical motion on the surface. The neutral atmospheric waves couple with the ionosphere in a complex way depending on the local geomagnetic field. There is also a change in the amplitude of the TEC response that depends on the geometry of the satellite and receiver. The situation is further complicated by the satellites' motion. All of these factors need to be taken into account if we are to use the TEC to obtain source characteristics.

One method to do this is to invert for the acoustic wave and was first suggested by Gõmez et al. (2015). The TEC is equal to the acoustic wave times a transfer function in the frequency domain. To invert for the acoustic wave, they divide the observed TEC by the transfer function. To obtain the transfer function, they rely on an analytical model developed by Georges \& Hooke (1970) (hereafter G\&H analytical method). This method relies on two key assumptions: 1) a plane wave approximation of the propagating wave and 2) a 1D electron density divergence in the vertical direction that is approximated by an analytical model of the ionosphere. They successfully apply this method to far-field (Antarctica) TEC signal produced by Rayleigh waves from the 2010 Maule and 2011 Tohoku-Oki earthquakes.

Obtaining the transfer function removes the phase differences between the acoustic wave and the TEC. This will result in more successful application of seismic methods 
to the CID. The first part of this thesis investigates the applicability of the $\mathrm{G} \& \mathrm{H}$ analytical method to CID created by direct uplift around the epicenter. I examine one of the key assumptions that the electron density only varies significantly in the vertical direction. My research question for this part is: Under what (if any) conditions can we reliably assume a 1D ionosphere? I hypothesize that latitude as well as elevation angle of the satellite and geometry of the satellite-receiver line-of-sight may interact to create conditions that determine the applicability of the 1D ionosphere assumption. I expect that at lower elevation angles, the difference between a 1D and $3 \mathrm{D}$ ionosphere is greater due to the increased horizontal component of the line-ofsight.

To test this hypothesis, I use the spherical numerical model outlined by Rolland et al. (2013) to examine the importance of a 3D ionosphere to the TEC. I describe both this numerical model and the G\&H analytical method in the next chapter. I use the spherical numerical method to model the TEC using a 1D and 3D ionosphere at different latitudes and present the results in Chapter 3.

For the second part of my thesis, I examine observed data and illustrate the insights that TEC can provide related to the acoustic source from large earthquakes, as well as an example of how seismic methods can be applied to the CID data. First, I provide background in Chapter 4 of the extraction process of the TEC from GNSS travel times. Chapter 5 is a paper that my coauthors and I submitted to the Bulletin of the Seismological Society of America (BSSA) at the end of September, 2017. We show the strong CID response as measured by the TEC and show that arrival times are consistent with a source on the northeast end of the rupture area. This demonstrates some of the insights that TEC provides. By modeling TEC from different point- 
source locations, we determine which rupture area produced the largest contribution to the ionosphere response. We then apply backprojection to the TEC for the first time, demonstrating the potential of using seismic methods with CID. 


\section{CHAPTER 2:}

\section{FORWARD MODELING TEC}

\subsection{Chapter Summary}

I use the model outlined by Rolland et al. (2013) to investigate the influence of the 3D ionosphere on the TEC, and therefore the assumption of Georges \& Hooke (1970) of a horizontally invarient ionosphere. In this chapter, I summarize the derivations of the TEC equations for both models in separate sections. Within each section, I describe the derivations in terms of three steps. These steps are illustrated in Figure 2.1 and described in greater detail in subsequent sections. They include: (1) generation and propagation of the acoustic wave, (2) electron density perturbations excited by the coupling of the acoustic wave with the ionosphere, and (3) integration of electron density perturbations along the line of site of the GNSS satellite-receiver pair.

\section{$2.2 \quad$ Numerical Method}

\subsubsection{Acoustic Wave}

Figure 2.1a gives an illustration of the first step in the forward modeling of the TEC in which I model the generation and propagation of the neutral acoustic wave. Distance is relative to the acoustic source. I use a ray tracing program to calculate the arrival

time, $t$, wave vector, $\mathbf{k}$, and ray amplitude for a point-like compressional source (Dessa 

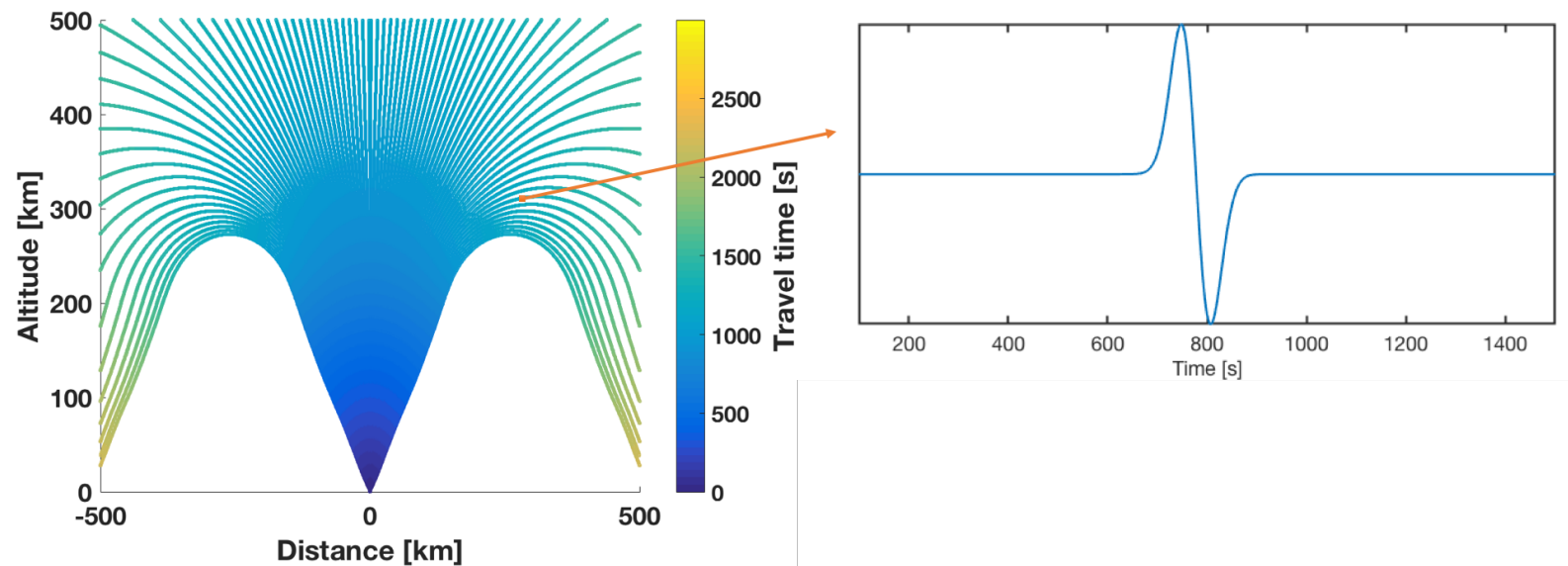

(a)

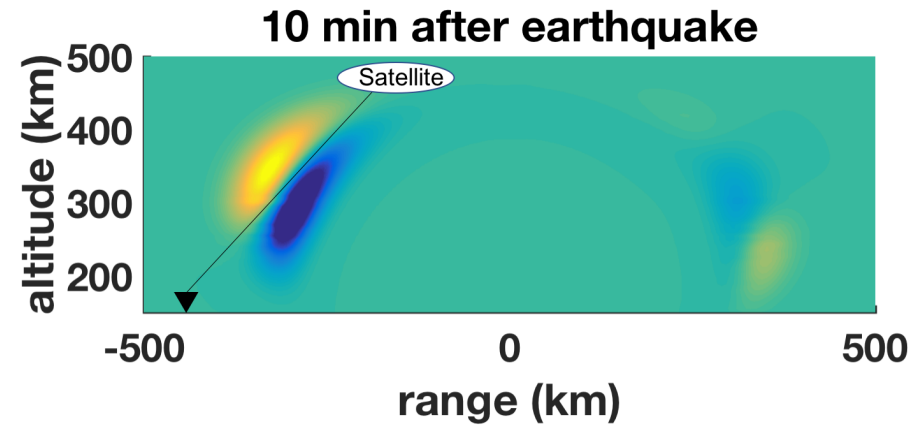

(b)

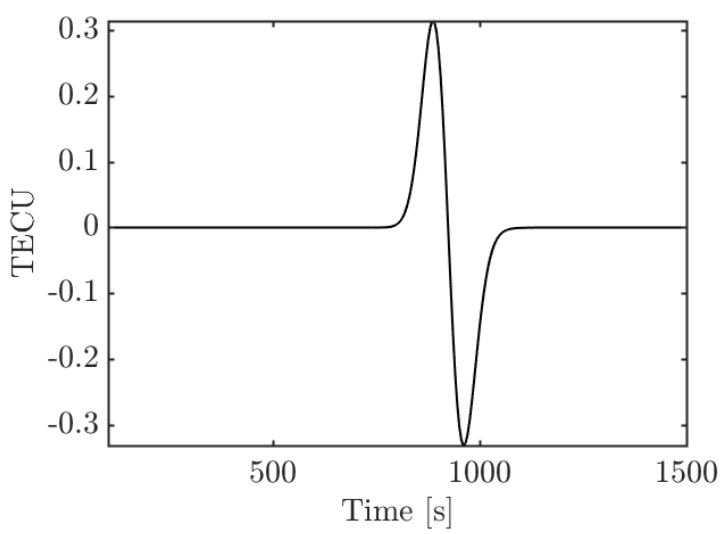

(c)

Figure 2.1: Illustrations of modeling steps. (a) Example output from raytracing (left) of the travel times of the acoustic wave. Convolving the arrival times with a $\mathrm{N}$-shaped source function produces a time series of the acoustic wave (right) at each point in space. (b) Electron density perturbation after coupling of the acoustic wave with the ionosphere 10 minutes after generation of the acoustic wave. The black line is the lineof-sight from the receiver (triangle) to the satellite. (c) Results from the integration along the line of sight over all times give the TEC time series in TEC units (TECU), where 1 TECU $=10^{16} \mathrm{el} / \mathrm{m}^{2}$. 
et al., 2005). An example of the output of the arrival times is shown on the left in Figure 2.1a. Note that each ray follows a curved path that is due to refraction of the acoustic wave by the atmosphere. Next, we convolve the ray amplitude with an N-wave source function (Heki \& Ping, 2005; Dautermann et al., 2009) characteristic of the nonlinear effects of the propagation of the acoustic wave (see 1.2.1 or Chum et al. (2016) for further explanation). This source function describes the atmospheric response to the piston-like motion of the ground at ground level:

$$
u(t)=\frac{A \sqrt{2}}{\sigma^{3 / 2} \pi^{1 / 4}}\left(t-t_{0}\right) e^{-} \frac{\left(t-t_{0}\right)^{2}}{2 \sigma^{2}},
$$

where $\mathrm{A}$ is the initial amplitude factor, $t_{0}$ is the time of maximum displacement and $\sigma$ is the width of the pulse (Rolland et al., 2013). The model also accounts for the frequency-dependent viscous and thermal losses by scaling the pulse width so that it varies linearly as $\sigma(t, \mathbf{r})=b t_{\text {arrival }}(\mathbf{r})$, where $\mathrm{t}_{\text {arrival }}$ is the arrival time, and $\mathrm{b}$ is a scaling factor. The neutral wave particle velocity is then:

$$
\mathbf{v}_{n}=u \mathbf{k}
$$

The result of the convolution produces a time series at all points in space. I show an example result on the right in Figure 2.1a.

\subsubsection{Ionospheric Coupling}

The second step in the forward modeling of the TEC is to find the change in the background electron density $\left(\partial n_{e}\right)$ through time. An example of the results for this step is shown in Figure 2.1b. I describe the physics and derivation of the governing equation in what follows. 
The neutral acoustic wave transfers its momentum directly to charged particles in the ionosphere. The velocity of the charged ions, $\mathbf{v}_{\mathbf{i}}$, is equal to the component of the neutral wave parallel with the geomagnetic field (Georges \& Hooke, 1970):

$$
\mathbf{v}_{\mathbf{i}}=\left(\mathbf{v}_{\mathbf{n}} \cdot \hat{\mathbf{b}}\right) \hat{\mathbf{b}}=\left|\mathbf{v}_{\mathbf{n}}\right||\mathbf{b}| \cos \theta
$$

where $\mathbf{v}_{\mathbf{n}}$ is the particle velocity of the neutral wave and $\hat{\mathbf{b}}$ is the geomagnetic field direction. Equation (2.3) is commonly referred to as the ionospheric coupling factor (hereafter coupling factor), and is a fundamental description of atmosphereionosphere coupling.

I want to find the change in the electron density, $\partial n_{e}$ and so I use the continuity equation as the governing equation:

$$
\frac{\partial n_{e}}{\partial t}+\nabla \cdot\left(n_{e 0} \mathbf{v}_{\mathbf{i}}\right)=0
$$

where $n_{e 0}$ is the unperturbed electron density. In the remainder of this section I examine the 3D and 1D numerical solution to Equation 2.4 using a spherical wavefront. I address the analytical solution in Section 2.3.2. The analytical solution assumes a planar wave.

\section{D Divergence}

Rolland et al. (2011) derive the 3D electron density, $\partial n e$, by solving the governing equation (Equation 2.4) using finite differences and integrating over time:

$$
\partial n_{e}(\mathbf{r}, t)=-\int_{0}^{t} \nabla \cdot\left(n_{e 0} \mathbf{v}_{\mathbf{i}}(\mathbf{r}, t)\right) d t
$$


If we expand the integrand as:

$$
\nabla \cdot\left(n_{e 0}\left(\mathbf{v}_{\mathbf{i}}\right)\right)=n_{e 0}\left(\nabla \cdot \mathbf{v}_{\mathbf{i}}\right)+\mathbf{v}_{\mathbf{i}} \cdot \nabla n_{e 0}
$$

we see that the perturbations in the electron density depend on both the divergence of the ionospheric wave and the gradient of the background electron density.

In spherical coordinates, Equation (2.6) is

$$
\begin{array}{r}
\nabla \cdot\left(n_{e 0}\left(\mathbf{v}_{\mathbf{i}}\right)\right)=n_{e 0}\left[\left(\frac{2}{r} v_{i r}+\frac{\partial v_{i r}}{\partial r}\right)+\left(\frac{1}{r} \frac{\partial v_{i \theta}}{\partial \theta}+\frac{v_{i \theta} \cos \theta}{r \sin \theta}\right)+\left(\frac{1}{r \sin \theta} \frac{\partial v_{i \phi}}{\partial \phi}\right)\right] \\
+v_{i r} \frac{\partial \eta_{e 0}}{\partial r}+\frac{v_{i \theta}}{r} \frac{\partial \eta_{e 0}}{\partial \theta}+\frac{v_{i \phi}}{r \sin \theta} \frac{\partial \eta_{e 0}}{\partial \phi} .
\end{array}
$$

This 3D model serves as the baseline for analysis on this project. In order to analyze the applicability of the analytical model used by Gõmez et al. (2015), I also run the model using a simplified 1D divergence that is comparable to the analytic model.

\section{D Divergence}

We can simplify Equation 2.7 to one dimension if we assume that only the partial derivative in the $\mathrm{r}$ direction is significant:

$$
\nabla \cdot\left(n_{e 0}\left(\mathbf{v}_{\mathbf{i}}\right)\right)=n_{e 0}\left[\frac{2}{r} v_{i r}+\frac{\partial v_{i r}}{\partial r}\right]+v_{i r} \frac{\partial \eta_{e 0}}{\partial r}
$$

Implementing this change simply "turns off" the horizontal components of the partial derivatives of the ionospheric wave and background electron density. Plugging 
Equation (2.8) back into Equation ( 2.5) we obtain:

$$
\partial n_{e}=-\int_{0}^{t} n_{e 0}\left[\frac{2}{r} v_{i r}+\frac{\partial v_{i r}}{\partial r}\right]+v_{i r} \frac{\partial \eta_{e 0}}{\partial r} d t
$$

This represents a model where the $n_{e 0}$ term varies only in the radial direction, as does the plane wave in the analytical model.

\subsubsection{Line of Sight Integration}

The methods described in the previous section give the change in the electron density at any point in the ionosphere. In order to compare the 1D and 3D models with observed TEC we need to integrate along the line of site (LOS), L, of the receiver and GPS satellite so that:

$$
T E C=\int_{L O S} \partial n_{e} d r
$$

I show an example LOS and resulting TEC from integration in Figure 2.1b and 2.1c.

I combine Equations ( 2.5) and ( 2.7) or (2.8) for the 3D or 1D cases, respectively. The $1 \mathrm{D}$ case is:

$$
T E C=\int_{L} \partial n_{e 0}(\mathbf{r}, \mathbf{t}) d r=\int_{L}-\left[\int_{0}^{t} n_{e 0}\left[\frac{2}{r} v_{i r}+\frac{\partial v_{i r}}{d r}\right]+v_{i r} \frac{\partial \eta_{e 0}}{\partial r} d t\right] d r
$$

In summary, for this method, I numerically integrate over time to find the change in the electron density as a function of position. Then I integrate over the LOS to obtain TEC. In the following section I describe the analytical model developed by Georges \& Hooke (1970), which replaces the time and LOS integration with a 1D analytical approximation. I go through the same 3 steps as in the current section, beginning with the acoustic wave. 


\subsection{Analytical Model}

\subsubsection{Acoustic Wave: Plane Wave Approximation}

If our observation point is far enough away from the source we can assume a plane wave. Georges (1968) develops such an approach using a plane wave approximation of the neutral atmospheric wave:

$$
\mathbf{v}_{\mathbf{n}}(\mathbf{r}, t, \omega) \approx V(z) e^{i(\mathbf{k} \cdot \mathbf{r}-\omega t)}
$$

where $V(z)$ is the height dependent amplitude, $\omega$ is angular wave frequency, and $\mathbf{k}$ is the wave vector with phase velocity $c$. The electron density perturbation in the ionosphere is similarly planar. During the atmosphere-ionosphere coupling, the phase of the acoustic wave is conserved; only the amplitude changes according to the coupling factor.

\subsubsection{Ionospheric Coupling}

Georges (1968) also assumes that the horizontal gradient of the background electron density is negligible and using the continuity equation (Equation 2.3) obtains a planar continuity equation:

$$
\partial n_{e}(\mathbf{r}, t)=\frac{1}{\omega}\left[\mathbf{v}_{\mathbf{n}}(\mathbf{r}, t) \cdot \hat{\mathbf{b}}\right]\left[(\mathbf{k} \cdot \hat{\mathbf{b}}) n_{e 0}(z)+i(\hat{\mathbf{b}} \cdot \hat{\mathbf{z}}) \frac{\partial}{\partial z} n_{e 0}(z)\right]
$$

Note that this planar continuity equation only includes a 1-D gradient of the background electron density (in the vertical direction). This method also removes the integration over time to compute $\partial n_{e}$. 


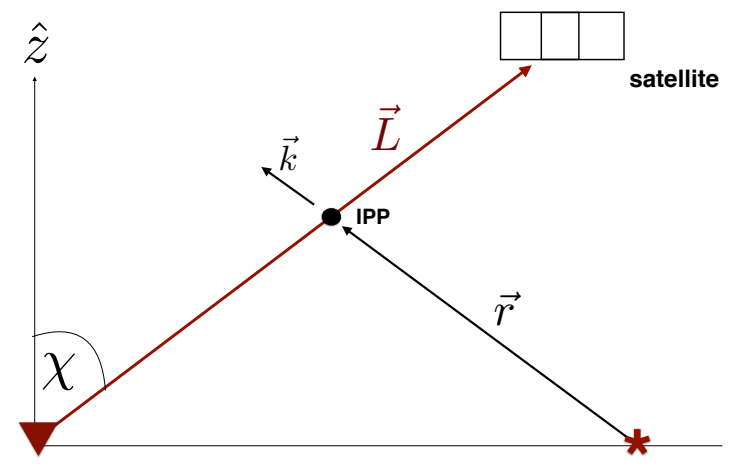

Figure 2.2: Diagram of the relationship between $\mathrm{L}, \mathrm{K}, \mathrm{r}$, and $\chi$. The red triangle is the ground receiver and $*$ is the source location.

\subsubsection{Line of Sight Integration}

Georges \& Hooke (1970) derive the TEC by integrating Equation (2.12) along the LOS of the satellite-receiver pair. They obtain the following equation:

$$
T E C=\left[u e^{i(\omega t+\mathbf{k} \cdot \mathbf{r})}\right]\left[\frac{1}{\omega \cos ^{2}(\chi)}\right][(\hat{\mathbf{k}} \cdot \hat{\mathbf{b}})(\hat{\mathbf{r}} \times \hat{\mathbf{b}}) \times \hat{\mathbf{z}} \cdot \mathbf{k}]\left[\int_{-\infty}^{\infty} n_{e 0}\left(h_{m}+z^{\prime}\right) e^{\left(i \eta z^{\prime}\right)} d z^{\prime}\right],
$$

where: $\chi=\cos ^{(-1)}(z / c)$ is the zenith angle of the LOS $(\mathbf{L})$ and is shown in Figure (2.2), $z^{\prime}=\left(z-h_{m}\right), h_{m}$ is the altitude of the peak electron density, and $\eta=\frac{\mathbf{k} \cdot \mathbf{r}}{h_{m}}$.

This equation still includes an integration term. The authors give several analytical approximations of the ionospheric profile, including an $\alpha$-chapman approximation, where the normalized background electron density is given by:

$$
\frac{n_{e 0}}{n_{e m}}=e^{\frac{i}{2}\left(i-\frac{z^{\prime}}{H}-e^{-z^{\prime} / H}\right)}
$$

where $n_{e m}$ is the maximum electron density. Using equation 2.14 and solving the integral term in Equation 2.13 they obtain: 


$$
\left.\int_{-\infty}^{\infty} n_{e 0}\left(h_{m}+z^{\prime}\right) e^{\left(i \eta z^{\prime}\right)} d z^{\prime}\right]=\frac{2^{-i \eta H}}{\sqrt{\pi}} \Gamma\left(\frac{1}{2}-i \eta H\right)
$$

where $\Gamma$ is the gamma function of complex argument and $\mathrm{H}$ is the thickness of ionosphere. Equation 2.13 then becomes

$$
T E C=\left[u e^{i(\omega t+\mathbf{k} \cdot \mathbf{r})}\right]\left[\frac{1}{\omega \cos (\chi)^{2}}\right][(\hat{\mathbf{k}} \cdot \hat{\mathbf{b}})(\hat{\mathbf{r}} \times \hat{\mathbf{b}}) \times \hat{\mathbf{z}} \cdot \mathbf{k}]\left[\frac{2^{-i \eta H}}{\sqrt{\pi}} \Gamma\left(\frac{1}{2}-i \eta H\right)\right]
$$

This is the final equation for the TEC using the Georges and Hooke model.

Here I review the four terms given above in between brackets. Term one is the result of the interaction of the acoustic wave with the ionosphere. Term two accounts for the satellite elevation. Term three includes the influence of the geometry of the IPP with respect to the source, direction of propagation, and Earth's magnetic field. Figure 2.2 shows the relationship between the IPP, $\mathbf{k}, \mathbf{r}$ and the line of sight (LOS). Finally, the fourth term is the phase cancellation term. Depending on the geometry of the line of sight and the ionospheric wave, the integration term can sum the TEC constructively or destructively and cause changes to the polarity and phase of the TEC signal. 


\section{CHAPTER 3:}

\section{TEC SENSITIVITY TO MODEL PARAMETERS}

\subsection{Chapter Summary}

In Chapter 2, I outlined the methods used to model the TEC developed in Rolland et al. (2011). To move toward an analytical model such as that employed by Gõmez et al. (2015), I test the assumption that the background electron density varies in one dimension. I run a modified version of the spherical wave model that allows me to use a $3 \mathrm{D}$ or $1 \mathrm{D}$ vertical divergence in the step to calculate the electron density perturbations. For each case, I run the perturbation step and then integrate along a LOS to get a corresponding 1D or 3D TEC time series. I then compare the differences in the waveforms by finding the root mean square (RMS) difference between the self-normalized time series for synthetic station. I present the results for a Northern Hemisphere mid-latitude location in section 3.3.1. I further test the effect of the geomagnetic field on the electron density by running the divergence analysis at different latitudes. I use test locations at mid and high latitudes for both hemispheres as well the Equator during the month of October. 


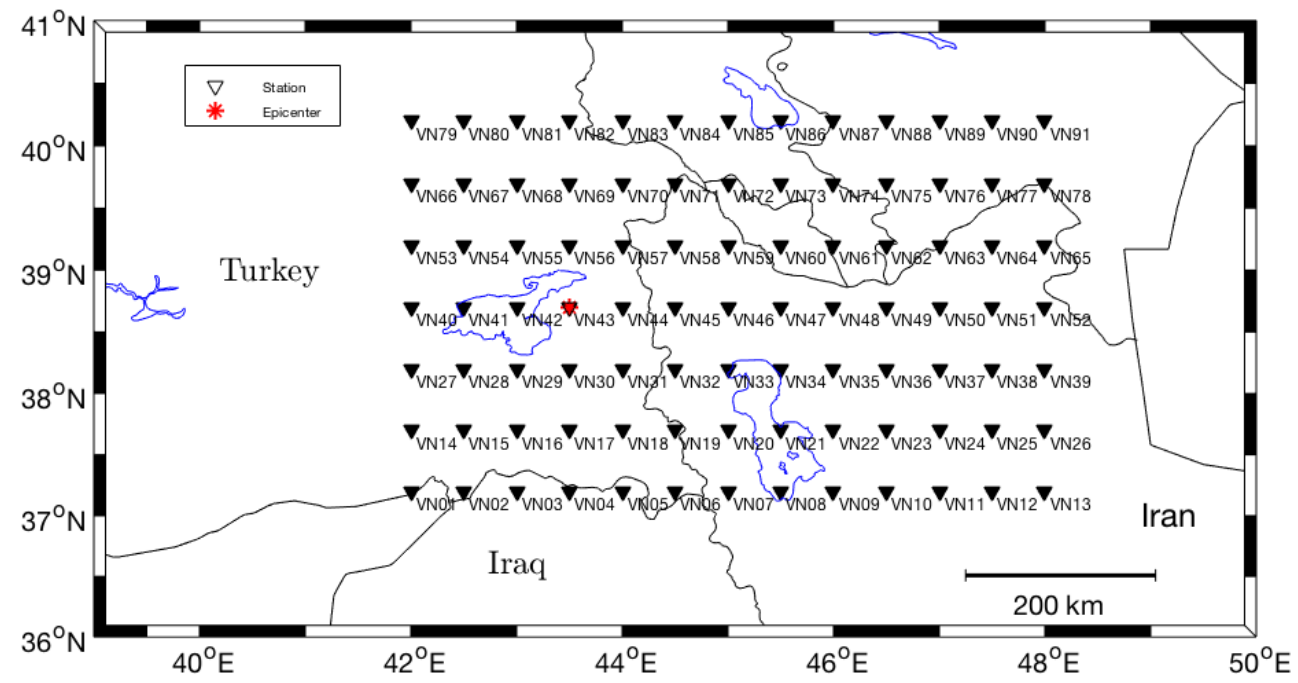

Figure 3.1: Station grid for the Van location. Other locations have grids with similar geometry.

\subsection{Methods}

\subsubsection{Station Grid}

For each test location, I use a grid of stations that is $333 \mathrm{~km}$ north to south and $522 \mathrm{~km}$ east to west. Stations are separated by $\sim 43.5 \mathrm{~km}$ in the longitude direction and $55.5 \mathrm{~km}$ in the latitude direction. The epicenter is approximately in the middle latitude and one quarter the total distance in the longitude direction. This produces a grid that is $7 \times 13$ stations. Figure 3.1 shows the station layout for an epicenter location at the site of the $2011 \mathrm{M}_{\mathrm{w}} 7.1$ Van earthquake $\left(38.7^{\circ} \mathrm{N}, 43.5^{\circ} \mathrm{E}\right)$. This was the site of the study in Rolland et al. (2013) in which the model successfully reproduced the observed TEC (see Figure 1.6a). Here, I use synthetic stations that match the spatial extent of actual stations in the area and is therefore a realistic, if somewhat 
Table 3.1: Earth's magnetic field at the 4 test locations. Longitude for all locations is $43.5^{\circ} \mathrm{E}$.

\begin{tabular}{|c|c|c|c|c|}
\hline Location & Latitude $\left(^{\circ}\right)$ & Inclination $\left(^{\circ}\right)$ & Declination $\left(^{\circ}\right)$ & IPP height $(\mathrm{km})$ \\
\hline N High Lat & 75 & 82 & 20 & 300 \\
\hline N Mid Lat & 38.7 & 57 & 5 & 260 \\
\hline Equator & 0 & -18 & -1 & 380 \\
\hline S Mid Lat & -38.7 & -62 & -37 & 290 \\
\hline S High & -75 & -68 & -57 & 260 \\
\hline
\end{tabular}

idealized, station grid. Text beside each station gives station names.

\subsubsection{Grid Locations}

Earth's magnetic field has an important impact on the TEC (Heki et al., 2006). Perturbations from the acoustic wave couple to the ionosphere and transfer momentum into electrons parallel to the Earth's magnetic field. I test this effect on the 1D divergence assumption by using the same grid at mid and high latitudes, and the Equator. I use the same longitude for all locations. For the mid latitude epicenters I use the 2011 Van earthquake location for the Northern Hemisphere (hereafter N Mid Lat) and -38.7 degrees for the southern latitude (S Mid Lat). For the high northern (N High Lat) and southern (S High Lat) latitudes I use \pm 75 degrees, respectively. Table 3.1 shows the inclination and declination at each of these locations along with the IPP height used. For the IPP height, I use the maximum electron density height for each location.

\subsubsection{Satellite Positions}

I use actual GPS satellite positions to calculate the LOS and integrate the TEC. I choose the satellites to use by comparing elevation angles for all satellites. For each grid location, I examine the elevation angle of an imaginary station at the epicenter 

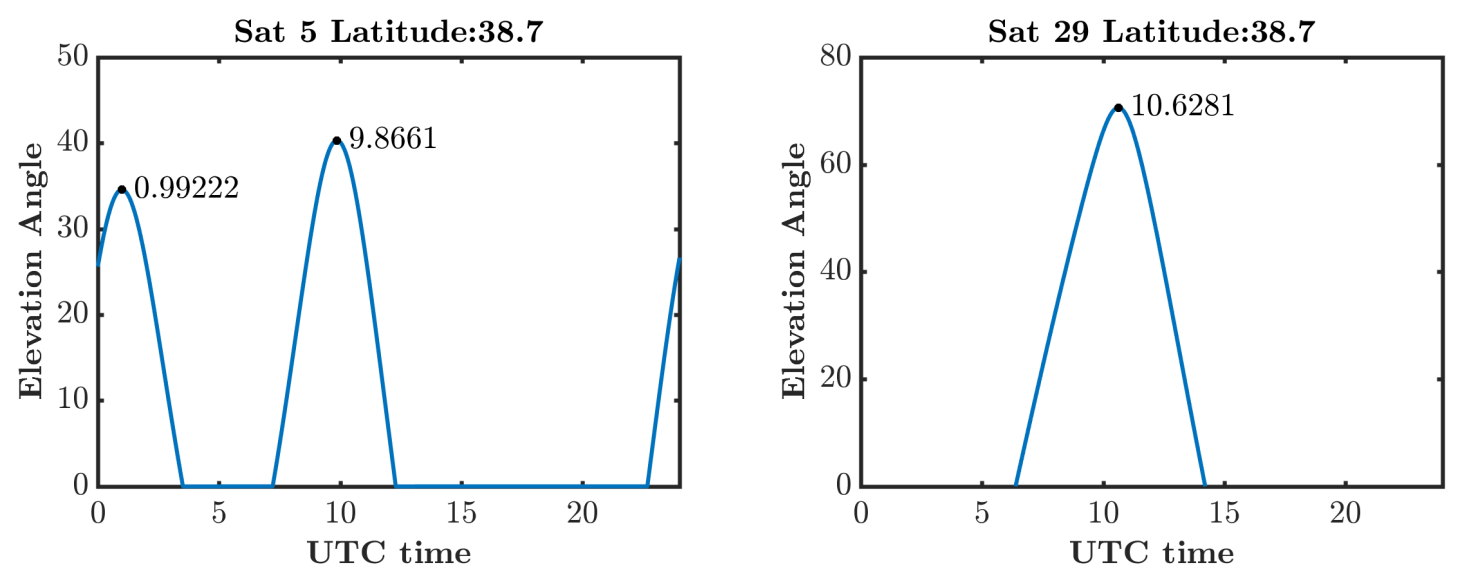

Figure 3.2: Examples of the elevation angles for 2 satellites at the $\mathrm{N}$ Mid Lat location. Elevation of angles of zero are when the satellite was below the horizon.

with all satellites during the day of October 23, 2011. Examples of the elevation angles for 2 satellites at the N Mid Lat location are shown in Figure 3.2. For all but the N High Lat, I chose a time during daylight hours that is close to the time of the maximum elevation angle for 2-3 satellites. The N High Lat was in 24 hour darkness during this time. The times and satellites chosen are presented in Table 3.2.

Table 3.2: Times and satellites used to model the TEC at each test location.

\begin{tabular}{|c|c|c|}
\hline Location & Time (UTC) & Satellites \\
\hline N High Lat & $10: 50$ & G5, G29 G30 \\
\hline N Mid Lat & $10: 50$ & G5, G21, G25, G29 \\
\hline Equator & $10: 50$ & G18, G21, G25 \\
\hline S Hemisphere & $9: 17$ & G09, G18, G27 \\
\hline S High Lat & $9: 17$ & G09, G18, G27 \\
\hline
\end{tabular}




\subsection{Results}

\subsubsection{Impact of Electron Density Divergence on TEC}

I show an example of the modeled TEC for 1D and 3D divergence for two stations in Figure 3.3. Both waveforms are for the N Mid Lat grid location for satellite 21 (G21, where G indicates a GPS constellation satellite for all satellites hereafter). Station 8 (top) is an example where the 1D divergence has significant phase differences, whereas station 25 (bottom) shows some amplitude difference but fits the phase. In Appendix A, I show analysis of the misfit that shows that energy is conserved between the 1D and $3 \mathrm{D}$ divergence, but that the phase varies significantly. See Figure 3.1 for station locations.
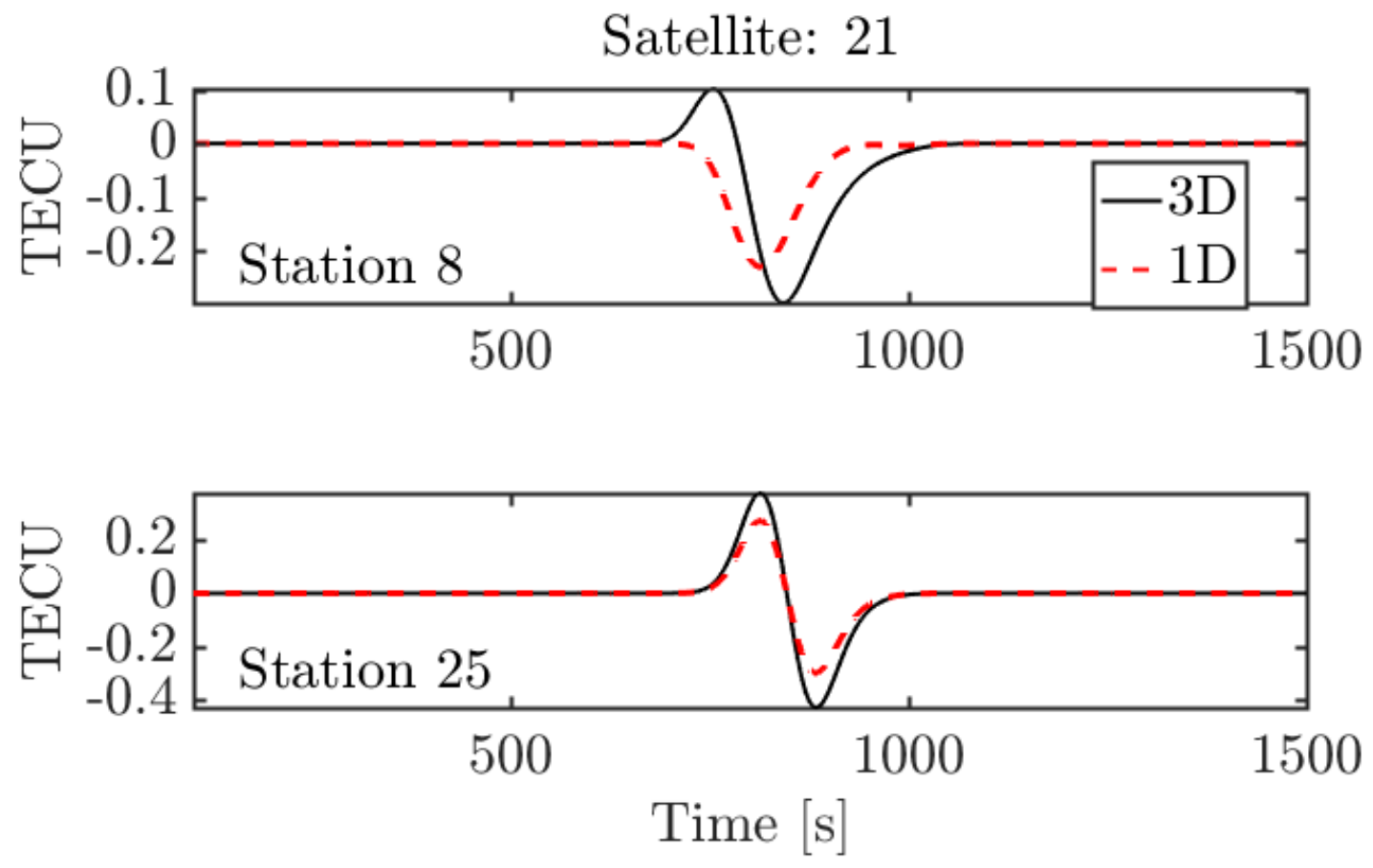

Figure 3.3: Waveform comparisons of $1 \mathrm{D}$ and $3 \mathrm{D}$ divergence results of modeled TEC. Stations are from the N Mid Lat grid and paired with the same satellite (G21). 
Figure 3.4 shows the elevation angles for all stations (right column) as well as the RMS (left column) for 4 of the 5 satellites in view at 10:50 am. Satellite numbers are given for each plot and are in order of decreasing elevation angles from top to bottom. For each satellite except G05, I found the elevation angle for all stations at time $\mathrm{t}=600$ seconds. This time corresponds with the arrival times in the modeled TEC time series. Satellite G05 had overall lower satellite angles during the minutes after the modeled event. The lower angles produce IPPs further from the stations and thus later arrival times. Therefore, I use a time consistent with arrival times for G05 of 1000 seconds to plot the elevation angle. Together, the four satellites cover a range of elevations from about 34 degrees up to a high of about 76 degrees (G29). Areas with the highest elevation angle in each plot indicate the direction that IPPs are shifted relative to the stations on the ground. Satellites G05 and G29 have IPPs to the northeast of the stations while G21 and G25 are shifted to the west and south, respectively.

The RMS differences for all satellites was low (see left column, Figure 3.4). Only satellite G21 showed an error above 15\%. See station 8 in Figure 3.3 and 3.4 for an example of a high-error station contrasted with a station (25) with smaller waveform differences. The highest error was $32 \%$ and is in the north east corner. The fifth satellite in view, G30 (see appendix, Figure C.1) had similar elevation angles and RMS to G21.

\subsubsection{Magnetic Field Influence on TEC}

I explore the effect of the Earth's magnetic field by conducting the same analysis on the divergence of the electron density for 4 other latitudes. I show the results for these locations in Figures 3.5 - 3.8. With the exception of G10 (which had very low 

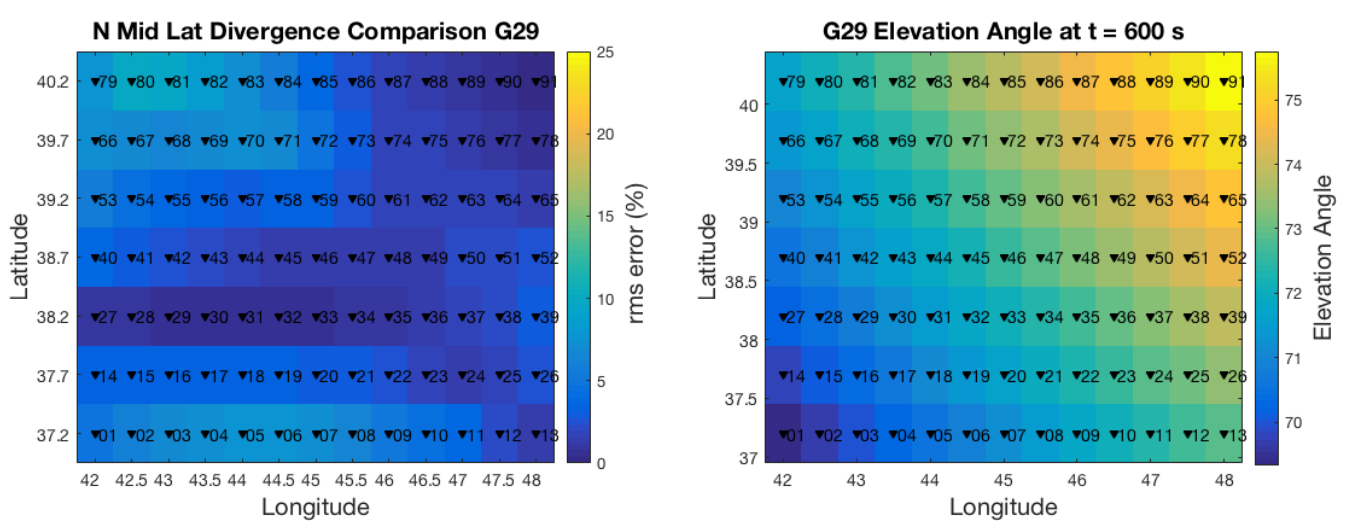

33
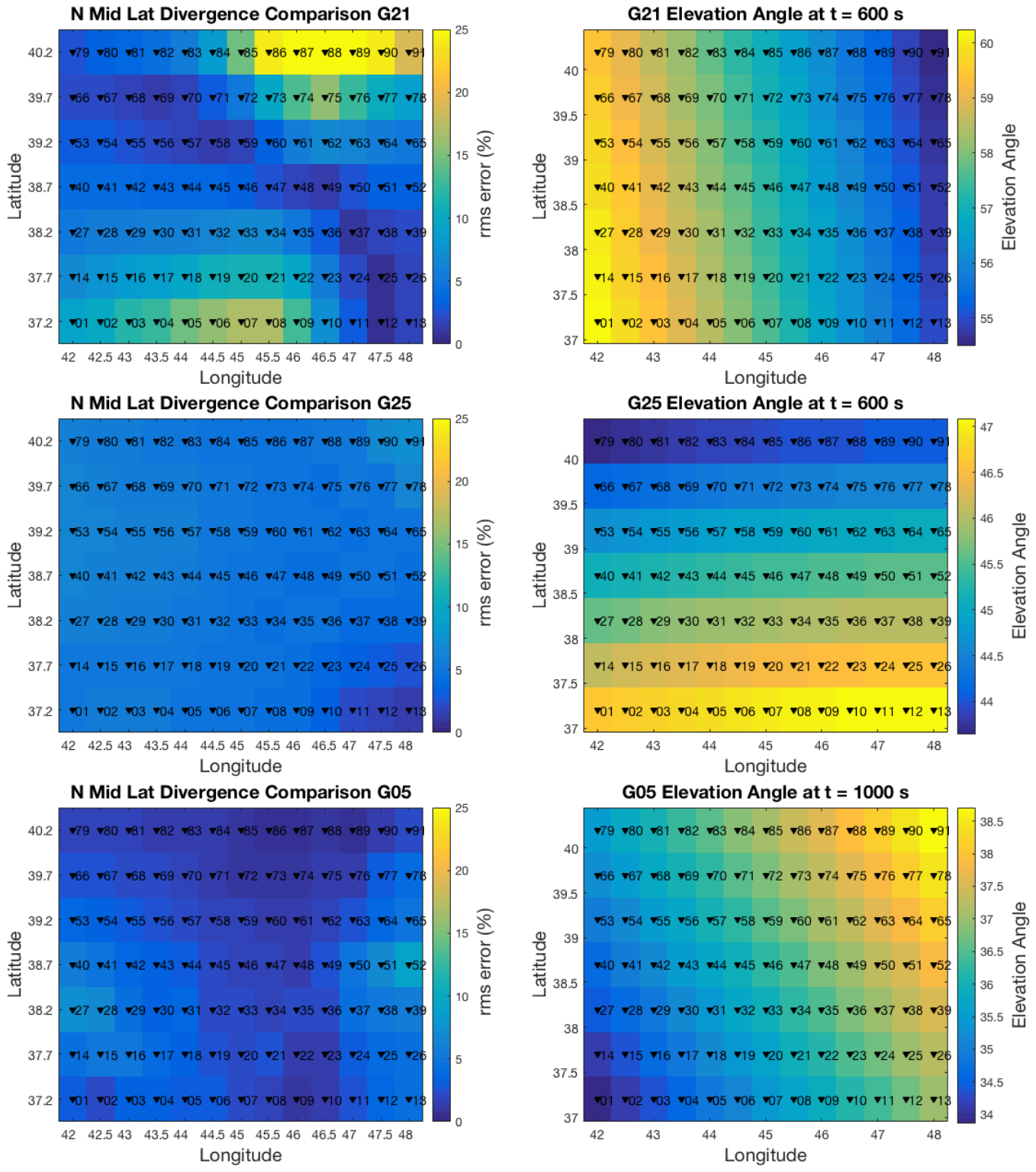

Figure 3.4: Waveform differences and elevation angles for N Mid Lat grid location. 
elevation angles), the N High Lat (see Figure 3.5) was the only other location with consistently low RMS differences, indicating that the vertical divergence is adequate for modeling purposes.

At the Equator (Figure 3.6), the two higher elevation satellites (G25 and G18) have some areas with lower error, while the low angle satellite (G21) shows the highest phase difference with over $60 \%$ difference in the RMS. Overall, the equator gave the highest RMS error of all locations.

At the S Mid Lat location (Figure 3.7), two satellites (G09 and G27) have IPPs to the southeast of the stations but with different elevation angles. Satellite G09 shows the lowest RMS error of the two and has higher elevation angles (50-55 vs. 35-40).

Results for the S High Lat (Figure 3.8) are also mixed. Satellites G09 and G27 have relatively low RMS error with areas of higher error. Both show a similar pattern in high error areas in the west part of the grid. The slight difference in the direction of the LOS (G27 to the east and G09 to the northeast) may account for the increased wave differences on the eastern side of the grid for G27.

I show global electron density at the peak electron density height of $280 \mathrm{~km}$ for October 23 at 10:50 UTC in Figure 3.9. The distribution of the electron density is approximately symmetrical for the locations tested (locations indicated by $*$ ). Initial investigation showed no correspondence to the amplitude or gradient of the electron density along satellite-receiver LOS with RMS differences. 

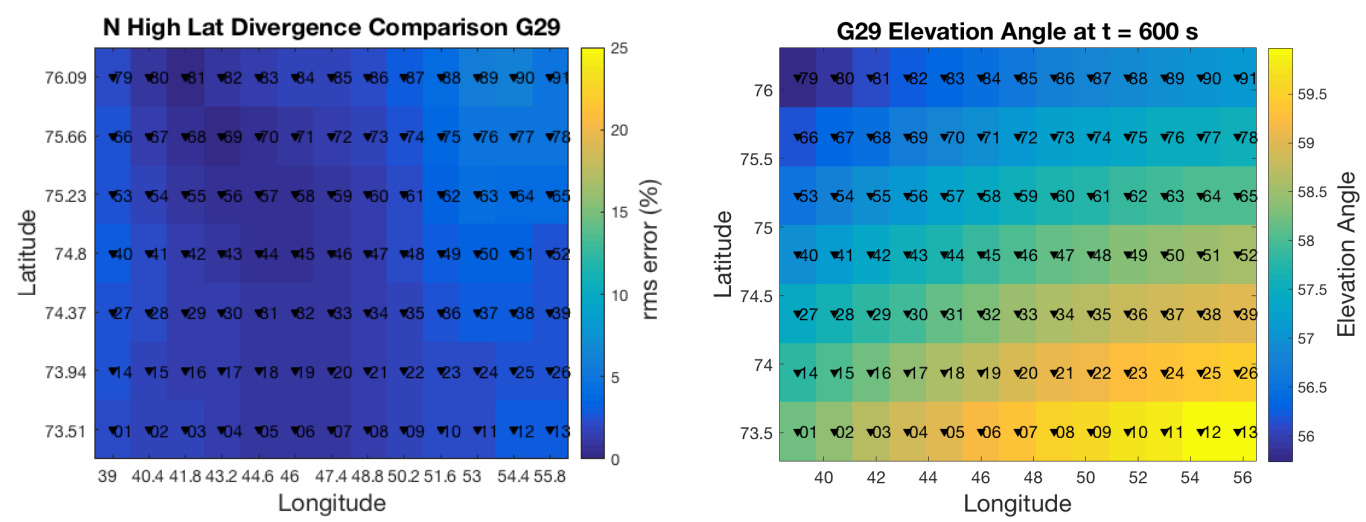

35
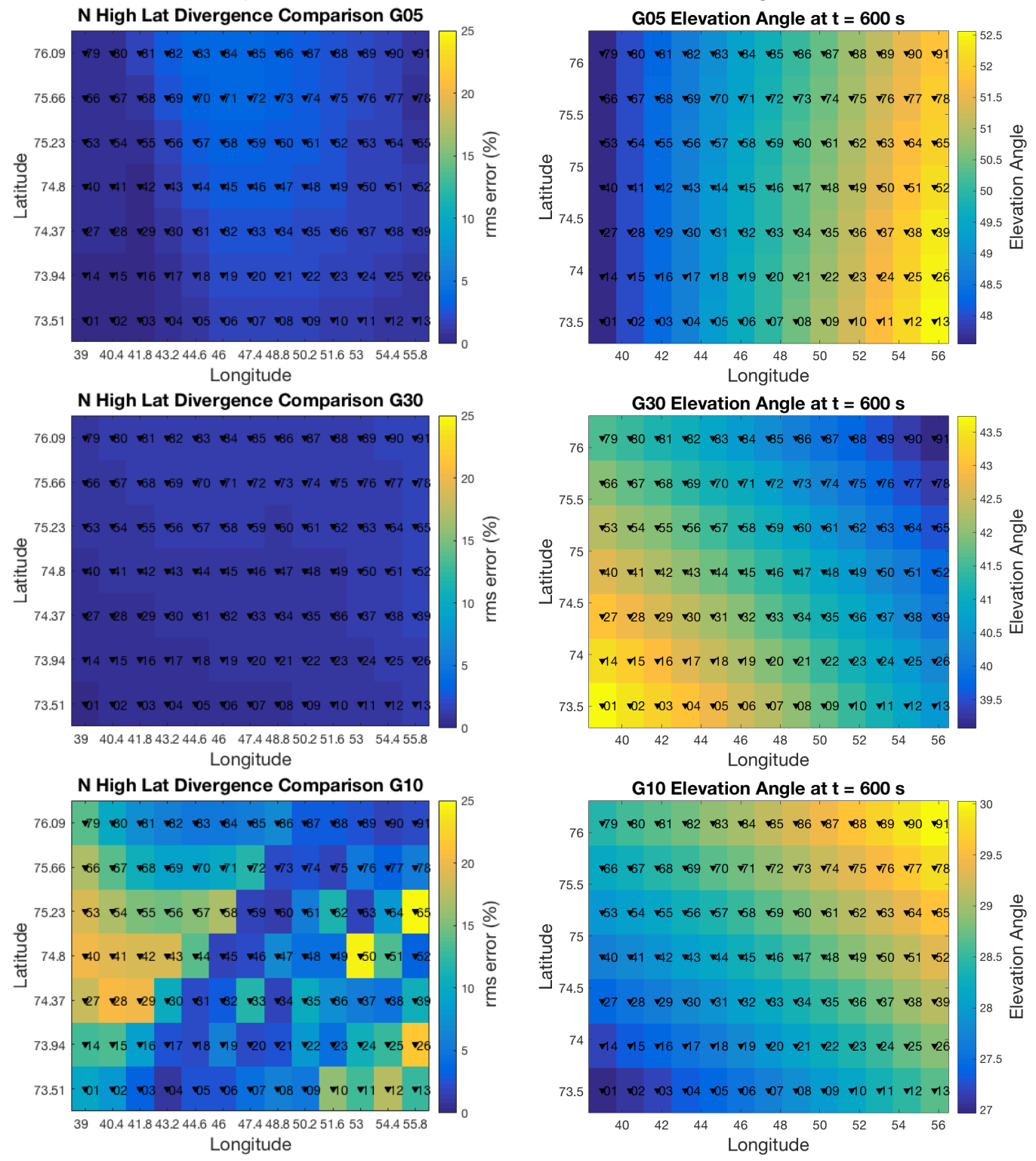

Figure 3.5: Waveform differences and elevation angles for $\mathrm{N}$ High Lat. 


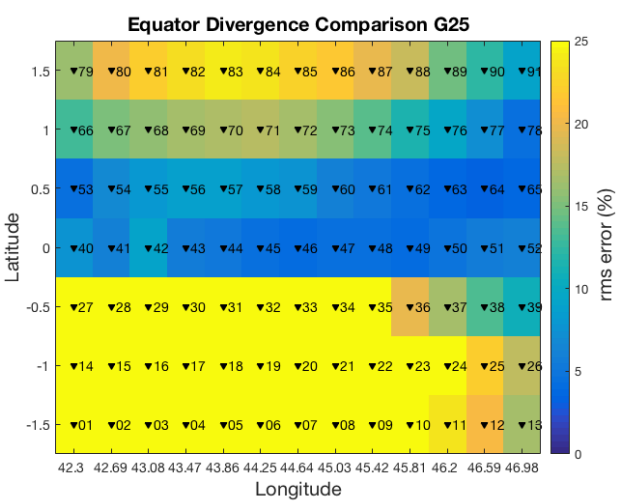

Equator Divergence Comparison G18

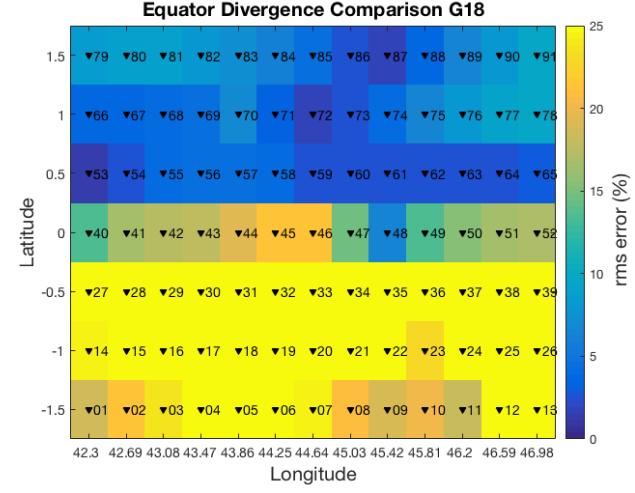

Equator Divergence Comparison G21

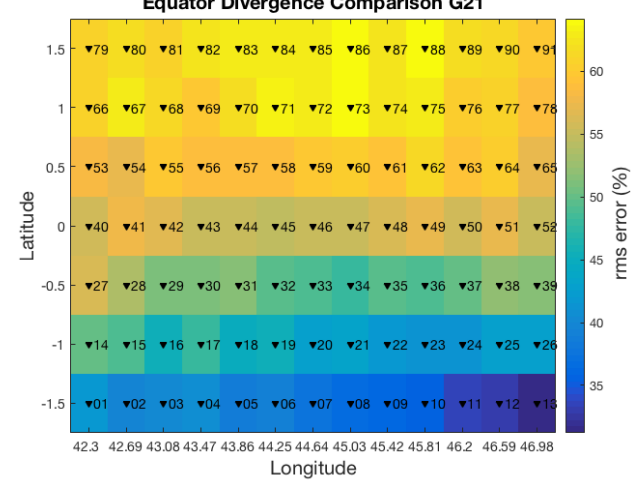

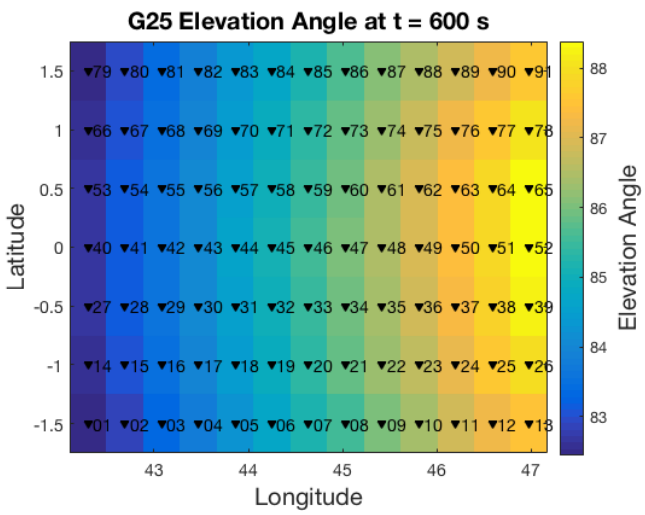

G18 Elevation Angle at $\mathrm{t}=600 \mathrm{~s}$
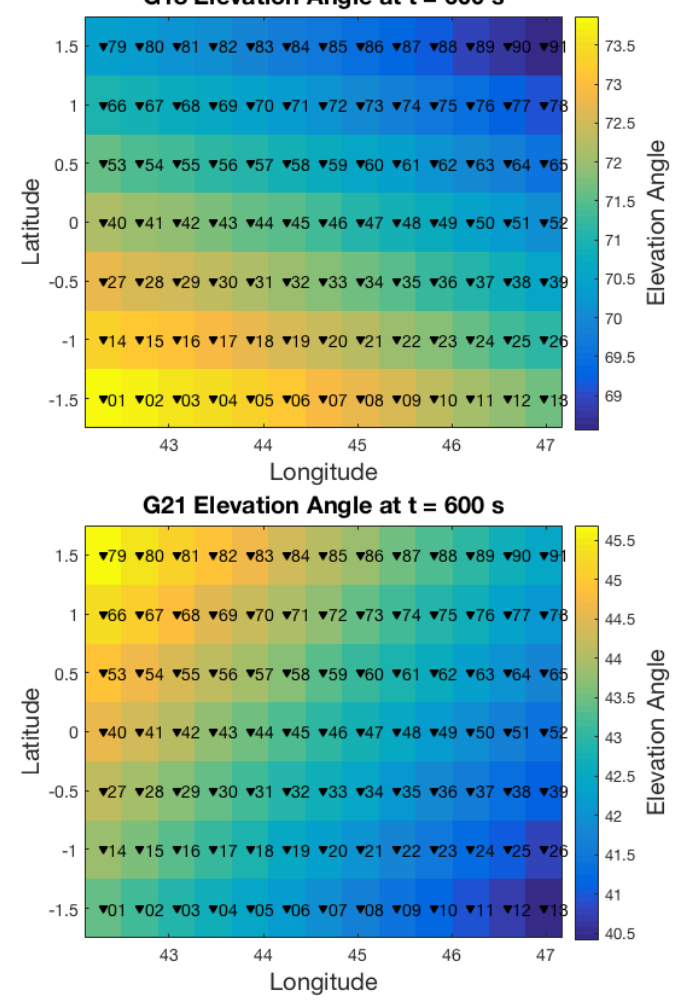

Figure 3.6: Waveform differences and elevation angles for Equator. Note change in the color axis in G21 to show higher errors. 

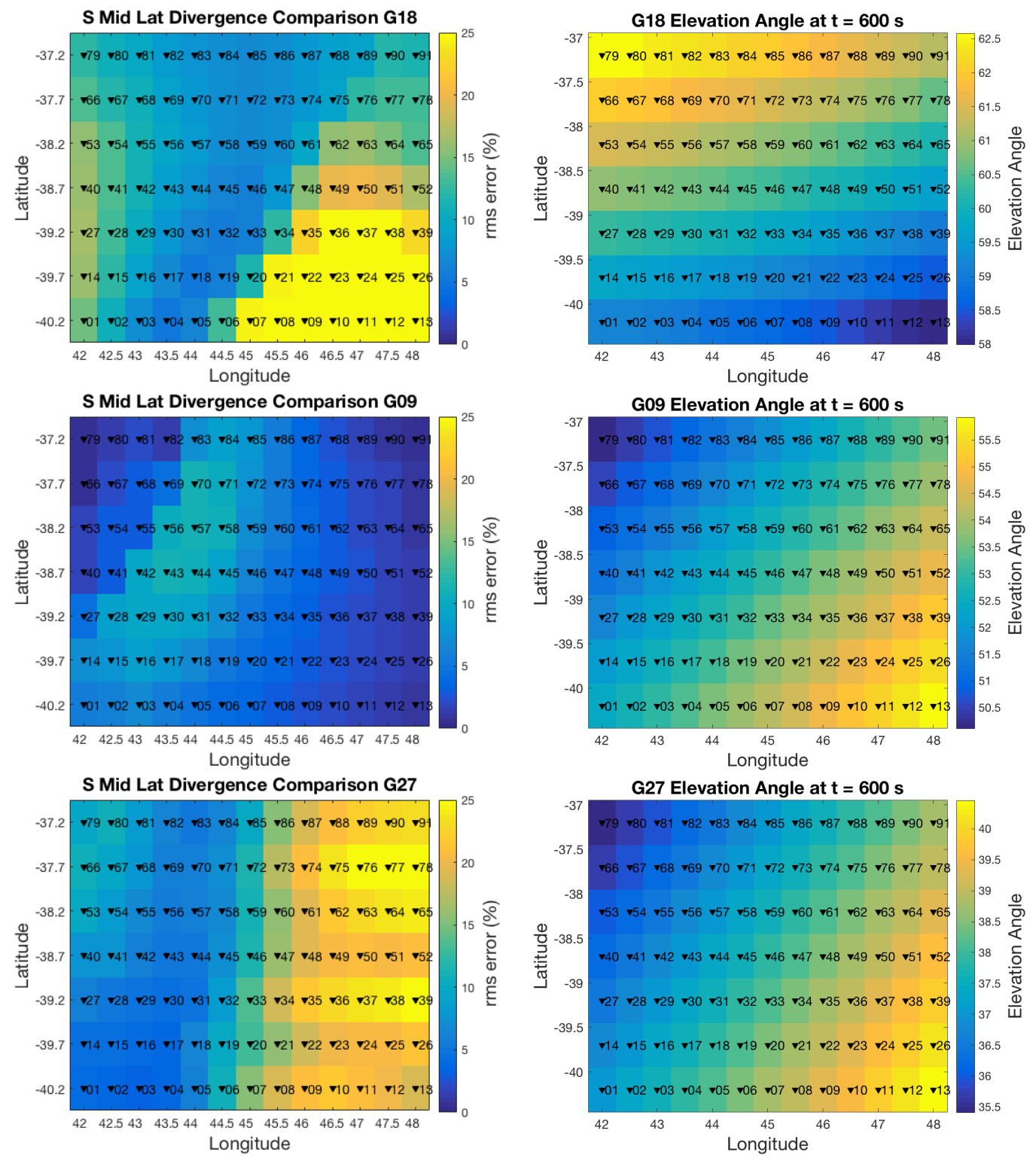

Figure 3.7: Waveform differences and elevation angles for S Mid Lat. 


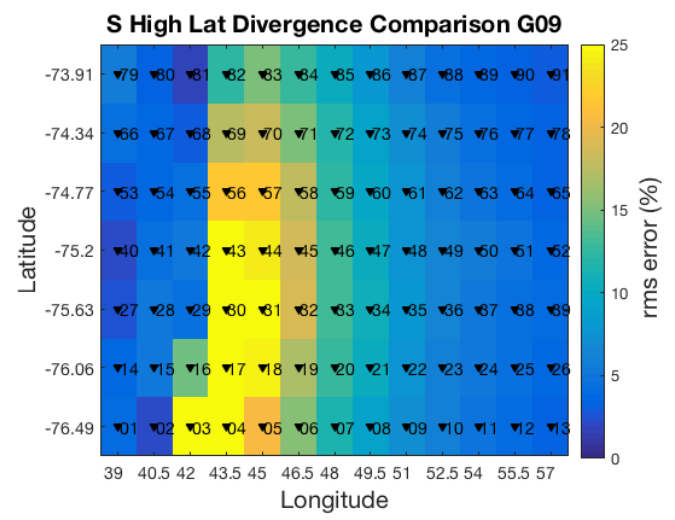

S High Lat Divergence Comparison G27
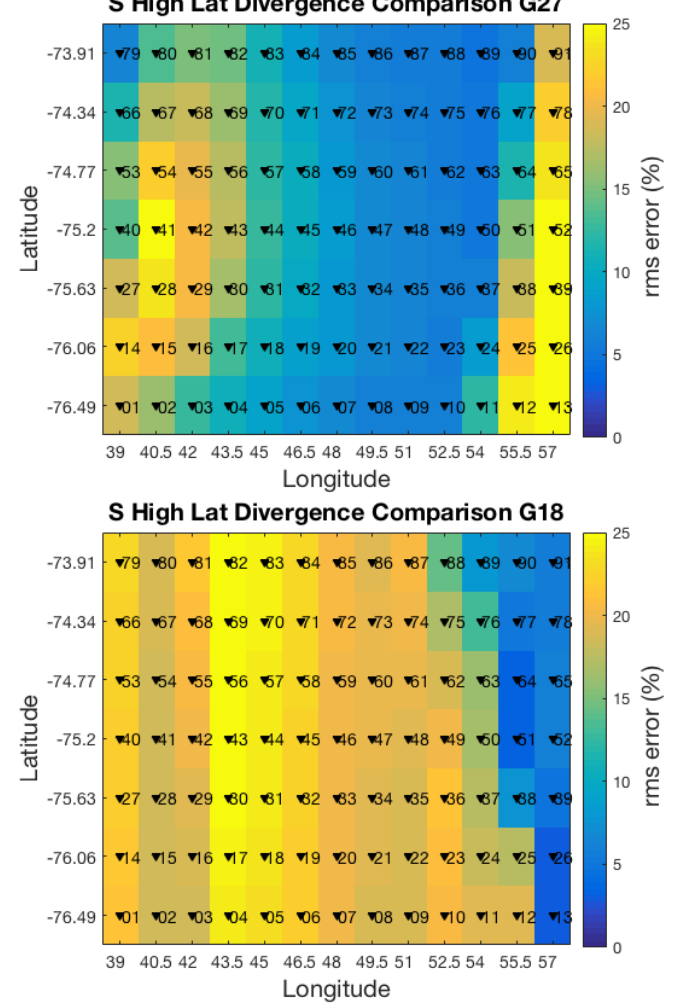

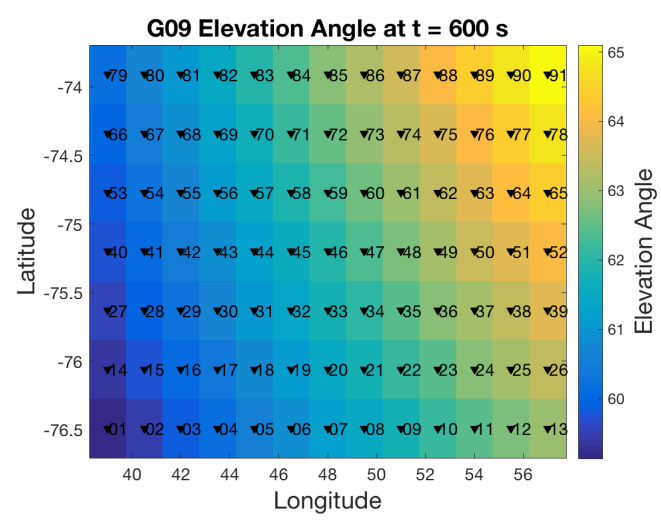

G27 Elevation Angle at $\mathrm{t}=\mathbf{6 0 0 \mathrm { s }}$

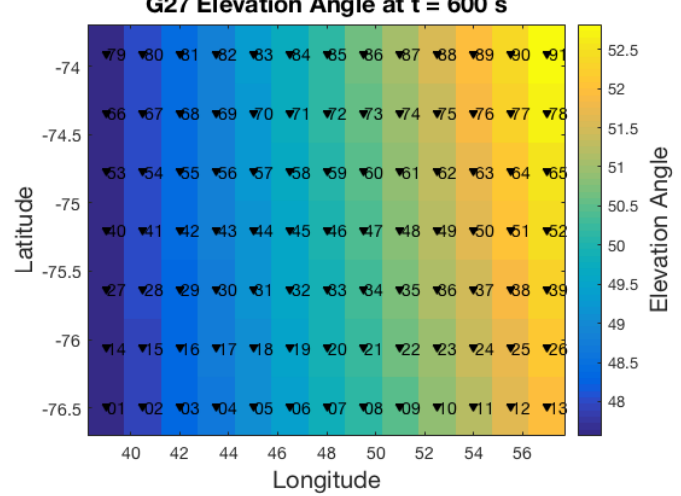

G18 Elevation Angle at $\mathrm{t}=600 \mathrm{~s}$

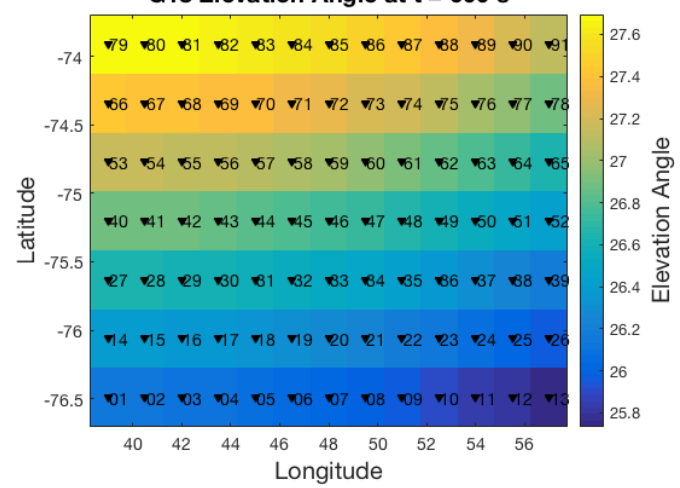

Figure 3.8: Waveform differences and elevation angles for S High Lat. 


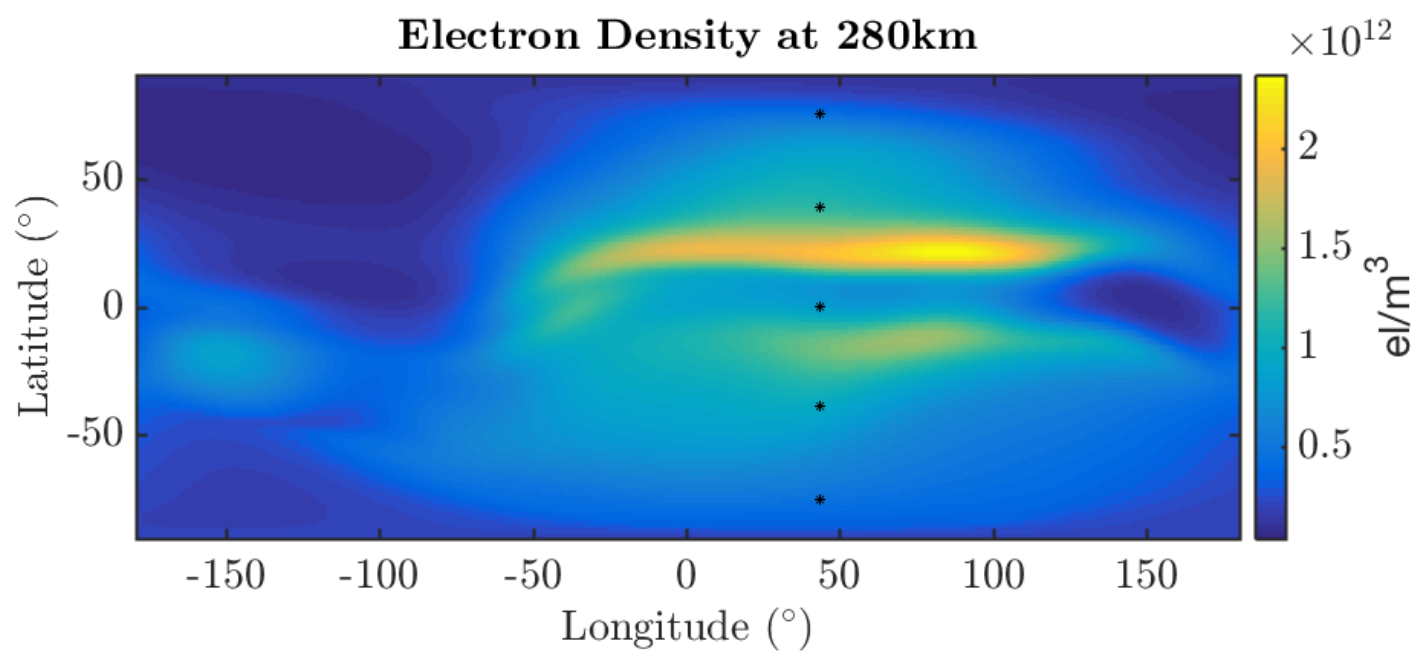

Figure 3.9: Global background electron density on October 23, 2011 at 10:50am.

\subsection{Discussion}

The results from the modeling of the $\mathrm{N}$ Mid Lat location indicate that, in this case, a 1D approximation of the divergence is a reasonable assumption. Elevation angle had no apparent effect on the RMS differences. Results for the highest elevation angle (G29) were similar to those with the lowest (G05). The general direction from both these satellites was similar (to the northeast). Satellite G21 had the highest error and was the only satellite with LOS to the west of the stations. This suggests that the direction of the LOS is more important than elevation angle.

Results for the other test locations indicate low RMS error for both Northern Hemisphere locations (N High Lat and N Mid Lat). The higher error in G10 for the N High Lat indicates that very low elevation angles (less than $30^{\circ}$ in this case) introduce error not present in any elevation above the cut-off elevation angle.

At the Equator and in the Southern Hemisphere results are more mixed. Some satellites show good correspondence between the 1D and 3D divergence as with G09 
in the S Mid Lat location (Figure 3.7). A second satellite (G27), with similar LOS, but lower elevation angle has higher error. One interpretation of this result is that elevation angles have more influence in the Southern Hemisphere than in the Northern Hemisphere. However, the influence of the elevation angle is still secondary to the LOS as high elevation angles at the equator (G18 and G25 in Figure 3.6) do not yield lower error. The importance of a low cut off angle holds in both Northern and Southern Hemispheres. As with the N High Lat, elevation angles under $30^{\circ}$ in the $\mathrm{S}$ High Lat produce more overall error (G18, Figure 3.8). For the Equator (the location with highest overall error) the cut-off for increased relative error is higher, with G21 showing markedly increased error under $45.5^{\circ}$

Here I only observe the differences between the Northern and Southern Hemispheres. However, one explanation for the differences may be in the seasons. The tilt of the earth relative to the sun creates a difference in how direct the sun's rays are in the Northern and Southern Hemispheres. During summer in the Northern Hemisphere, the Northern Hemisphere is tilted toward the sun whereas the Southern Hemisphere is titled away, because radiation from the sun is what strips electrons away from neutral atoms (thus creating the ionosphere), this difference in the seasons may be what is causing the 3D difference in the Southern Hemisphere in October.

Examining the structure of the ionospheric electron density at the height of maximum electron density did not show any significant differences between the two hemispheres for the day tested. However, to better understand the influence of the seasons on the 3D divergence the analysis conducted here should be repeated for different times of year. 


\subsection{Conclusion}

For this thesis, I investigate the cause of the patterns in the RMS differences by examining the elevation angles and receiver-satellite LOS as well as the global background electron density. While the LOS does impact the results, the relationship with the ionospheric structure is unclear. As discussed in Chapter 2, the coupling factor (Equation 2.3) is a fundamental description of atmospheric-ionospheric coupling. Therefore, a third plot of the projection of the acoustic wave onto the magnetic field could yield further insights.

These initial observations show that the reliability of the 1D assumption is com-

plex. For an event in fall, the 1D assumption holds in the Northern Hemisphere. However, the direction of the LOS also plays a role and can introduce higher errors in small areas. Elevation angle has little impact on the results except at very low angles. This elevation angle cut off is higher at low latitudes near the Equator.

I suggest several lines of research for additional study. First, a more detailed investigation of the relationship of the $1 \mathrm{D}$ vs $3 \mathrm{D}$ waveform differences with the LOS. For this study, I used actual satellite locations. Synthetic simulation of satellite positions would enable testing of the IPPs with the same or similar elevation angles but with different directions of the LOS. Additionally, plots of the coupling factor at each IPP may better explain the relationship of the LOS with ionospheric structure.

Second, an investigation of the cut-off angle would be useful. This would need to be tested at more latitudes to gain an understanding of how the cut-off angle might vary with latitude. Finally, a temporal study would investigate the relationship between the seasons and the errors in the two hemispheres. For example, would the errors be higher in the Northern Hemisphere during the spring and summer? 


\section{CHAPTER 4:}

\section{TEC EXTRACTION}

In the previous chapter, I outlined the process to forward model the TEC. Here I describe the process to collect observed data from GNSS. First I describe how to download GNSS data. I include a brief overview of GNSS constellations, file formats and how to find GNSS data online. Then I explain how to extract the TEC using a software maintained by UNAVCO. I also give a brief overview of the workflow we use to extract TEC from GPS data. Next, I describe how to obtain coordinates for the height dependent IPP. Finally, I give an example of the processing we use in the next Chapter once we have extracted the TEC.

\subsection{Download GNSS data}

There are multiple GNSS constellations in orbit. The global positioning system (GPS) is maintained by the US Air Force. Other GNSS constellations include GLONASS (Russia), Galileo (European Union) and BeiDou (China). Data from these constellations (especially GPS and GLONASS) are easily available on the internet. Below I list just a few of those sites. Another method to find data is to search for the region of interest. The standard file format for GNSS data is the Receiver Independent Exchange (RINEX). As an example, a search for "GPS rinex Italy" will return a result for the RING network (link below) along with other websites offering GNSS datafiles. 
The files online are compressed in the Hatanaka format. Observation files contain the GNSS data and navigation files contain satellite positions. We require both to extract the TEC.

\section{Example GNSS Websites:}

- UNAVCO has a user friendly interface with an optional demo for new users at:

http://www .unavco.org/data/gps-gnss/data-access-methods/dai2/app/dai2.html\#

- SOPAC

http://sopac.ucsd.edu/sopacDescription.shtml

\section{- RING network}

http://ring.gm.ingv.it/

\subsection{TEC Extraction}

UNAVCO maintains a toolkit for preprocessing GNSS called teqc (pronounced "tek"). The three main functions from which the program derives its name are translation, editing and quality check. Teqc can process multiple satellite constellations including GPS, GLONASS and Galileo and can handle many native receiver formats. Teqc is available for most operating systems and can be downloaded at the following url: https://www . unavco.org/software/data-processing/teqc/teqc.html.

Before processing the data, Hatanaka files must be converted into RINEX. If the data is compressed, teqc can perform the decompression from Hatanaka to RINEX. Alternatively, RNXCMP software will compress or restore RINEX observation files. This software was developed by Y. Hatanaka and is available at 


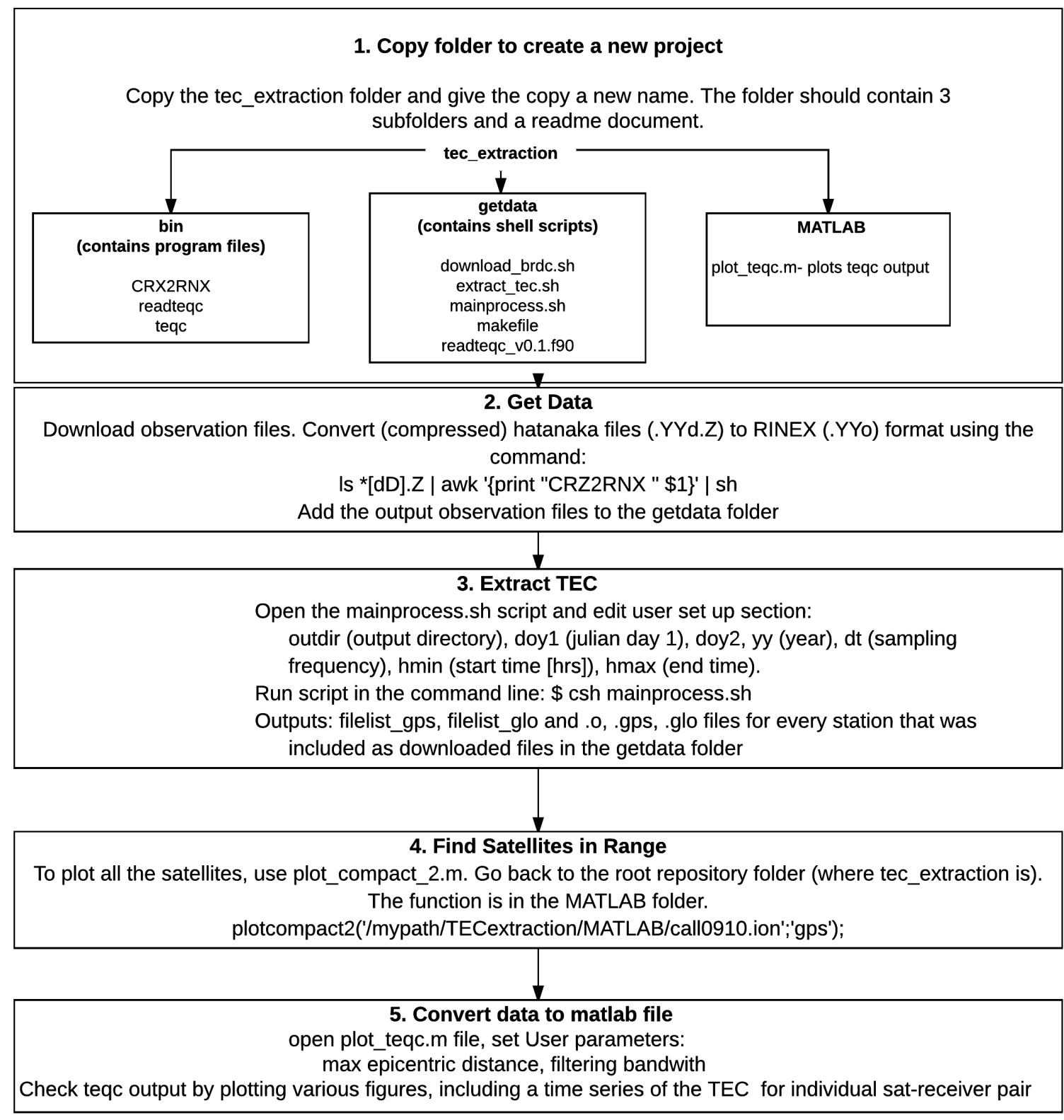

Figure 4.1: Workflow to extract TEC and convert to a MATLAB binary file. 

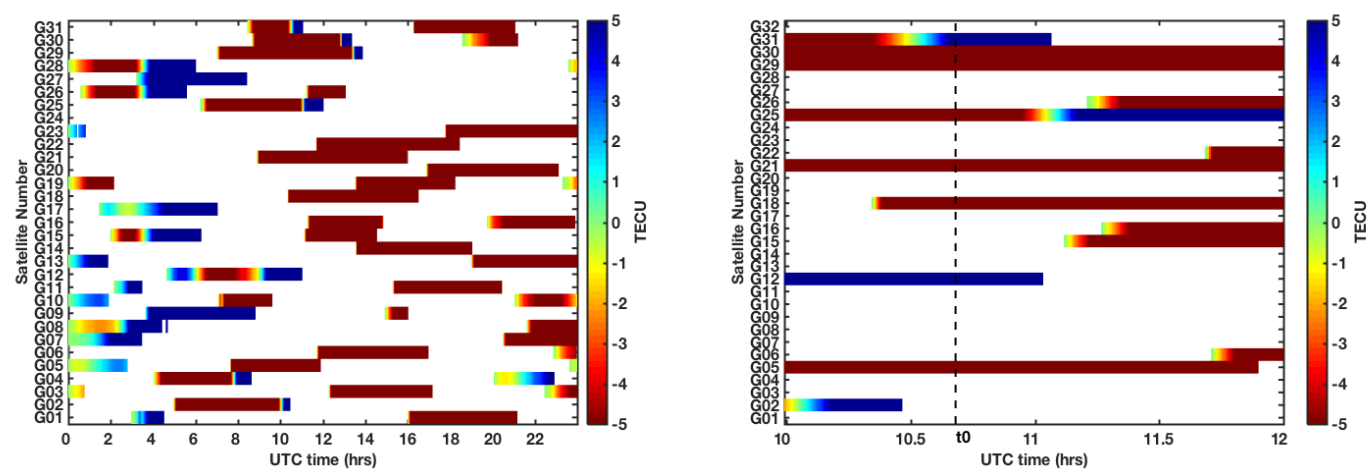

Figure 4.2: Example output from plot_compact_2.m. The plot on the left shows all satellites during a 24-hour time period. Satellites below the horizon have no data. The plot on the right displays times immediately before and after the 2011 Van (Turkey) earthquake. The black dashed line indicates the time of the earthquake.

http://terras.gsi.go.jp/ja/crx2rnx.html.

Teqc will extract the TEC when given two input files; the RINEX observation file and the RINEX navigation file. For more information on using teqc see Estey \& Meertens (1999).

\subsubsection{Workflow Example}

To automate the TEC extraction I use a workflow developed by Lucie Rolland (IPGP/LANL) and Virgile Rakoto (IPGP). This workflow is outlined in Figure (4.1). The workflow diagram is organized into five steps. Steps 1 through 3 walk through creating a new project folder, downloading the data and running teqc to extract the TEC. In step one, I show the contents for three folders I use for the TEC extraction. The bin folder contains program files for decompressing the Hatanaka files and the teqc program. In step two, I download the observation files and use the RNXCMP software program to decompress into RINEX format. In step three, I run a script called mainprocess.sh that downloads the navigation files and runs teqc to extract 


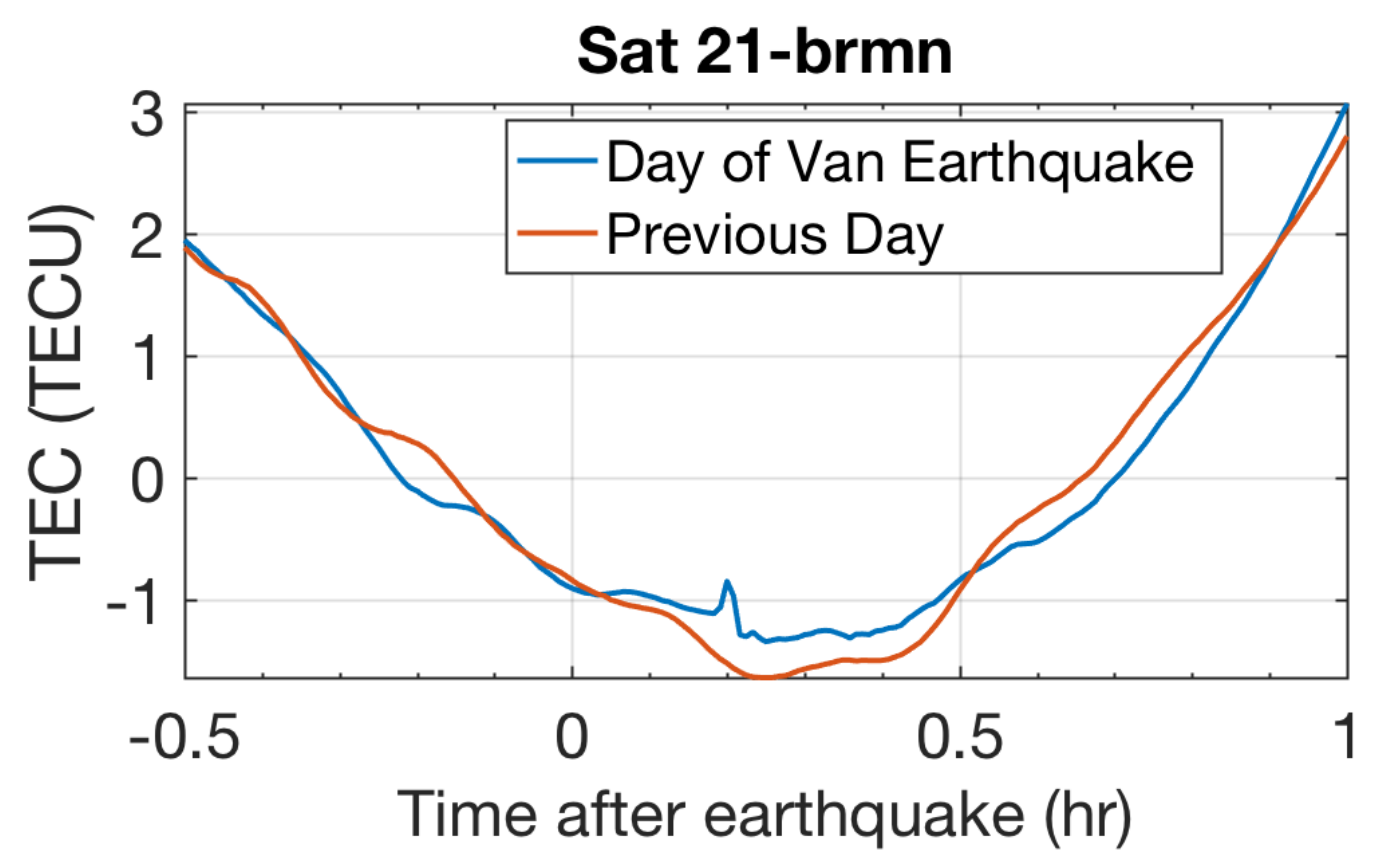

Figure 4.3: Plots of TEC time series for GPS satellite number 21 and station brmn (Turkey). Units are TEC units (TECU) where 1 TECU $=10^{16}$ electrons $/ \mathrm{m}^{2}$. The parabola shape is due to the motion of the satellite as the line-of-sight length through the ionosphere changes as the satellite orbits the Earth.

TEC.

Steps 4 and 5 use MATLAB scripts to plot all the data by satellite and then create a matlab file with the TEC data for a given time range. In the getdata folder I run the makefile to compile fortran code from readteqc_v0.1.f90. This creates a program to translate the teqc output to a matlab compatable format. In step 4, I look at data from all satellites to determine which satellites were in view of the receivers around the time of the earthquake or time of interest. Figure (4.2) shows an example of the output for the 2011 Van earthquake. The plot on the left shows the TEC for every satellite with the GPS satellite number plotted on the y axis and UTC on the $\mathrm{x}$ axis. Times with no data indicate that the satellite was below the horizon of the station. 
The plot on the right shows the same data zoomed in to times near the time of the Van earthquake at 10:41 UTC. By examining this figure, we see that satellites 5, 21, 25, 29 and 30 are in range before and after the earthquake. We can use this information to plot TEC for relevant satellites in step 5. Figure (4.3) shows an example of a time series of the TEC for station brmn in Turkey for the 2011 Van Earthquake. At about 12 minutes after the earthquake, there is a sudden change in the TEC that is not present in data from the day before. This initial check of the data quickly identifies possible pairs of satellites and receivers that show TEC disturbances.

\subsection{IPP Coordinates}

In Chapter 1, I described the IPP as the point of intersection between an ionospheric height and the line of sight of the satellite and receiver. The IPP is the point I use to assign a location to the TEC measurements. To obtain the IPP associated with my data, I use an International GNSS Service (IGS) product that contains high precision orbit positions within about a meter of accuracy (Dow et al., 2009). The IGS Rapid (IGR) product for the orbit positions are SP3 files and have the following format: igrwwwwd.sp3.Z, where wwww is the GPS week, $d$ is the day of week and Z indicates a compressed file. Since I know the location of the receiver, I can calculate the line of sight between the receiver and satellite for any time. I can then find the IPP for any height.

\subsubsection{Processing observed TEC}

In the next Chapter, I show TEC observations for the 2016 Kaikoura earthquake. After extracting the TEC, I apply a few processing steps to isolate the CID in the time series. I show an example of the processing in Figure 4.4. First, I apply a polynomial detrend of the data (middle plot). This removes the contribution of the 


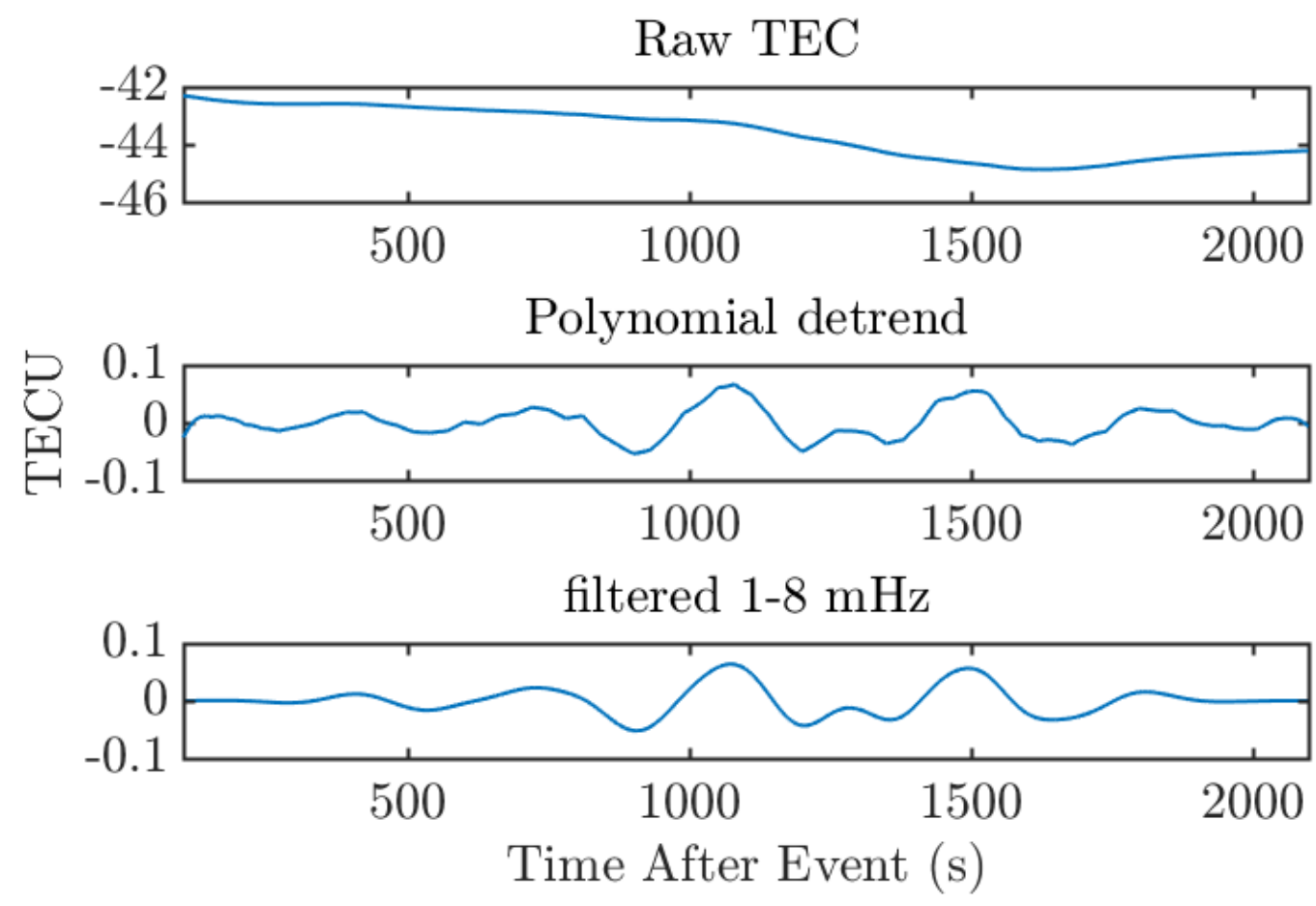

Figure 4.4: Example of processing for a single TEC time series from the 2016 Kaikoura event. The raw data (top), TEC after polynomial detrend (middle), final TEC after filtering (bottom)

satellite motion to the TEC. Then I taper the first and last $25 \%$ of the signal before applying a zero-phase, second-order FIR Butterworth filter from 1 to $8 \mathrm{mHz}$ (bottom plot). 


\title{
CHAPTER 5:
}

\section{TEC OBSERVATIONS AND MODELING OF THE 2016 KAIKOURA EARTHQUAKE}

\author{
Rebekah F Lee $^{1}$, Lucie M. Rolland ${ }^{2}$, T. Dylan Mikesell $^{1}$ \\ ${ }^{1}$ Environmental Seismology Laboratory, Department of Geosciences, Boise State \\ University, Boise, Idaho, USA \\ ${ }^{2}$ Géoazur, Université de Nice Sophia-Antipolis, UMR CNRS 7329, Observatoire de \\ la Côte dAzur, Valbonne, France
}

Submitted to Bulletin of the Seismological Society of America (BSSA), September 30,2017 


\subsection{Abstract}

We have processed GNSS time series data to extract total electron content (TEC) perturbations in the ionosphere due to the Kaikoura earthquake. We used ray-based modeling to infer which part of the Earth's surface coupled significant energy from the solid Earth into the atmosphere. We compared modeled TEC data to the observed time series data and determine that significant coupling occurred northeast of the initial slip. This work corroborates existing analysis made with geodetic and InSAR data. The TEC data suggested that the initial rupture coupled little energy into the atmosphere and only after significant surface displacements ( $\sim 60 \mathrm{~s}$ after the initiation) caused ionospheric perturbations. Using an array of GNSS stations, we were able to track the moveout of the acoustic wave through the ionosphere. We used a method commonly used in seismological studies called backprojection to estimate the exact location of the source of the TEC perturbation. This is the first time that this method has been applied to TEC data and the results are quite promising. The backprojection results are slightly shifted in space from the known area of maximum uplift, and we attribute this small discrepancy to the fact that we did not account for horizontal winds in the atmosphere in our travel-time modeling.

\subsection{Introduction}

The $\mathrm{M}_{\mathrm{w}}$ 7.8 November 13, 2016 Kaikoura earthquake resulted from a complex multifault rupture (Hamling et al., 2017) that initiated on a small strike-slip fault at 11:02:56 UTC beneath Waiau, in the North Canterbury region located in the centraleastern South Island of New Zealand. The rupture then propagated northeastward to trigger a large thrust-fault accounting for most of the energy release (Dupu- 
tel \& Rivera, 2017). The USGS estimated the earthquake epicenter location at $42.737^{\circ} \mathrm{S} / 173.054^{\circ} \mathrm{E}$ and $15.1 \mathrm{~km}$ depth. Severe ground shaking generated thousands of landslides with over 200 landslide dams created (Dellow et al., 2017). The largest of the landslides clustered around at least 21 faults that ruptured to the land or sea-floor surface. The Kaikoura earthquake complexity calls into question many conventional assumptions about the degree to which earthquake ruptures are controlled by fault segmentation and provides additional motivation to rethink these issues in seismic hazard models (Hamling et al., 2017).

Shallow earthquakes $(<\sim 30 \mathrm{~km})$ can excite infrasonic and acoustic-gravity waves that propagate in the fluid atmosphere layer of the Earth (e.g. Mutschlecner \& Whitaker, 2005). This happens because elastic waves and static displacements at the Earth's surface couple into acoustic-gravity waves in the atmosphere at very specific frequencies; these frequencies are related to specific modes of oscillation where the solid-fluid coupling is efficient (Lognonne et al., 1998). Acoustic-gravity wave frequencies are in the $\mathrm{mHz}$ range and, due to low attenuation in the atmosphere at these frequencies, can travel up to ionospheric altitudes where they can be detected using radio sounding. The ionosphere is the section of Earth's atmosphere that extends from $\sim 80 \mathrm{~km}$ above Earth's solid surface to more than $\sim 2000 \mathrm{~km}$ altitude, with the peak of ionization (i.e. electron density) occurring around 250-350 km altitude, depending on the location, day and time. When the acoustic-gravity waves interact with the ionosphere they perturb the ambient electron density (Georges, 1967; Hooke, 1970). These perturbations can be observed with Total Electron Content (TEC) recordings (e.g. Calais \& Minster, 1995; Afraimovich et al., 2001) derived from dual-frequency GNSS (Global Navigation Satellite System) data. TEC observations offer new insights 
into surface processes (e.g. uplift) during earthquake rupture (Rolland et al., 2013; Cahyadi \& Heki, 2014) and tsunami generation (Artru et al., 2005; Occhipinti et al., 2013). These signals can also offer insights into crustal structure via observations of Rayleigh wave propagation (Ducic et al., 2003; Occhipinti et al., 2010; Rolland et al., 2011; Reddy \& Seemala, 2015). Here we investigate the link between coseismic TEC perturbations and the 2016 Kaikoura earthquake surface motions.

In this article, we first review the geologic setting in which the Kaikoura event occurred, and review existing studies related to the surface ruptures and displacements that occurred as a result of slip. We discuss the data processing and modeling before presenting the observed TEC perturbations. The TEC data were recorded on numerous GNSS stations communicating with multiple satellites visible during the time of rupture. Using known information about the initiation time and event epicenter, we model the TEC perturbations and compare to the observed TEC perturbation. Finally, we use the seismic technique known as backprojection to locate at the surface of Earth where the solid-fluid coupling was largest. We interpret these results in the context of known rupture dynamics and post-rupture static displacements observed by other means.

\subsection{Geologic Setting and Surface Motion}

The Kaikoura earthquake occurred in a tectonically complex region between two subduction zones (Sti, 2017; Duputel \& Rivera, 2017). Along the North Island, the Pacific Plate subducts westward beneath the Australian Plate in the Hikurangi Subduction Zone; while to the south of New Zealand, the Australian Plate subducts beneath the Pacific Plate. The South Island of New Zealand sits in a zone of transpression between these two subduction zones. The Kaikoura earthquake occurred along the 
north end of this transition zone between the Alpine Fault, created by oblique continental collision, and the Marlborough Fault System and North Canterbury fault and fold domain (Sti, 2017). Figure 5.1 shows the tectonic setting in this section of New Zealand, in the vicinity of the Kaikoura rupture area. Although the epicenter was located within the North Canterbury fault domain, the main energy release was within the Marlborough Fault System (Hamling et al., 2017). Geodetic and geological field observations reveal surface ruptures along at least 12 major crustal faults and extensive uplift along much of the coastline. Surface displacements measured by GPS and satellite radar data show horizontal offsets of $\sim 6 \mathrm{~m}$ (Hamling et al., 2017). More relevant to the work presented here, a fault-bounded block (the Papatea block) was uplifted by up to $8 \mathrm{~m}$ (Hamling et al., 2017), while other areas of greater spatial extent to the northeast were uplifted by $\sim 4 \mathrm{~m}$.

\subsection{Methods}

We aggregate GNSS data from permanent ground-based GNSS receivers located across New Zealand. These data are derived from three networks: GNS, GeoNet and LINZ. We choose station-satellite pairs that offer ionospheric coverage within $1000 \mathrm{~km}$ of the epicenter. For each station-satellite pair that meets the observation criteria, we further process the GNSS data to extract the TEC signal. During the Kaikoura earthquake, five GPS satellites and one GLONASS satellite were visible across 184 GNSS receivers. In the remainder of this section we discuss the processing and modeling steps that enable the comparison of observed and synthetic TEC time series signals. 


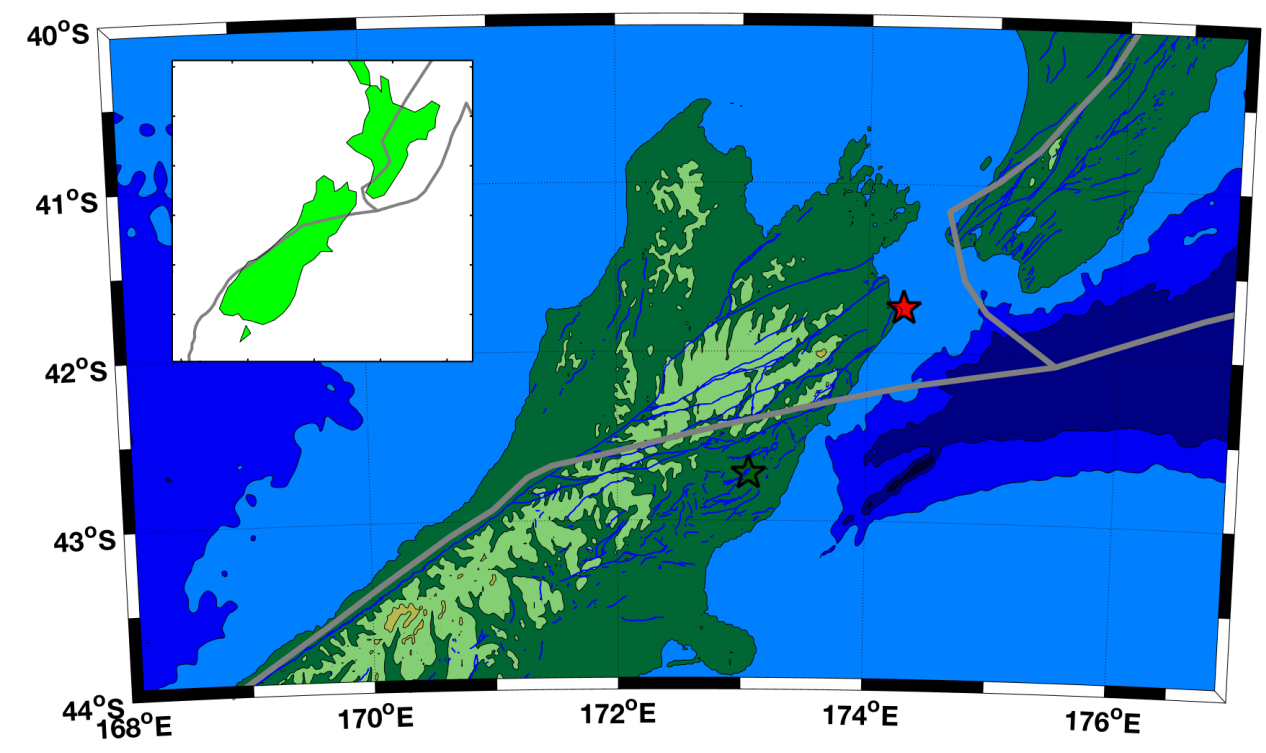

Figure 5.1: Tectonic setting and major faults in New Zealand across the northeast part of the southern island. The large southern star marks the Kaikoura earthquake epicenter estimated by USGS. The gray lines indicate plate boundaries, with other active faults as thin black lines. The star to the northeast of the southern island indicates the second location (Cape Campbell) where significant surface displacements occurred over a wide area (e.g. Hamling et al. (2017)). 


\subsubsection{IPP locations}

In order to map a TEC perturbation in space and time, we assume that the measurement is located at the intersection of the line-of-sight (LOS) between the GNSS receiver and the satellite and a thin shell located at $320 \mathrm{~km}$ altitude. This intersection is often referred to as the ionospheric pierce point (IPP). The IPP moves through space as the satellite travels in orbit. This assumption comes from the fact that the ionosphere has a peak in the electron density around an altitude of $320 \mathrm{~km}$ (as derived from IRI, the International Reference Ionosphere model (Bilitza \& Reinisch, 2008) in this region of the Southern Hemisphere at the time and location of the earthquake. A map of the GNSS stations locations is presented in Figure 5.2. This map also shows the location of IPP tracks through time for the six satellites visible to station WGTN (white triangle). The star indicates the start of the IPP track, and each track maps the IPP location for the 35 minutes following the initial rupture.

Satellites G20 and G29 each crossed the area with high elevation angles of $\sim 79^{\circ}$ and $\sim 74^{\circ}$, respectively. Thus they sound a shorter spatial extent of the ionosphere than the other satellites over the 35 minute time period. The IPPs for G20 move from the northwest to southeast, while G29 moves from the south to the north. Tracks of the IPPs for satellite G21 moved west to east and had a smaller elevation angle $\left(\sim 41^{\circ}\right)$, and lead to a longer track than the G20 and G29 tracks. Satellites G05 and G13 also have small elevation angles averaging $44^{\circ}$ and $51^{\circ}$, respectively. Aside from G21, all IPPs for WGTN locate to the east of the Kaikoura epicenter, including R22. We point out that as the elevation angle decreases, the accuracy of the thinlayer approximation and IPP location decreases and errors in the location of the TEC perturbation increase. We test the validity of the assumed IPP locations in the 


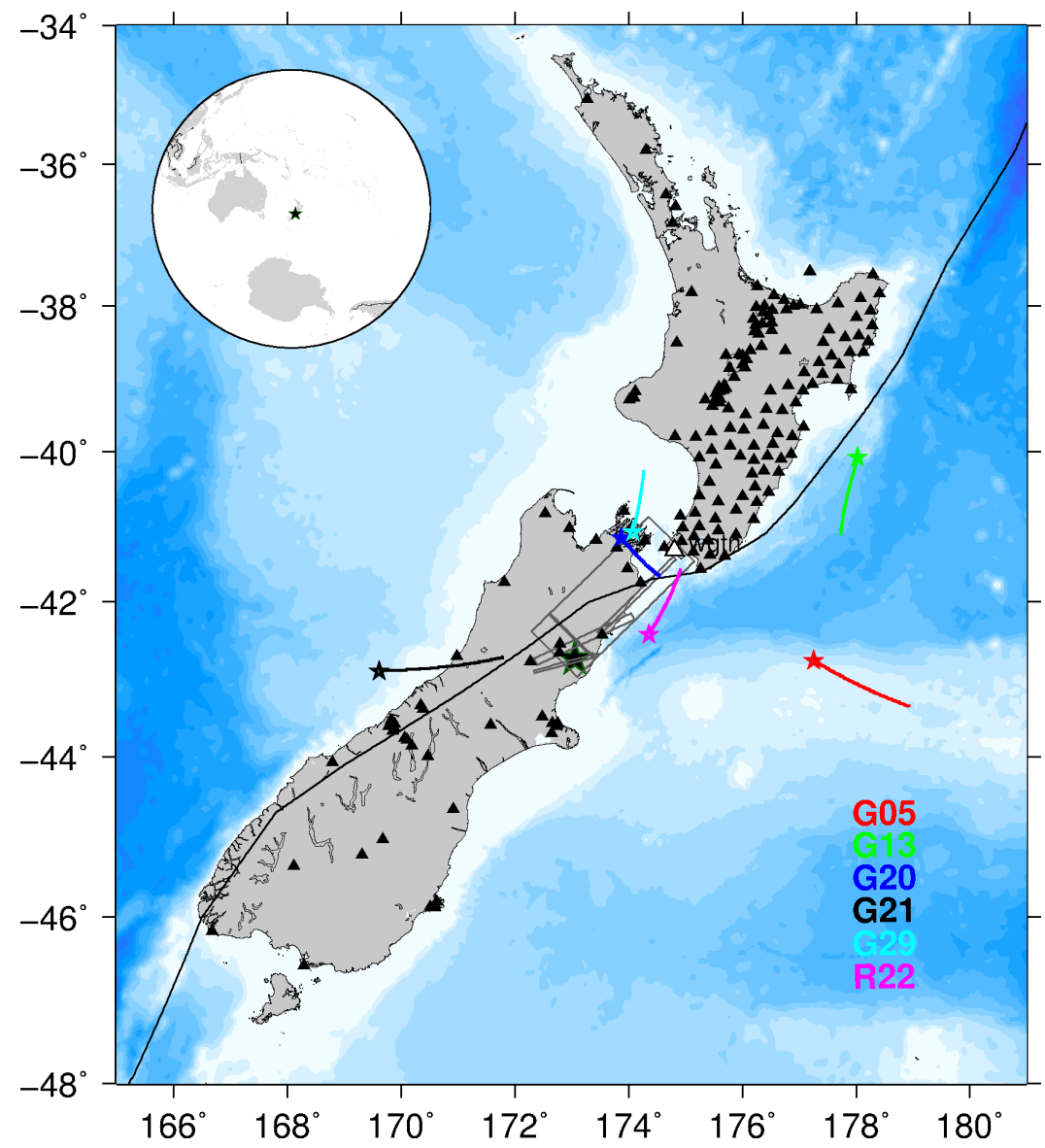

Figure 5.2: Location of GNSS stations with IPP tracks for the six satellites for station WGTN (white triangle). Stars for each IPP track correspond to the earthquake onset time. Large star on south island is the initial rupture location. Rectangles are fault planes projected to the surface.

modeling part of this study.

\subsubsection{TEC modeling}

The modeling approach follows three steps detailed by Rolland et al. (2013). First, we model the acoustic source as a purely compressional point source located at the Earth's surface and propagate the perturbation using a three-dimensional Hamiltonian acoustic ray tracing in a 1D (stratified) atmospheric model (Dessa et al., 
2005). Note that in this study, the anisotropic contribution of horizontal winds is not included. The solar radiation strongly impacts the atmosphere temperature and molecular composition used to derive parameters of the propagation medium (i.e. sound speed and density). Therefore, the 1D atmospheric model (Figure 5.3) used in the first step of the TEC modeling is computed at the location and time of the earthquake using the NRLMSISE model (Picone et al., 2002) with solar-radio flux at $10.7 \mathrm{~cm}$ (F107) parameter of 78 (moderate solar activity). Figure 5.3 shows the sound speed and amplification factor profiles with height. Due to conservation of kinetic energy, the wave amplitude increases as the inverse square root of the air density. This effect is counterbalanced by the viscous dissipation that increases as the density decrease. Figure 5.3 shows the modeled amplification factor of a $4 \mathrm{mHz}$ vertically propagating plane wave after Online et al. (1978). For the atmospheric conditions at the Kaikoura earthquake time and epicenter, this factor reaches $1.2 \times 10^{5}$ at 265 $\mathrm{km}$ of altitude then rapidly decreases. In the second step, the neutral atmosphere is coupled to the ionosphere to create the electron density perturbation caused by the interaction between the ionosphere, the acoustic wave, and geomagnetic field. In the final step, the TEC perturbation is computed as the integration of electron density perturbation along the satellite-station line-of-sight.

\subsubsection{TEC data extraction and processing}

We extract the biased slant TEC time series from the phase differences of the two carrier frequencies (Mannucci et al., 1998; Rolland et al., 2013). For each time series we apply a polynomial detrend to remove the satellite motion contribution and then taper the first and last $25 \%$ of the signal before applying a zero-phase second-order FIR Butterworth filter from 1 to $8 \mathrm{mHz}$. To highlight the satellite-station pairs that 

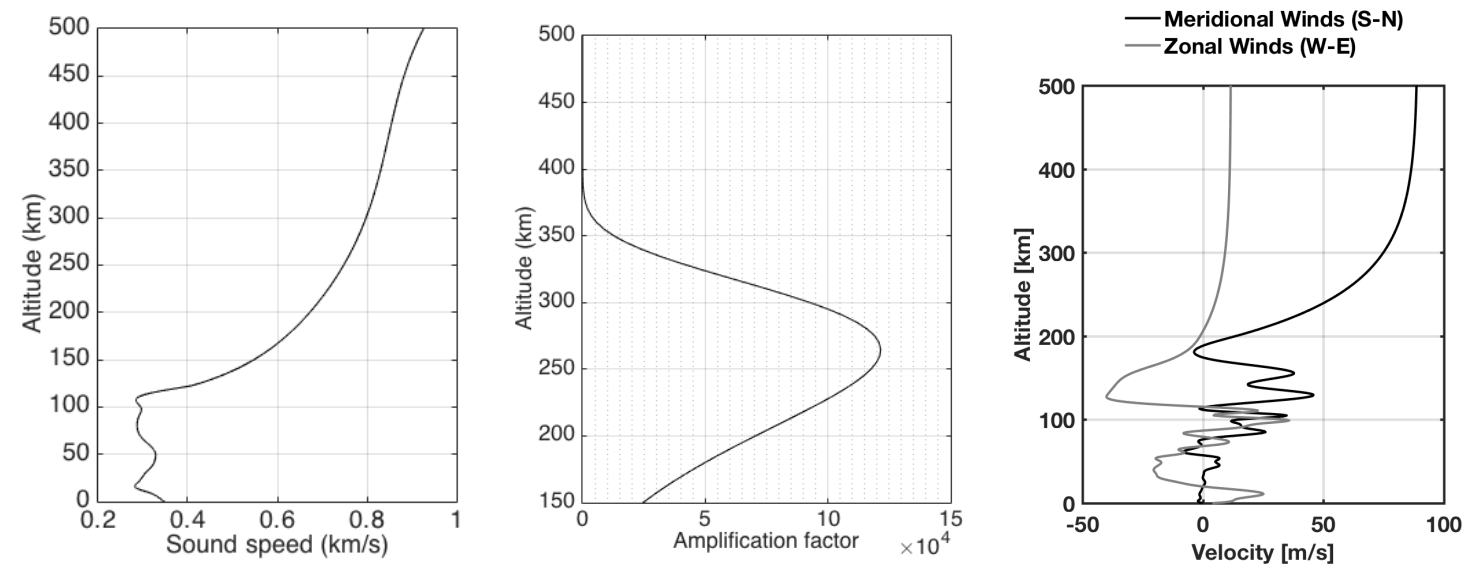

Figure 5.3: Left: Atmospheric sound speed profile computed from the NRLMSISE model (Picone et al., 2002) on November 13, 2016 above the rupture area. Center: Atmospheric amplification factor. Right: Horizontal wind model computed from the NRLHWM (Drob et al., 2015) on the same day above Epicenter 1.

better show the coseismic TID, we examine the signal-to-noise ratio (SNR) of each signal. We calculate the SNR as the maximum of the absolute value of the signal divided by the root-mean square of the signal. The SNR is always higher for the modeled TEC because natural background TEC fluctuations are not present.

\subsubsection{Backprojection}

Both dip-slip and strike-slip events are known to cause ionospheric perturbations related to Earth's surface motion near the epicenter (Astafyeva et al., 2014; Cahyadi \& Heki, 2014). The geomagnetic field plays a significant role in the amplitude and polarity of TEC recordings and care must be taken to discriminate the tectonic and non-tectonic contributions when characterizing the earthquake source. For example, Rolland et al. (2013) demonstrate that directivity in the TEC signal is related to the geometry of the line-of-sight between the satellite and receiver, rather than being caused by rupture geometry of the source. The amplitude of the observed TEC 
signal depends heavily on the geomagnetic latitude of the event, the GNSS receiver locations and the satellite look angle (Rolland et al., 2011; Cahyadi \& Heki, 2014). Up to now little work has gone into utilizing the TEC signal for earthquake source characterization. Acoustic source localization of the 1999 Chi-Chi earthquake $\left(\mathrm{M}_{w} 7.6\right)$ from coseismic ionospheric disturbances arrival times was proposed by Liu et al. (2010) and the impact of the 2004 Sumatra-Andaman earthquake $\left(\mathrm{M}_{w} 9.4\right)$ multiple ruptures on the observed GPS-derived TEC signals was investigated by Heki et al. (2006). In this paper we take a technique commonly applied in seismology and apply it to TEC recordings. Ishii et al. (2005) demonstrated the power of the back-projection method to understand where radiated energy comes from within the subsurface. Here we follow this idea and use back-projection in an attempt to determine where on the surface of the Earth these ionospheric perturbations originate.

We apply the stacked-power back-projection method (e.g. Haney, 2014) to the Kaikoura TEC data. In this implementation of back-projection, we compute the stacked power as

$$
p\left(\mathbf{r}_{o}\right)=\int_{0}^{t}\left(\frac{1}{N} \sum_{i=1}^{N} u_{i}\left(t+\tau_{s, i}-\tau_{o, i}\right)\right)^{2}+\left(\frac{1}{N} \sum_{i=1}^{N} H\left[u_{i}\left(t+\tau_{s, i}-\tau_{o, i}\right)\right]\right)^{2} d t
$$

where $u$ is the amplitude normalized TEC trace and $H$ is the Hilbert transform operator. The phase from the real source at station $i$ is represented by $\tau_{s, i}$, while the phase correction from a hypothetical source at image point $\mathbf{r}_{o}$ is represented as $\tau_{o, i}$. Here we take the phase correction term from the ray-theoretical arrival times discussed in the previous section and $N$ is the total number of station-satellite pairs. 


\subsection{Results}

To investigate the complexity of the event (i.e. extended and multiple fault ruptures), we consider two epicenter locations. The first is the location where the rupture initiated, taken from the USGS catalog at $42.72^{\circ} \mathrm{S}, 173.06^{\circ} \mathrm{E}$ (here after referred to as Epicenter 1). The second location (Epicenter 2) is Cape Campbell at $41.73^{\circ} \mathrm{S}$ and $174.28^{\circ} \mathrm{E}$. This second location is in the area of significant observed coastal uplift (Hamling et al., 2017). Source time functions for the Kaikoura event show a larger release of energy after / 60 seconds after the initial event (Duputel \& Rivera, 2017; Kaiser et al., 2017). Therefore, we model Epicenter 2 with an initial time 60s later

than Epicenter 1. We model the TEC considering an acoustic point source located at each of the two epicenters.

\subsubsection{TID from TEC signal}

To highlight the TID propagating radially from the epicenter, we plot the filtered TEC on a travel-time diagram, where the time-varying IPP location is used to compute the time-varying epicentral distance. This yields images similar to a seismic shot record, but with non-constant offset. We present travel-time diagrams for satellite G20 using Epicenter 1 (left panel) and Epicenter 2 (center panel) in Figure 5.4. For both epicenters, a distance-dependent moveout is observed. This moveout is directly related to the sound velocity in air, and the gray solid line indicates the apparent horizontal moveout for a wave traveling at $1 \mathrm{~km} / \mathrm{s}$. The zero-offset intercept on the time axis is shifted from zero to account for the propagation time of the acousticgravity wave from the surface to an altitude of $320 \mathrm{~km}$ (the assumed altitude of IPPs). We analyze the moveout to find a best fit velocity of $884 \mathrm{~m} / \mathrm{s}$ in Appendix B for satellite G20. Travel-time diagrams for other satellites are available in Appendix 

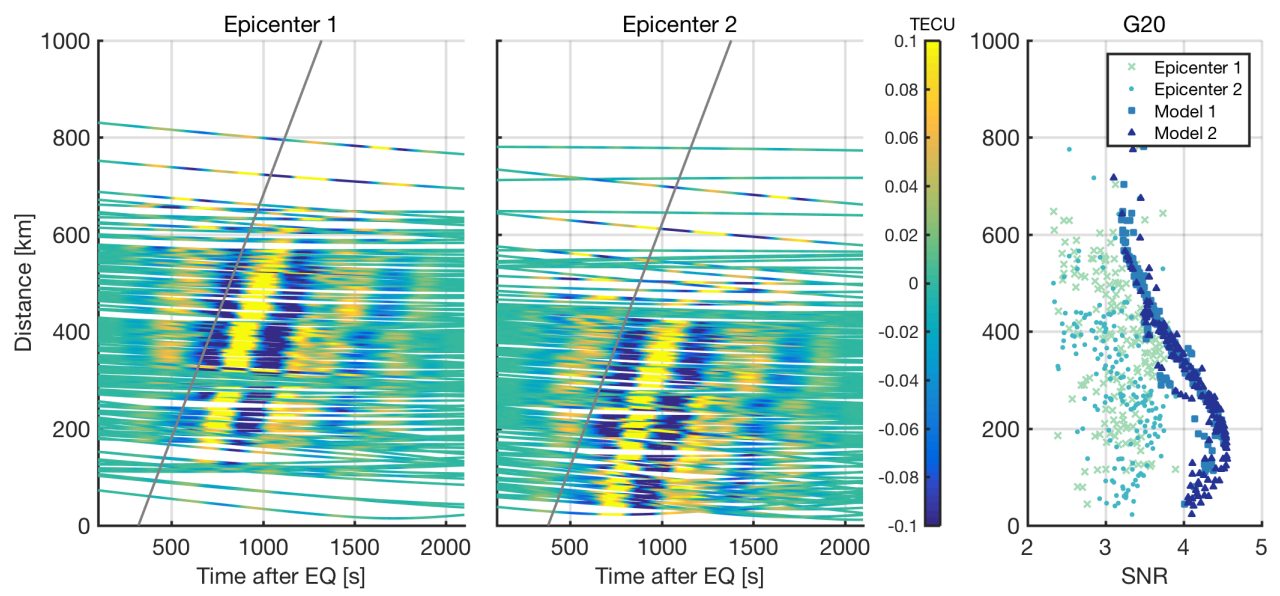

Figure 5.4: TEC observations from all stations plotted as a function of time and epicentral distance. Distance is calculated from the IPP to two potential epicenters: (left) Epicenter $\mathbf{1}$ at $42.72^{\circ} \mathrm{S} 173.06^{\circ} \mathbf{E}$ and (middle) Epicenter 2 at $41.73^{\circ} \mathrm{S}, 174.28^{\circ}$. Grayscale (color online) indicates the amplitude of the TEC in TEC units (TECU), where 1 TECU $=1 \times 10^{16}$ electrons per meter squared. The gray line marks the expected arrival time of an acoustic wave traveling radially at $1 \mathrm{~km} / \mathrm{s}$ from the chosen epicenter and the $\mathrm{x}$-intercept corresponds the time for the wave to propagate from the surface to an altitude of $320 \mathrm{~km}$. Right: signal-to-noise ratio of observed ( $\mathrm{x}$ and dots) and modeled (squares and triangles) TEC plotted by epicentral distance of the IPP at the time of maximum value of observed TEC for Epicenter 1 and Epicenter 2.

D. In most cases, the perturbations align in phase with the $1 \mathrm{~km}$ moveout, indicating the perturbations propagate radially from the epicenter area. Better alignment of the moveout for Epicenter 2 (Figure 5.5 and Figures S1-S3, available in the electronic supplement to this article) suggests this area contributes the most to the TEC signal. Interestingly, for satellite G21 the perturbations align better to Epicenter 1 than Epicenter 2 (Figure S2, available in the electronic supplement to this article). This suggests that satellite G21 observations are more sensitive to Epicenter 1 than to Epicenter 2, or the accuracy of the IPP locations for this satellite are low.

We examine the SNR of the modeled and observed TEC (right panel, Figure 5.4). 
We present both the observed ( $\mathrm{x}$ and dots) and modeled (squares and triangles) SNR for Epicenter 1 and Epicenter 2, respectively. We plot the SNR vs. epicentral distance, where distance is computed from the epicenter to the IPP at the time of the maximum absolute value of the observed TEC. In order to eliminate low quality data we use a quality metric derived from the observed data.

Due to the geometry of the geomagnetic field, the acoustic wave vector, and the satellite-station line-of-sight, some TEC perturbation amplitudes are weak (e.g. $\sim 300 \mathrm{~km}$ epicentral distance in Figure 5.4). To highlight the satellite-station pairs that better show the coseismic TID, we remove signals where the maximum observed TEC is below a threshold of 0.05 TECU. We present the high-quality stations for satellite G20 in Figure 5.5. The observed TID is now more coherent, and this method to select stations eliminates the observations with no clear signal; however, it becomes more difficult to differentiate between signal and background noise at far distances due to low SNR.

We also note that we have removed some of the scatter in the SNR of the observed TEC. With only the SNR for the selected stations plotted in the right panel, it is more apparent that using Epicenter 2 places the SNR of the observed TEC at distances resembling the shape of the SNR curve for the modeled TEC.

We present a comparison of the modeled and observed TEC time series for G20 and R22. We choose high quality stations (see Figure 5.5 for G20 and Figure S1 for R22, available in the electronic supplement to this article) that are common to both model results, and are from a range of distances and azimuths to both epicenters. We show the location of the stations selected for both satellites in Figure 5.6 as well as their respective IPPs. G20 and R22 sample similar areas of the ionosphere, but have 

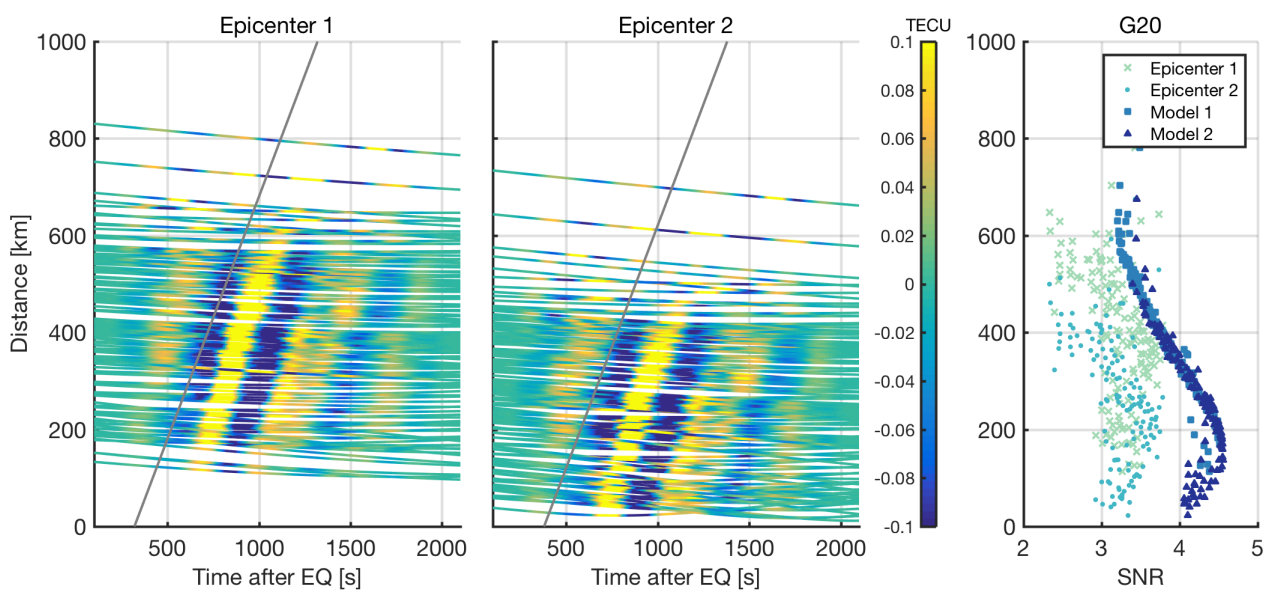

Figure 5.5: Stations with maximum amplitude $\geq 0.05$ TECU from those in Figure 5.4 for Epicenter 1 (left) and Epicenter 2 (middle). Right: SNR as in Figure 5.4 for selected stations

different geometries between the line-of-sight (LOS) and waves propagating from the rupture area. This is important for the amplitude of the observed TID.

Figure 5.7 displays the timeseries of selected stations for the two satellites. The maximum observed TEC is given on the right of each panel. G20 shows higher amplitudes (max of 0.33 TECU vs 0.09 ) and we were able to identify high quality stations at further distances (stations aukt and ktia). This is caused by the geometry of the LOS. Heki et al. (2006) and Rolland et al. (2011) illustrated that satellite station pairs with a LOS parallel or antiparallel to the direction of propagation will show little response to the event because the phase constructs negatively along the LOS, while a perpendicular geometry gives maximum response. G20 has a LOS nearly perpendicular to waves propagating from the rupture area and therefore captures the response of the TEC, while R22 crosses the propagating wave at a shallow angle, producing a smaller amplitude in TEC perturbations.

For both satellites, Model 2 predicts arrival times more accurately the Model 1. 


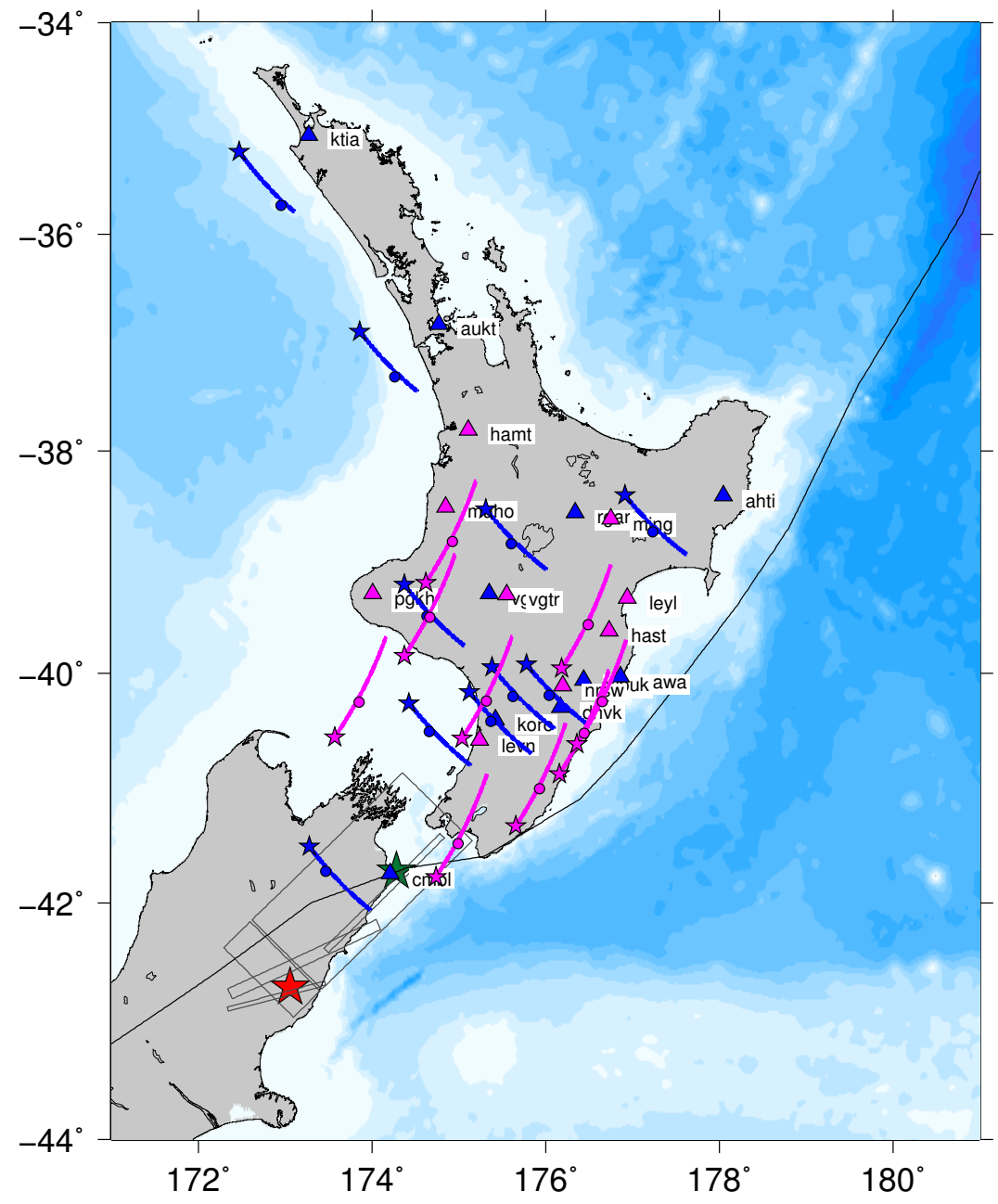

Figure 5.6: Map of the IPPs selected for the modeling study. Curves show the IPPs trajectories at $320 \mathrm{~km}$ height for LOS linking GNSS stations marked as triangles to satellites G20 (northeast to south east tracks) and R22 (southwest to northeast tracks). The same time window as in Figure 5.2 is used. Additionally, the small stars mark the IPP locations at the earthquake onset time. The disks show the location of IPP when the observed TEC perturbation is maximum. Note that due to the GNSS satellites orbital motion, the IPP trajectories of satellite G20 (respectively R22) here describe a southwestward (respectively southward) translation of the selected network, moving southeastward (respectively northward) with time. The large stars mark the epicenter locations chosen as inputs to models 1 and 2 . 

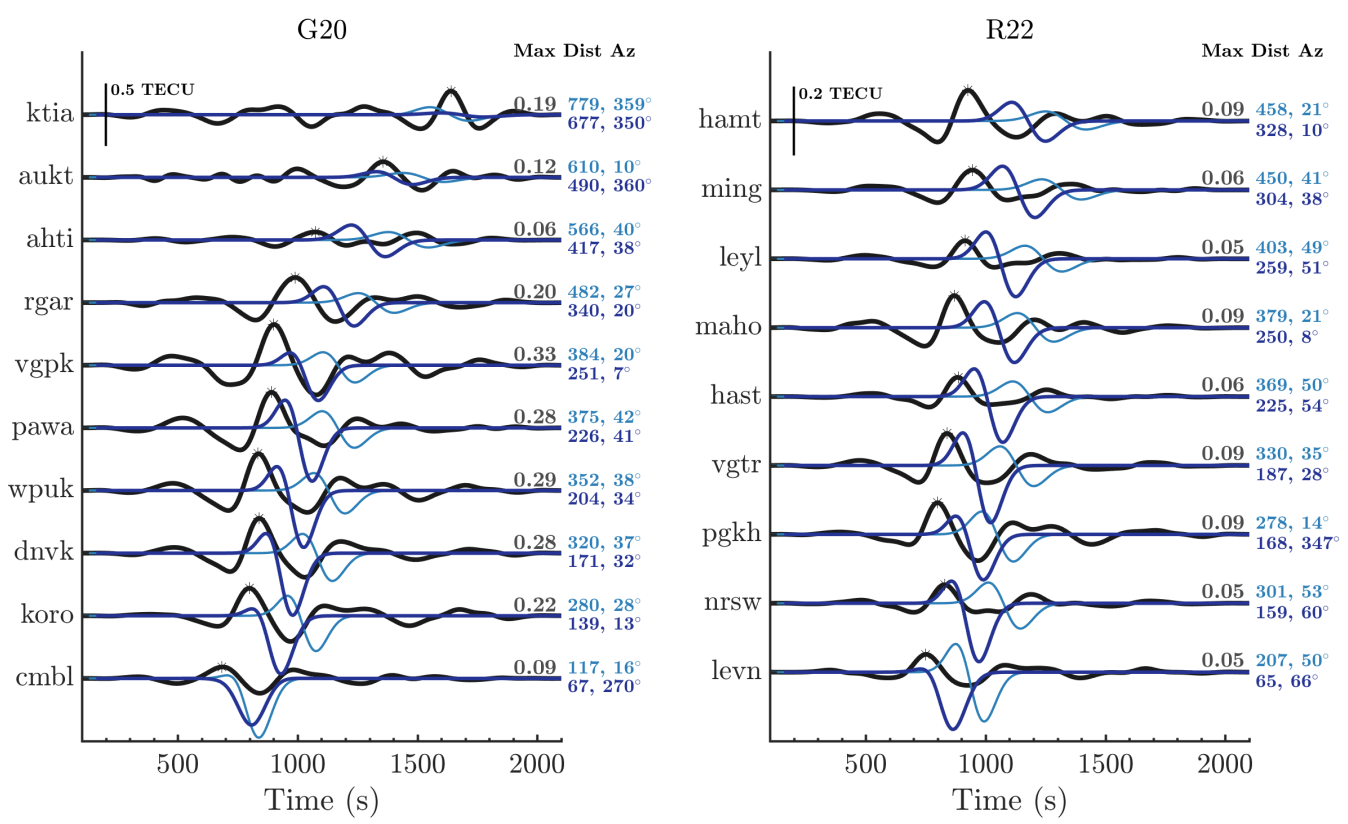

Figure 5.7: Comparison of observed TEC time series (black, thick line) and modeled TEC using Epicenter 1 (light, thin line) and Epicenter 2 (medium dark line). Time is in reference to the initialization of the Kaikoura rupture and correspondence to time zero for Model 1. Model 2 is shifted 60 seconds to account for the difference in start time. Maximum observed TEC is shown at right, along with the distance from each epicenter to the station and the azimuth of the IPP at the time of maximum TEC. We indicate the maximum TEC time by a black dot for each observed TEC series. Stations are plotted in order of distance to the Epicenter 2 (medium dark line). The scale for the $y$-axis of each station is shown on the top stations. 
For satellite G22 stations nrsw, hast, and leyl have the best fit and are all within $50^{\circ}-60^{\circ}$ azimuth to Epicenter 2. For G20, stations dnvk and pawa showed the best

fit. Delay between Model 2 and observed data increases with distance. This could be attributed to the wind which was moving northward at $75 \mathrm{~m} / \mathrm{s}$ at $300 \mathrm{~km}$ (Figure 5.3). Since the IPPs are all to north this could impact the arrival time significantly at greater distances. In nearly all cases except when the IPPs fall between the two epicenters (station cmbl, G20) Model 1 does not fit the data in either arrival time or phase. This observation and the better alignment with an event at Epicenter 2 indicates that significant coupling occurred northeast of the initial rupture.

\subsubsection{Backprojection Results}

Before applying the backprojection to the observed data, we first applied backprojection to the synthetic data. Due to the fact that the backprojection relies on phase shifts and interference patterns across the array, initial phase of the TEC is important. Gõmez et al. (2015) demonstrated that the phase shift caused by the coupling into the ionosphere and the LOS integration cause the TEC phase to vary dramatically from the acoustic wave phase. Thus due to the geometry of the system when TEC is measured, the phase can be different from the acoustic wave. This has the potential to blur or completely change the backprojection results when using TEC signals rather than acoustic or seismic waves as done in solid Earth studies.

Using a plane-wave approximation and far-field simplifications, Gõmez et al. (2015) computed the phase shift analytically and then deconvolved the TEC signal to recover the acoustic wave prior to beamforming analysis. Gõmez et al. (2015) neglected amplitude effects and focused only on phase. In a similar manner, we normalize the TEC traces prior to backprojection; however we do not deconvolve the TEC signal 

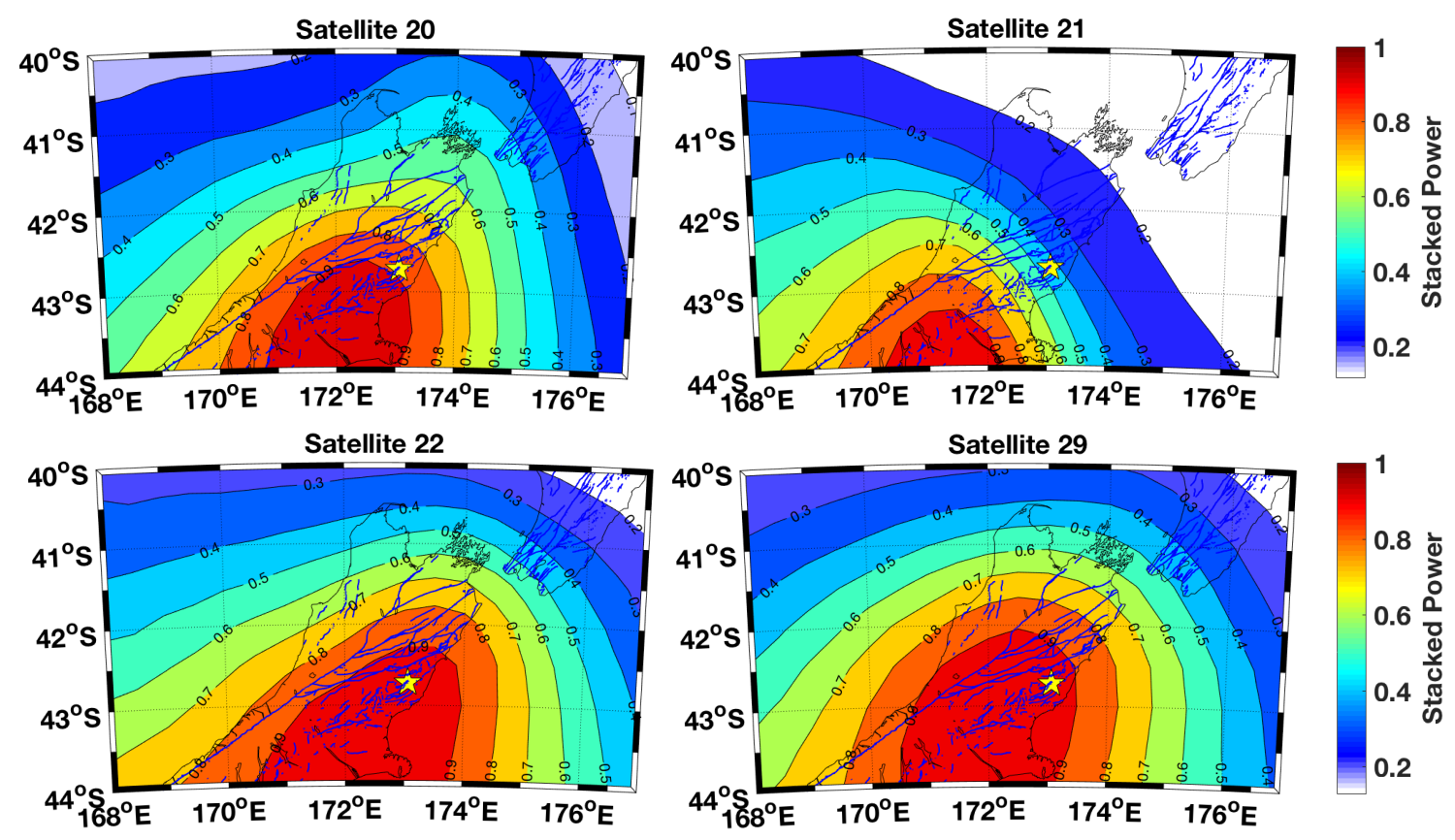

Figure 5.8: Backprojection results from TEC synthetic time series for the Epicenter 1 model (large star). Most station and IPP locations lie to the northeast of the epicenter and have varying IPP tracks (e.g. station WGTN in Figure 5.2).

to estimate the acoustic signal. We are unable to do that in this instance because we are not dealing with plane waves. The results of the backprojection from synthetic data modeled for a source at Epicenter 1 are presented in Figure 5.8. We used only the data that passed the quality control (e.g. left panel in Figure 5.5) and had an IPP epicentral distance less than $600 \mathrm{~km}$.

All four backprojection images show smearing along a line trending southwest to northeast. This is a direct result of the lack of resolution caused by the array being largely located to the northeast of the epicenter. Another interesting observation is that the two satellites that are moving from the southwest to the northeast (R22 and G29) result in backprojection maximum near the epicenter, while the other two 

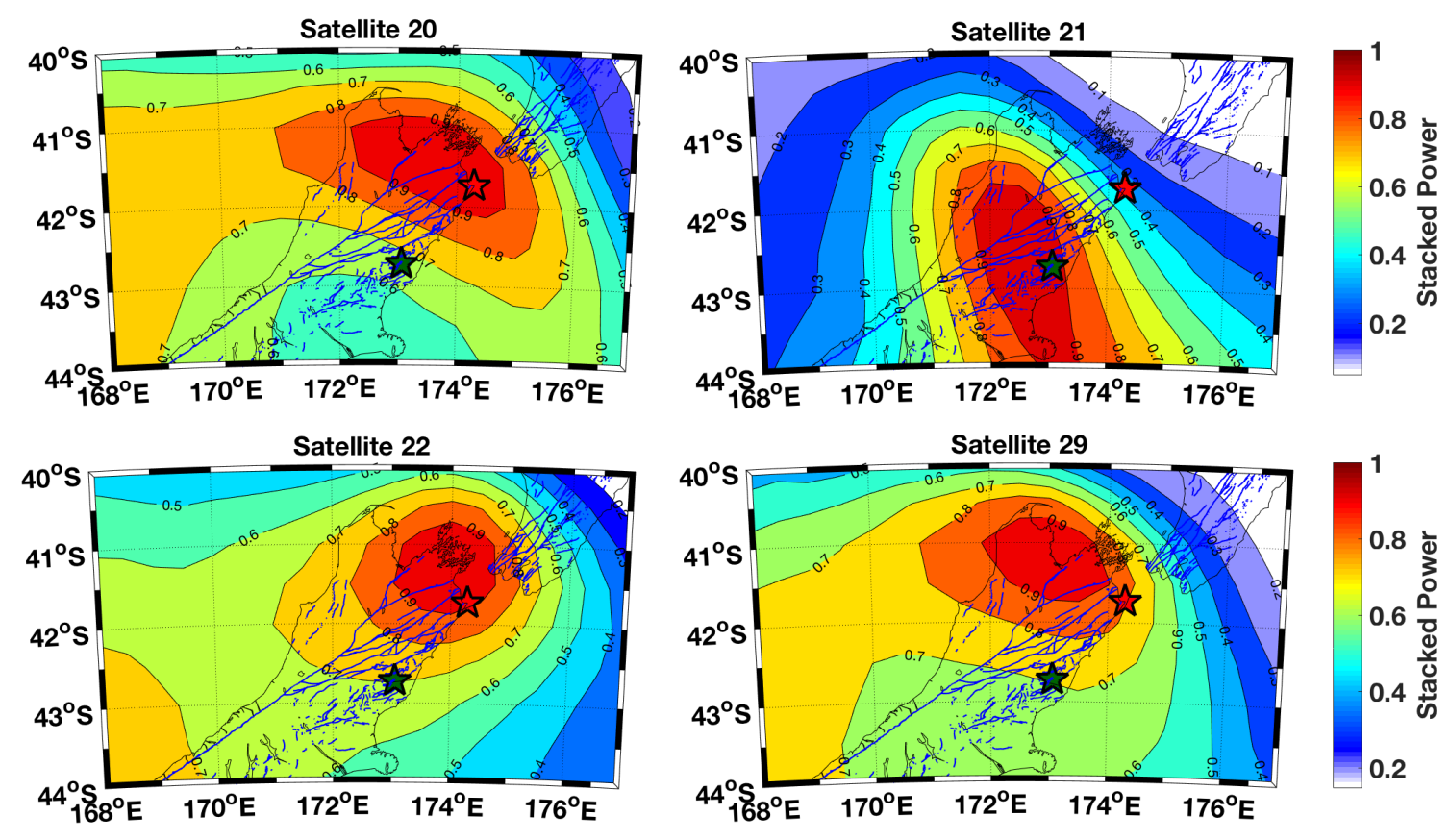

Figure 5.9: Backprojection results from observed TEC time series. The geographic grid used here is $0.5 \times 0.5$ degrees.

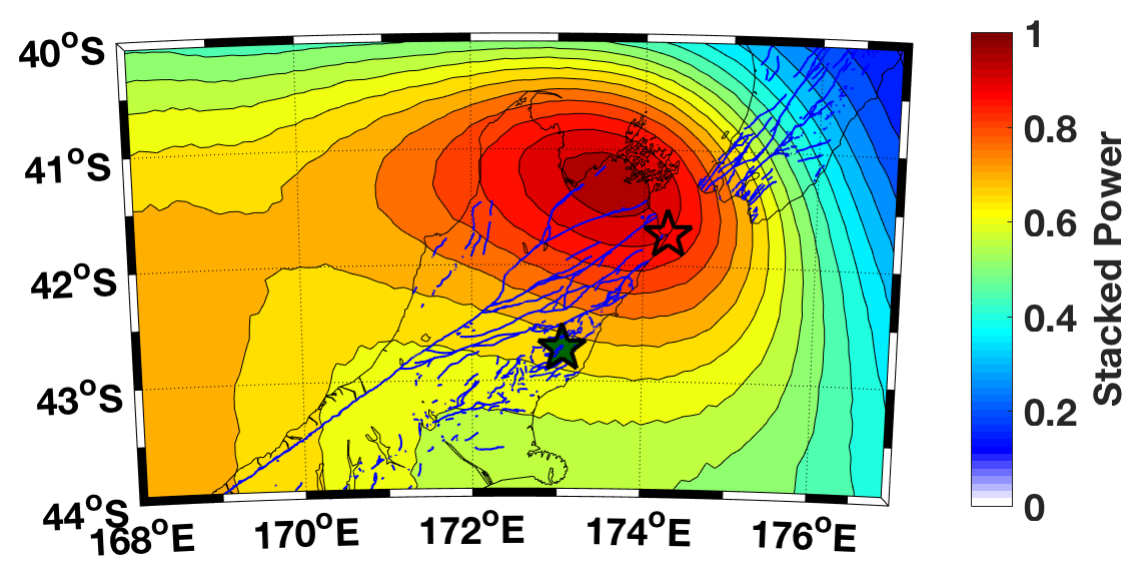

Figure 5.10: Averaged backprojection results from satellites G20, R22 and G29 in Figure 5.9. The geographic grid used here is $0.1 \times 0.1$ degrees. 
satellites (G20 and G21) do not. We attribute this to the TEC phase shifts mentioned above. In the latter case, the LOS integration and the directional differences between the acoustic wave-vector and the magnetic field lead to a phase bias that is not compensated for or corrected by the backprojection. The backprojection works by applying a phase shift based on the modeled acoustic wave traveltimes. Therefore, we deduce that until the TEC phase can be corrected to match the incident acoustic field, the backprojection may have limited utility for TEC studies. That said, we can still investigate the backprojection results of satellites R22 and G29 from the observed data.

We present the backprojection from the individual satellites in Figure 5.9. As expected, the results vary quite significantly from satellite to satellite. Considering that each satellite-station pair is sensing a different area of the ionosphere, we can average these backprojection images to try to suppress artifacts due to the different IPP array geometries and highlight areas of the backprojection image that are consistent. The average backprojection images from satellites G20, R22 and G29 is presented in Figure 5.10. Based on the results from the synthetic tests, we choose to leave out satellite G21 from the average. Looking at the average, we observe a rather large, but well defined source region. The location of this source region is to the northwest of the area identified in previous studies (e.g. Hamling et al. (2017)) as the region of maximum surface displacement.

\subsection{Discussion}

Clear coseismic ionospheric signatures linked to the Kaikoura earthquake was observed by 4 satellites out of 6 satellites in visibility. The first disturbance was detected around 540 seconds after the earthquake initiation by the low elevation satellite G21 
that sensed the ionosphere right above the rupture area. Interestingly, the signature of Rayleigh surface waves in the ionosphere was not observed in the analyzed dataset. A refined analysis using high-rate (1 sps) data should be conducted to better sense those fast $(\sim 3.5 \mathrm{~km} / \mathrm{s})$ propagating disturbances. Travel time diagrams all show an ionospheric signature consistent with the direct acoustic wave impulsed by the Earth's surface motions and propagating with an apparent horizontal velocity of $\sim 1 \mathrm{~km} / \mathrm{s}$. The largest amplitudes were observed North of the rupture area, which is typical for earthquakes located at mid-latitude in the southern hemisphere. All these features are successfully reproduced by TEC modeling.

Discrepancies between the observed and modeled data can be attributed to a number of approximations made in the modeling. First, we assume a single point source as for the case of the 2011 Van earthquake of magnitude $\mathrm{M}_{w} 7.3$ where the single N-shape signature was successfully modeled by Rolland et al. (2013). This assumption is not as accurate here as we can see in the observed data: a distorted N-shape signature is observed (e.g. Figure 5.5) and even some time series (ahti-G20, vgpk-G20, satellite G21, available in the electronic supplement to this article) show a secondary N-shape wave signature. A more refined analysis should be conducted with the support of modeling in order to investigate the possibility of discriminating the relative contributions of multiple sources.

In the work we presented here, we also make assumptions about the IPP locations and we find that this assumption is critical for the backprojection approach. First, we assume the IPP height is the same for all satellite-stations pairs and located at the maximum of ionization height estimated by an empirical model (i.e. IPP $=320 \mathrm{~km}$ ). Besides, our model also shows that the neutral wave amplitude is maximum at around 
$265 \mathrm{~km}$ height, suggesting that an intermediate altitude would be more representative of the IPP height. Moreover, the ionosphere can have significant topography, which would cause the maximum in the electron density to vary in altitude. Because the satellite is moving through space, the IPP height should change with time. Due to this assumption we can expect to see some variations in arrival times and amplitudes between the observed and modeled data. This is a topic of ongoing research.

Regarding the location of the source estimated in the average backprojection, at this point we can attribute the discrepancy in location relative to either epicenter to a few things. First, the difference in phase between the acoustic wave and the TEC time series. Second, the lack of complete IPP coverage in the atmosphere. Array geometry is known to cause artifacts in backprojection methods (e.g. Haney (2014)). Third, our use of a 1D layered atmospheric model could be leading to bias in the results. We favor the third potential cause. We currently estimate travel times with a ray-tracing that does not incorporate the winds in the atmosphere. However, considering the horizontal wind model at this location (right panel in Figure 5.3), we note that winds may play a role. The dominant wind vector during the earthquake is to the northwest according the NRLHWM (Drob et al., 2015). For wind speeds of $25-50 \mathrm{~m} / \mathrm{s}$, an acoustic wave traveling for $\sim 1000$ s could propagate 10s of kilometers in the direction of the wind. Thus wind leads to wave propagation phenomena not accounted for in the travel-time calculation. In future work, we will incorporate horizontal winds into the travel-time calculation. We hypothesize that this would move the source location to the southeast in the average backprojection image and improve the correlation between known surface displacements and source regions estimated by backprojection. 


\subsection{Conclusion}

We demonstrate that the Kaikoura earthquake created significant ionospheric perturbations in the electron density. This is due to coupling of energy at the Earth's surface into the atmosphere. This energy propagates to ionospheric heights where the acoustic wave interacts with the ionosphere. Using GNSS data recorded by stations across New Zealand, we extracted the total electron content signal from six satellites. Many satellite-station pairs show identifiable TIDs, with a moveout velocity of approximately $1 \mathrm{~km} / \mathrm{s}$, indicating a propagating acoustic wave. Using a point source approximation, we model the full 3D atmosphere-ionosphere interaction to create synthetic TEC time series. Using these synthetic data we test two potential locations for impulsive sources. We find that the second source, closely associated with subsurface sources from other studies that occurred $\sim 60 \mathrm{~s}$ after the initial rupture, give the closest waveform match. This is because the location of the initial rupture did not cause the significant surface displacements needed to couple significant energy into the atmosphere.

To further confirm this result, we applied the backprojection technique to focus the energy observed in the time series back to the location of origin. The backprojection results averaged over all satellites indicate a location to the northwest of the proposed epicenter we chose from modeling. We attribute this discrepancy to not accounting for horizontal winds in the atmosphere during travel-time contributions for the backprojection. This lack of wind in the modeling is a potential source of misfit in the waveform comparison as well. This is the first time the backprojection method has been performed using TEC data. The results are promising and methods to account for phase differences between acoustic and TEC time series are needed, 
as well as methods to improve focusing of the backprojection. The dominant TEC is $4 \mathrm{mHz}$, which controls the resolution achievable in the backprojection images and eventually limits the ability of the method to resolve two sources close to each other in space. Satellites that have low elevation angles lead to poorly recovered TEC signals, as observed here and predicted by theory. Large elevation angles lead to IPPs directly above the source region and provide high-quality data for backprojection and potentially other source related studies. Finally, observations of TEC combined with accurate backprojection may in the future prove useful for subduction earthquakes that happen far from the coast under water. These types of events cannot be directly sensed by geodetic methods such as classical geodetic positioning and InSAR; however, onland GNSS stations sensing the ionosphere directly above offshore subduction events could provide an image of the rupture area. This type of information is critical for tsunami hazard and monitoring. 


\section{CHAPTER 6:}

\section{CONCLUSIONS}

Obtaining the acoustic wave from TEC measurements would open more research opporunities for understanding the characteristics of TID sources on the surface. In natural hazards, this would give responders more information on what is happening on the ground in the cases of large earthquakes and tsunamis.

This work shows that at least in some cases, we may be able to approximate the ionosphere as varying only in the vertical direction. However, the factors influencing the importance of a 3D ionospheric model are complex and, until these factors are better understood, routine use of the analytical model to obtain a transfer function should be postponed.

Future investigation of the analytical model should control satellite-receiver LOS and examine the relationship of the coupling factor with the waveform differences from 1D and 3D ionospheric divergences. This analysis should be conducted for a range of times throughout the year and for different latitudes. Insight into the cut-off for elevation angle would provide additional important constraints on the applicability of the analytical model.

Until investigations similar to those described above are complete, the numerical model with 3D ionospheric divergence will provide the most reliable results. The numerical implementation would be as follows: 
1. Create an impulsive acoustic wave to find the ionospheric impulse response/transfer function.

2. Test the accuracy of the transfer function by inverting results from the full model of the N-shaped acoustic wave with the transfer function. This should produce the original acoustic wave.

3. Invert actual TEC data using the transfer function to obtain the acoustic wave.

As this model relies on a compressional point source, it would be best applied to ruptures with simple source-time functions.

In Chapter 5, we give an example of applying seismic methods to TEC. We apply backprojection directly on the TEC and find promising results. Location discrepancies could be explained by local winds as the error was consistent with wind speed and direction, but we also note the need to account for the phases differences between the acoustic wave and TEC. Inverting for the acoustic wave is one promising way of accomplishing this. Backprojection using inverted acoustic time series should improve localization results. We also note that the coupling frequency of the acoustic wave limits the maximum resolution from backprojection images in the case of two sources close to each other. This is because of the long wavelength of the acoustic wave.

While elevation angle above the cut-off did not influence results of modeling between 1D and 3D ionospheric modeling, it is important that the IPP tracks be directly above the source area. For subduction zone events far from the coast, TEC observations, as well as seismic techniques such as backprojection, can provide important information for tsunamis hazard and modeling. In these cases, GNSS stations sensing the ionosphere directly above the offshore event could supplement geodetic and 
InSAR observations that are limited by the distance of receivers to the source area. 


\section{REFERENCES}

2017. The Mw7.8 2016 Kaikura Earthquake: Surface Fault Rupture and Seismic Hazard Context. Bulletin of the New Zealand Society for Earthquake Engineering, $\mathbf{5 0}(2), 73-84$.

Afraimovich, E. L., Ding, Feng, Kiryushkin, V. V., Astafyeva, E. I., Jin, Shuanggen, \& Sankov, V. a. 2010. TEC response to the 2008 Wenchuan Earthquake in comparison with other strong earthquakes. International Journal of Remote Sensing, 31(13), $3601-3613$.

Afraimovich, Edward L, Perevalova, Natalia P, Plotnikov, a. V, \& Uralov, a. M. 2001. The Shock-Acoustic Waves Generated by Earthquakes. Annales Geophysicae, $\mathbf{1 9}(4), 395-409$.

Afraimovich, Edward L., Astafyeva, Elvira I., Demyanov, Vladislav V., Edemskiy, Ilya K., Gavrilyuk, Nadezhda S., Ishin, Artem B., Kosogorov, Eugene A., Leonovich, Lyudmila A., Lesyuta, Oleg S., Palamartchouk, Kirill S., Perevalova, Natalia P., Polyakova, Anna S., Smolkov, Gennadyi Y., Voeykov, Sergey V., Yasyukevich, Yury V., \& Zhivetiev, Ilya V. 2013. A Review of GPS/GLONASS Studies of the Ionospheric Response to Natural and Anthropogenic Processes and Phenomena. Journal of Space Weather and Space Climate, 3(aug), A27.

Artru, Juliette, Ducic, Vesna, Kanamori, Hiroo, Lognonné, Philippe, \& Murakami, 
Makoto. 2005. Ionospheric Detection of Gravity Waves Induced by Tsunamis. Geophysical Journal International, 160, 840-848.

Astafyeva, Elvira, \& Heki, Kosuke. 2009. Dependence of Waveform of Near-Field Co seismic Ionospheric Disturbances on Focal Mechanisms. 939-943.

Astafyeva, Elvira, Rolland, Lucie M, \& Sladen, Anthony. 2014. Strike-Slip Earthquakes Can Also Be Detected in the Ionosphere. Earth and Planetary Science Letters, 405, 180-193.

Beer, Tom. 1974. Atmospheric Waves. New York: John Wiley \& Sons.

Bilitza, D, \& Reinisch, B W. 2008. International Reference Ionosphere 2007: Improvements and New Parameters. Advances in Space Research, 42(4), 599-609.

Cahyadi, M N, \& Heki, K. 2014. Coseismic Ionospheric Disturbance of the Large Strike-Slip Earthquakes in North Sumatra in 2012: $M_{w}$ Dependence of the Disturbance Amplitudes. Geophysical Journal International, 200, 116-129.

Calais, Eric, \& Minster, J Bernard. 1995. GPS Detection of Ionospheric Perturbations Following the January 17, 1994, Northridge Earthquake. Geophysical Research Letters, 22(9), 1045-1048.

Chum, J, Cabrera, M A, Mosna, Z, Fagre, M, Base, J, \& Fiser, J. 2016. Nonlinear Acoustic Waves in the Viscous Thermosphere and Ionosphere Above Earthquake. Journal of Geophysical Research: Space Physics, 121(12), 126-137.

Dautermann, Thomas, Calais, Eric, \& Mattioli, Glen S. 2009. Global Positioning System Detection and Energy Estimation of the Ionospheric Wave Caused by the 
13 July 2003 Explosion of the Soufrière Hills Volcano, Montserrat. Journal of Geophysical Research, 114(B2), B02202.

Davies, John B., \& Archambeau, Charles B. 1996. Modeling of Atmospheric and Ionospheric Disturbances from Shallow Seismic Sources.

Dellow, S., Massey, C., Cox, S., Archibald, G., Begg, J., Bruce, Z., Carey, J., Davidson, J., Della Pasqua, F., Glassey, P., Hill, M., Jones, K., Lyndsell, B., Lukovic, B., McColl, S., Rattenbury, M., Read, S., Rosser, B., Singeisen, C., Townsend, D., Villamor, P., Villeneuve, M., Godt, J., Jibson, R., Allstadt, K., Rengers, F., Wartman, J., Rathje, E., Sitar, N., Adda, A.-Z., Manousakis, J., \& Little, M. 2017. Landslides Caused by the $\mathrm{M}<\mathrm{inf}>\mathrm{w}</$ inf $>7.8$ Kaikura earthquake and the immediate response. Bulletin of the New Zealand Society for Earthquake Engineering, $50(2)$.

Dessa, J. X., Virieux, J., \& Lambotte, Sophie. 2005. Infrasound Modeling in a Spherical Heterogeneous Atmosphere. Geophysical Research Letters, 32(12), 1-5.

Dow, John M., Neilan, R. E., \& Rizos, C. 2009. The International GNSS Service in a Changing Landscape of Global Navigation Satellite Systems. Journal of Geodesy, 83(3-4), 191-198.

Drob, Douglas P., Emmert, John T., Meriwether, John W., Makela, Jonathan J., Doornbos, Eelco, Conde, Mark, Hernandez, Gonzalo, Noto, John, Zawdie, Katherine a., McDonald, Sarah E., Huba, Joe D., \& Klenzing, Jeff H. 2015. An Update to the Horizontal Wind Model (HWM): The Quiet Time Thermosphere. Earth and Space Science, 2(7), n/a-n/a. 
Ducic, V, Artru, J, \& Lognonné, P. 2003. Ionospheric Remote Sensing of the Denali Earthquake Rayleigh Surface Waves. Geophysical Research Letters, 30(18), 1951.

Duputel, Z., \& Rivera, L. 2017. Long-Period Analysis of the 2016 Kaikoura Earthquake. Physics of the Earth and Planetary Interiors, 265(apr), 62-66.

Estey, Louis H., \& Meertens, Charles M. 1999. TEQC: The Multi-Purpose Toolkit for GPS/GLONASS Data. GPS Solutions, 3(1), 42-49.

Georges, T. M. 1967. Evidence for the Influence of Atmospheric Waves on Ionospheric Motions. Journal of Geophysical Research, 72(1), 422.

Georges, T. M. 1968. Collisional Interaction of Atmospheric Waves With the Ionospheric F Region. Pages 377-380 of: Acoustic-Gravity Waves in the Atmosphere Symposium Proceedings.

Georges, T. M., \& Hooke, W. H. 1970. Wave-Induced Fluctuations in Ionospheric Electron Content: A Model Indicating Some Observational Biases. Journal of Geophysical Research, 75(31), 6295-6308.

Gõmez, Demián, Smalley, Robert, Langston, Charles A., Wilson, Terry J., Bevis, Michael, Dalziel, Ian W. D., Kendrick, Eric C., Konfal, Stephanie A., Willis, Michael J., Piñon, Diego A., Cimbaro, Sergio R., Caccamise, Dana, Gómez, Demián, Smalley, Robert, Langston, Charles A., Wilson, Terry J., Bevis, Michael, Dalziel, Ian W. D., Kendrick, Eric C., Konfal, Stephanie A., Willis, Michael J., Piñón, Diego A., Cimbaro, Sergio R., \& Caccamise, Dana. 2015. Virtual Array Beamforming of GPS TEC Observations of Coseismic Ionospheric Disturbances 
Near the Geomagnetic South Pole Triggered by Teleseismic Megathrusts. Journal of Geophysical Research A: Space Physics, 120(10), 9087-9101.

Grawe, Matthew A, \& Makela, Jonathan J. 2015. The Ionospheric Responses to the 2011 Tohoku , 2012 Haida Gwaii , and 2010 Chile Tsunamis: Effects of Tsunami Orientation and Observation Geometry.

Hamling, Ian J., Hreinsdóttir, Sigrún, Clark, Kate, Elliott, John, Liang, Cunren, Fielding, Eric, Litchfield, Nicola, Villamor, Pilar, Wallace, Laura, Wright, Tim J., D'Anastasio, Elisabetta, Bannister, Stephen, Burbidge, David, Denys, Paul, Gentle, Paula, Howarth, Jamie, Mueller, Christof, Palmer, Neville, Pearson, Chris, Power, William, Barnes, Philip, Barrell, David J. A., Van Dissen, Russ, Langridge, Robert, Little, Tim, Nicol, Andrew, Pettinga, Jarg, Rowland, Julie, \& Stirling, Mark. 2017. Complex Multifault Rupture During the 2016 M_w 7.8 Kaikura Earthquake, New Zealand. Science, 356(6334), 7194.

Haney, Matthew M. 2014. Backprojection of Volcanic Tremor. Geophysical Research Letters, 41(6), 1923-1928.

Heki, K, \& Ping, J. 2005. Directivity and Apparent Velocity of the Coseismic Ionospheric Disturbances Observed With a Dense GPS Array. Earth and Planetary Science Letters, 236(3-4), 845-855.

Heki, K, Otsuka, Y, Choosakul, N, Hemmakorn, N, Komolmis, T, \& Maruyama, T. 2006. Detection of Ruptures of Andaman Fault Segments in the 2004 Great Sumatra Earthquake With Coseismic Ionospheric Disturbances. Journal of Geophysical Research, 111(B09313). 
Hooke, William H. 1970. The Ionospheric Response to Internal Gravity waves: 1. The F2 Region Response. Journal of Geophysical Research, 75(28), 5535-5544.

Ishii, Miaki, Shearer, Peter M, Houston, Heidi, \& Vidale, John E. 2005. Extent, Duration and Speed of the 2004 Sumatra-Andaman Earthquake Imaged by the Hi-Net Array. Nature, 435(June), 933-936.

Kaiser, A, Balfour, N, Fry, B, Holden, C, Litchfield, N, Gerstenberger, M, D’Anastasio, E, Horspool, N, McVerry, G, Ristau, J, Bannister, S, Christophersen, A, Clark, K, Power, W, Rhoades, D, Massey, C, Hamling, I, Wallace, L, Mountjoy, J, Kaneko, Y, Benites, R, Van Houtte, C, Dellow, S, Wotherspoon, L, Elwood, K, \& Gledhill, K. 2017. The 2016 Kaikoura, New Zealand, Earthquake: Preliminary Seismological Report. Seismological Research Letters, 88(3), 727-739.

Kherani, E. A., Lognonné, P., Hébert, H., Rolland, L., Astafyeva, E., Occhipinti, G., Coïsson, P., Walwer, D., \& de Paula, E. R. 2012. Modelling of the Total Electronic Content and Magnetic Field Anomalies Generated by the 2011 Tohoku-Oki Tsunami and Associated Acoustic-Gravity Waves. Geophysical Journal International, 191(3), 1049-1066.

Liu, J Y, Tsai, H F, Lin, C H, Kamogawa, M, Chen, Y I, Lin, C H, Huang, B S, Yu, S B, \& Yeh, Y H. 2010. Coseismic Ionospheric Disturbances Triggered by the ChiChi Earthquake. Journal of Geophysical Research: Space Physics, 115(A08303).

Lognonne, P, Clevede, E, \& Kanamori, H. 1998. Computation of Seismograms and Atmospheric Oscillations by Normal-Mode Summation for a Spherical Earth Model With Realistic Atmosphere. Geophysical Journal International, 135(2), 388-406. 
Mannucci, a. J, Wilson, B D, Yuan, D N, Ho, C H, Lindqwister, U J, \& Runge, T F. 1998. A Global Mapping Technique for GPS-Derived Ionospheric Total Electron Content Measurements. Radio Science, 33(3), 565-582.

Mutschlecner, J. Paul, \& Whitaker, Rodney W. 2005. Infrasound from Earthquakes. Journal of Geophysical Research D: Atmospheres, 110(1), 1-11.

Occhipinti, Giovanni, Dorey, Philippe, Farges, Thomas, \& Lognonné, Philippe. 2010. Nostradamus: The Radar that Wanted to be a Seismometer. Geophysical Research Letters, $\mathbf{3 7}(\mathrm{sep}), \mathrm{L} 18104$.

Occhipinti, Giovanni, Rolland, Lucie M, Lognonné, Philippe, \& Watada, Shingo. 2013. From Sumatra 2004 to Tohoku-Oki 2011: The Systematic GPS Detection of the Ionospheric Signature Induced by Tsunamigenic Earthquakes. Journal of Geophysical Research: Space Physics, 118(L18104), 1-11.

Online, Cambridge Books, Nov, Mon, \& Raupach, M R. 1978. Fluid Mechanics. By L. D. Landau and E. M. Lifshitz. Translated from Russian by J. B. Sykes and W. H. Reid. Pergamon Press (Oxford, New York, Toronto, Sydney, Paris, Braunschweig), 1959 (reprinted 1975). Pp. xii, 536. £8.25. Quarterly Journal of the Royal Meteorological Society, 104(440), 535.

Pichon, Alexis Le, Blanc, Elisabeth, \& Hauchecorne, Alain. 2009. Infrasound Monitoring for Atmospheric Studies. Dordrecht: Springer Netherlands.

Picone, J. M., Hedin, A. E., Drob, D. P., \& Aikin, A.C. 2002. NRLMSISE-00 Empirical Model of the Atmosphere: Statistical Comparisons and Scientific Issues. Journal of Geophysical Research, 107(A12), 1468. 
Reddy, C. D., \& Seemala, Gopi K. 2015. Two-Mode Ionospheric Response and Rayleigh Wave Group Velocity Distribution Reckoned From GPS Measurement Following M_w 7.8 Nepal Earthquake on 25 April 2015. Journal of Geophysical Research: Space Physics, 120(8), 7049-7059.

Rolland, Lucie M, Lognonné, Philippe, \& Munekane, Hiroshi. 2011. Detection and Modeling of Rayleigh Wave Induced Patterns in the Ionosphere. Journal of Geophysical Research, 116(A05320).

Rolland, Lucie M, Vergnolle, Mathilde, Nocquet, Jean-mathieu, Sladen, Anthony, Dessa, Jean-xavier, Tavakoli, Farokh, Nankali, Hamid Reza, \& Cappa, Frédéric. 2013. Discriminating the Tectonic and Non-Tectonic Contributions in the Ionospheric Signature of the 2011, $M_{w}$ 7.1, Dip-Slip Van Earthquake, Eastern Turkey. Geophysical Research Letters, 40(11), 2518-2522.

Schunk, Robert W, \& Nagy, Andrew F. 2000. Ionospheres. Cambridge: Cambridge Univ. Press.

University of Alaska Fairbanks Geophysical Institute. 2017. The Importance of Ionospheric Research.

Warshaw, S.I., \& Dubois, P.F. 1981. Preliminary Theoretical Acoustic and RF Sounding Calculations for Mill Race. UCID 19231. 
APPENDIX A:

MISFIT ANALYSIS 
Here I show results of misfit analysis for 2 satellites paired with stations near the 2011 Van (Turkey) earthquake. I follow the same steps outlined in Chapter 3 to calculate the modeled TEC with a $1 \mathrm{D}$ and $3 \mathrm{D}$ ionosphere assumption. In each Figure, I show the raw amplitude time series (left panel) for each station assuming a 1D (black) and 3D (red) ionosphere. The results show a change in both amplitude and in phase between the 1D and 3D models. In the right panel, I show the amplitude normalized envelopes of the signals. I also give the root-mean-square (RMS) error for both the raw waveforms and the envelopes. This error is a combination of both phase and amplitude. The RMS error of the envelopes is lower than the raw waveforms (up to half), indicating that energy is conserved but that the phase is significantly different. 

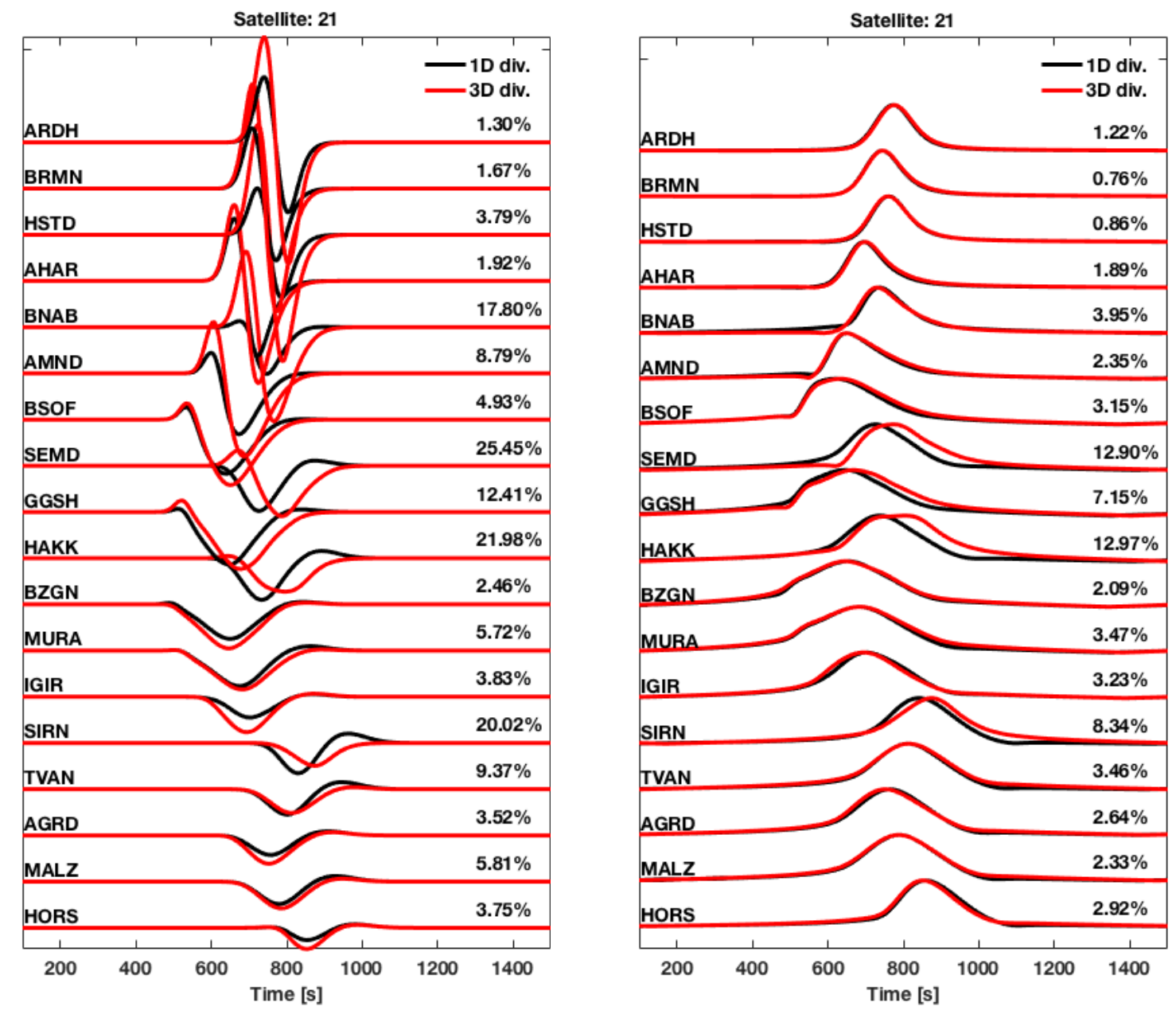

Figure A.1: Comparison of 1D and 3D TEC results. (Left panel) Raw waveforms and RMS error for stations near Van, Turkey paired with satellite 21. (Right Panel) Amplitude normalized envelope of the raw waveforms and corresponding RMS error. 

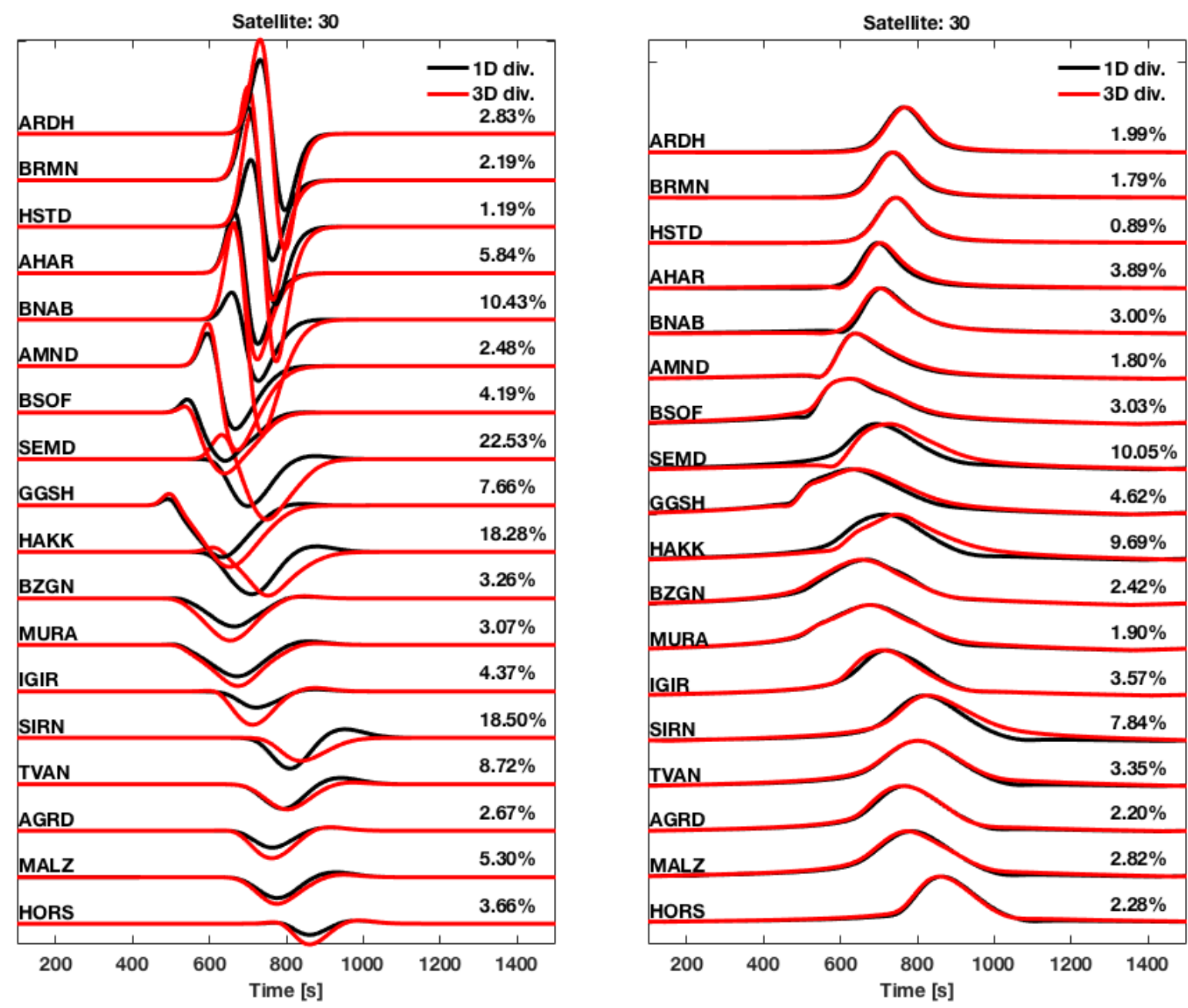

Figure A.2: Same as A.1 but for satellite 30. 
APPENDIX B:

\section{VELOCITY ANALYSIS}


Here we show velocity analysis for satellite G20. We find the best fit line to the

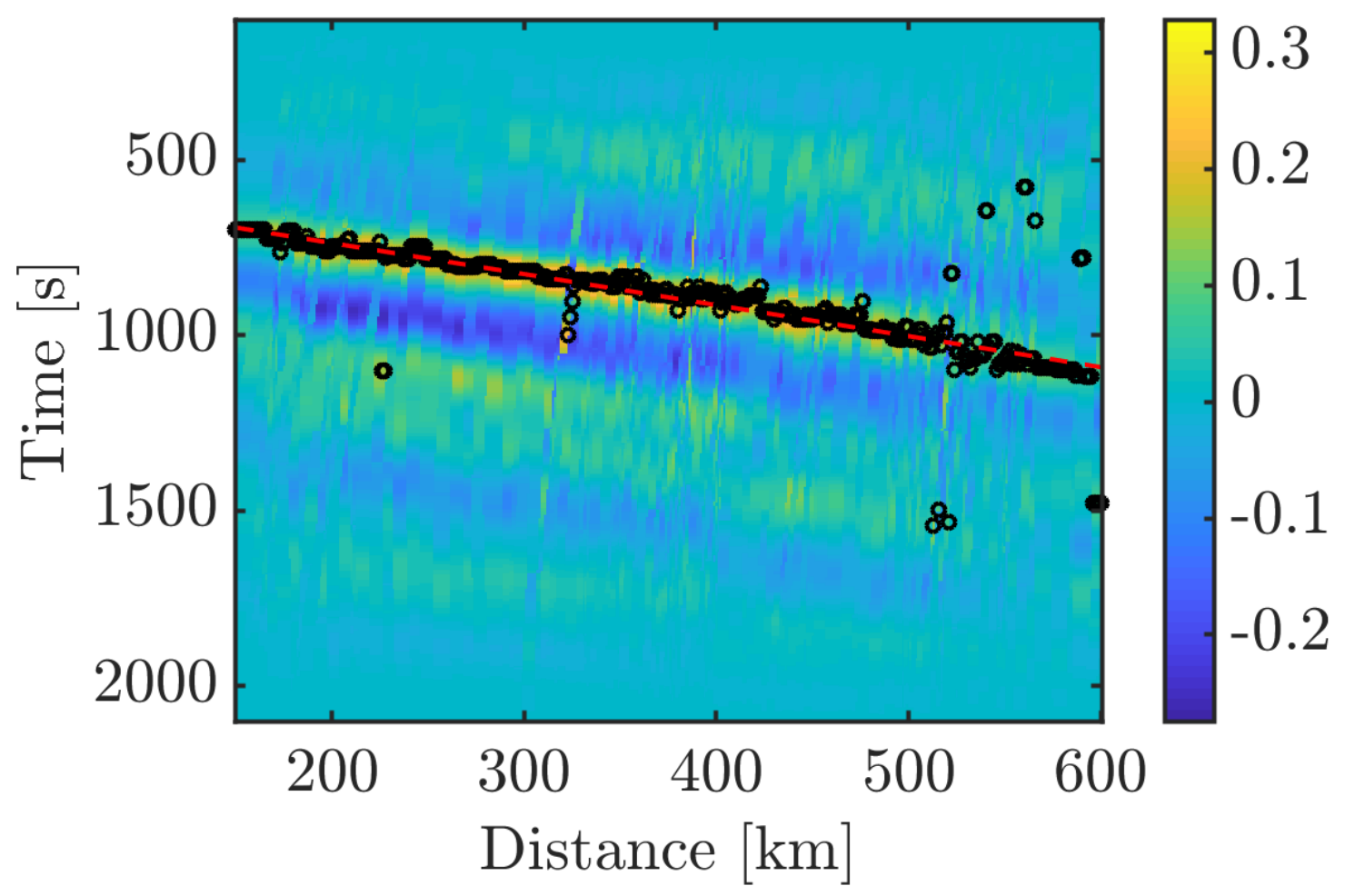

Figure B.1: Interpolated TEC and best fit line to the maximum amplitude TEC. 


\section{APPENDIX C:}

\section{ADDITIONAL SENSITIVITY EXAMPLES}


Additional plots from the sensitivity analysis.
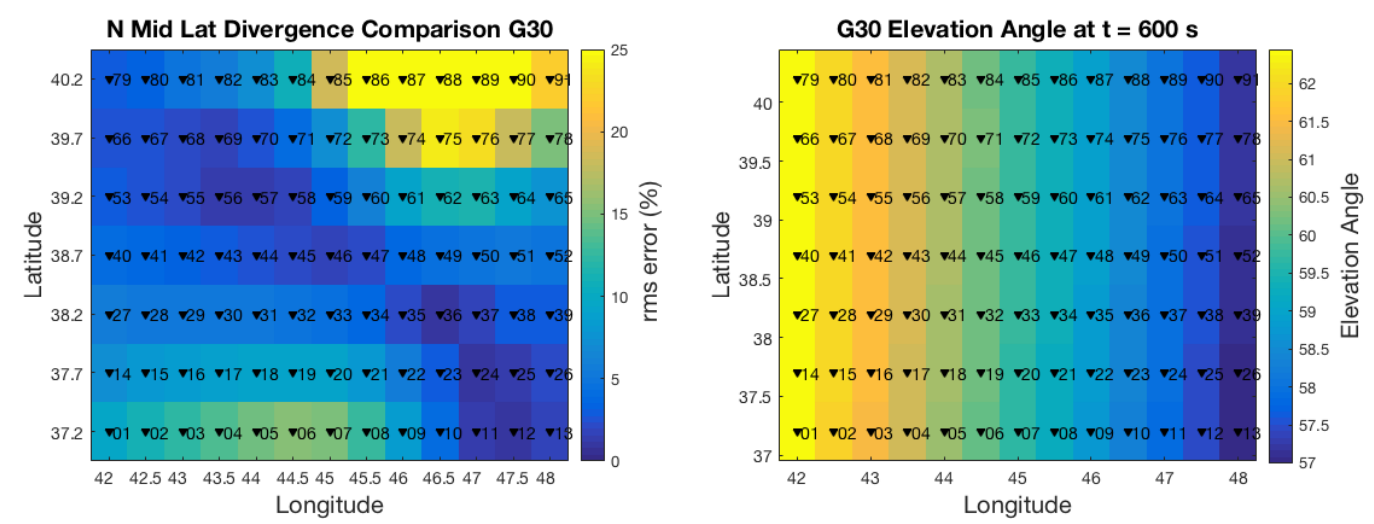

Figure C.1: Waveform differences and elevation angles for satellite G30 at N Mid Lat location. 

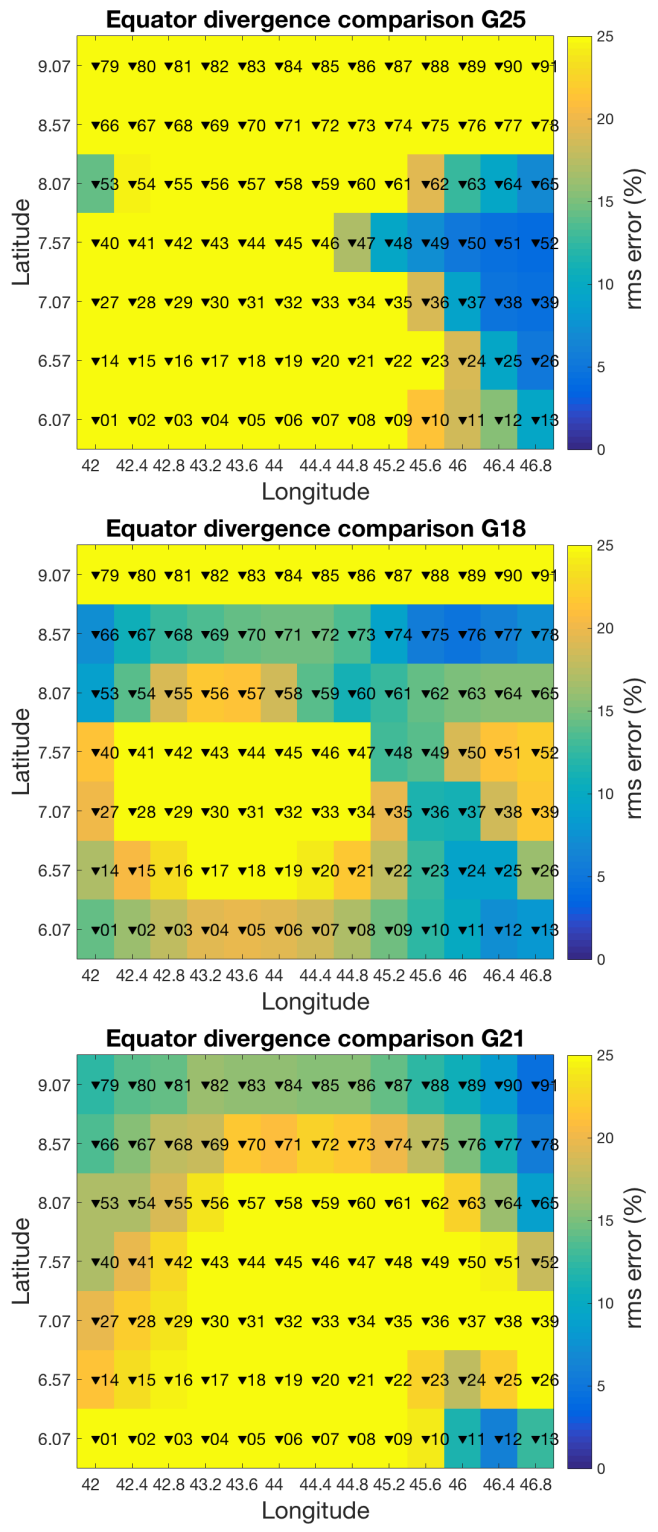

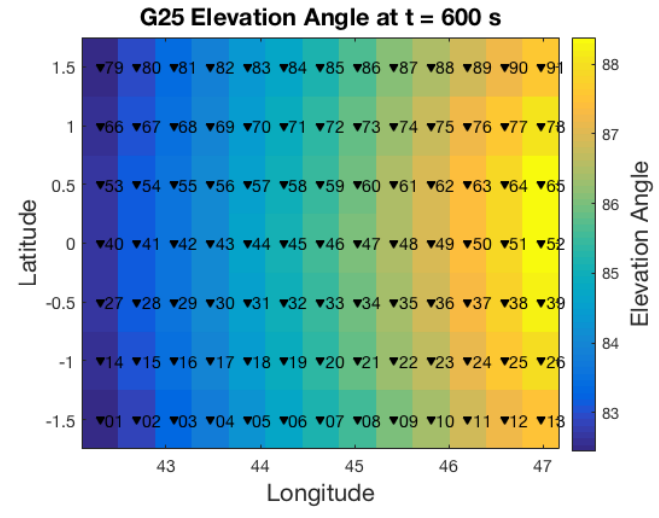

G18 Elevation Angle at $\mathrm{t}=600 \mathrm{~s}$
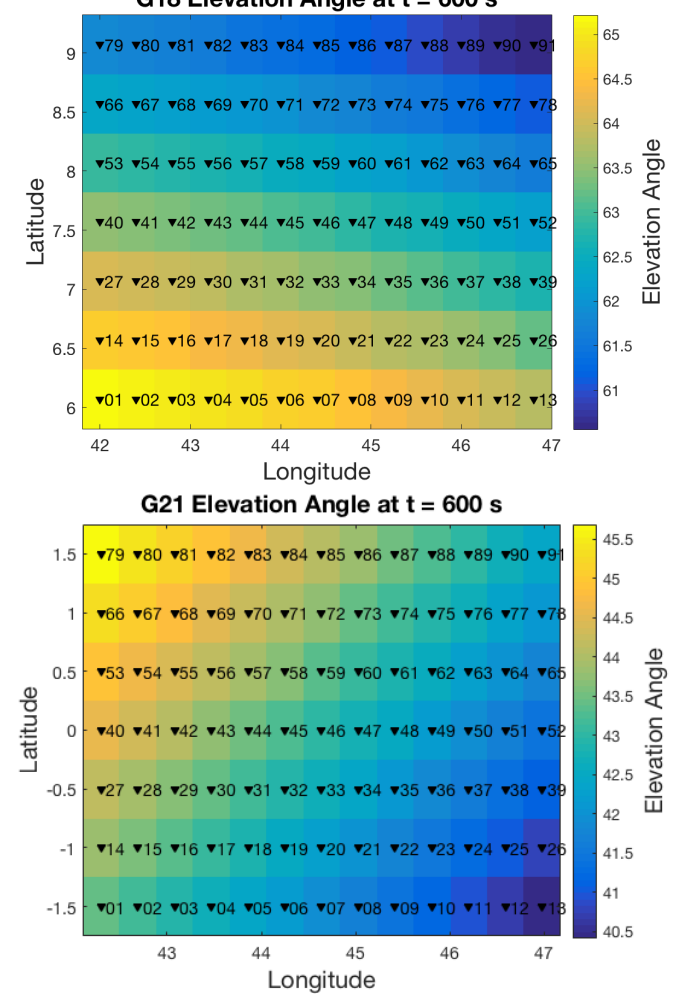

Figure C.2: Waveform differences and elevation angles for magnetic Equator. Latitude: $7.58^{\circ}$ Declination: $0.39^{\circ}$, Inclination $0.32^{\circ}$ 
APPENDIX D:

\section{KAIKOURA TEC OBSERVATIONS}


In this electronic supplement we present the TEC times series data in the same format as presented in the main article. In addition to G20 and R22, satellites G05, G13, G21 and G29 were visible to GNSS ground stations during the earthquake. We examined the TEC for these six satellites and show plots similar to Figure 5.5 and Figure 5.7 in Figures D.1-D.9. Satellites G20, R22 (discussed in main article), G21, and G29 demonstrate well-recorded TEC perturbations. We present all data that meet the amplitude selection criteria discussed in the main text. G21 is interesting in that it is the only satellite in which Epicenter 1 shows better coherence with IPP epicentral distance (Figure D.2). This is likely because this satellite samples a larger portion of the ionosphere to the west of the rupture area. However, at low elevation angles the IPP location is less-well resolved, and as a result the epicentral distance estimate is not as accurate as for other satellites. We observe this in the plot of the SNR, where even the modeled data show significant scatter compared to other satellites (right panel in Figure D.2). Satellites G5 and G13 have fewer stations with an obvious signal, and many of the stations at greater than $400 \mathrm{~km}$ (Figure D.4 and Figure D.5) have a low SNR (less than 4). Although stations close to the two epicenter locations have a clear signal, there are significant gaps between stations so it is difficult to determine conclusively which Epicenter location best matches the moveout.

We present TEC waveform comparisons for G21, G29 ,G13 and G5 in Figures D.6D.9. We present the IPP tracks for the waveforms presented in the right panel of each figure. The modeled signals are clear in G21 and G29, but the models vary in the fit, with the arrival times of Epicenter 2 matching the observed TEC better than Epicenter 1. At near epicentral distances, satellites G05 and G13 show likely TIDs, 
but because the SNR is low (e.g. Figures D.4 and Figure D.5), it is difficult to asses how well the modeled waveforms fit the observed TEC. Both satellites sample IPPs further to the East than the other satellites, and both satellites have long IPP tracks due to low elevation angles. This can explain why the TEC observations are limited to few stations.
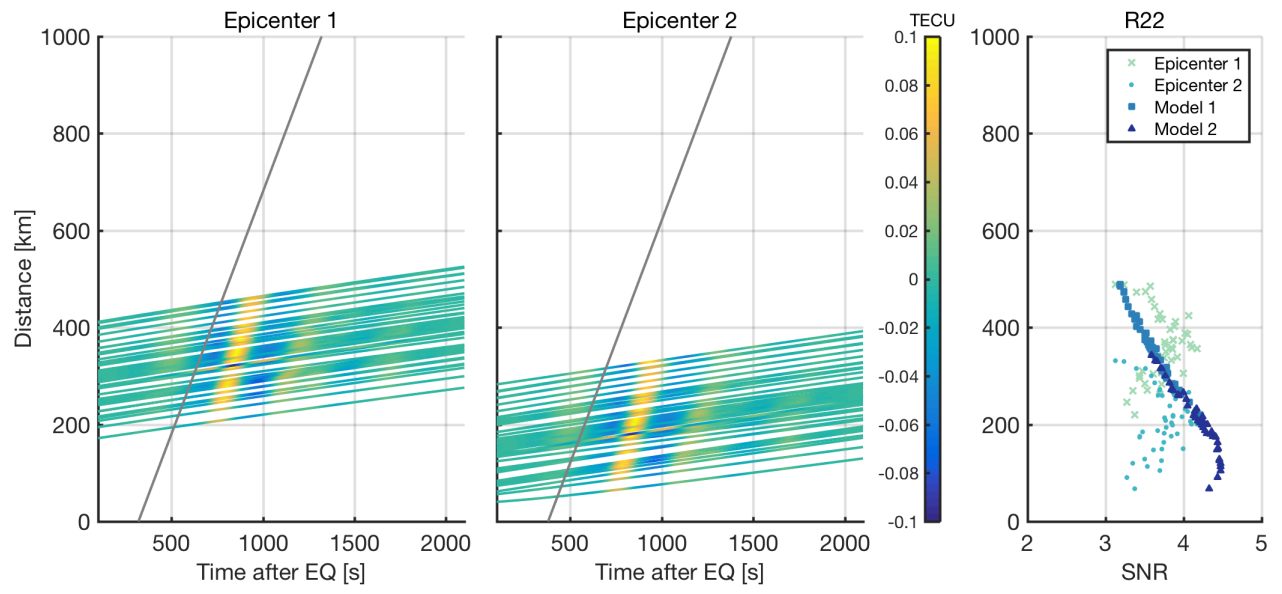

Figure D.1: TEC time series data for Satellite R22. Stations below the cutoff of 0.05 TECU have been removed.
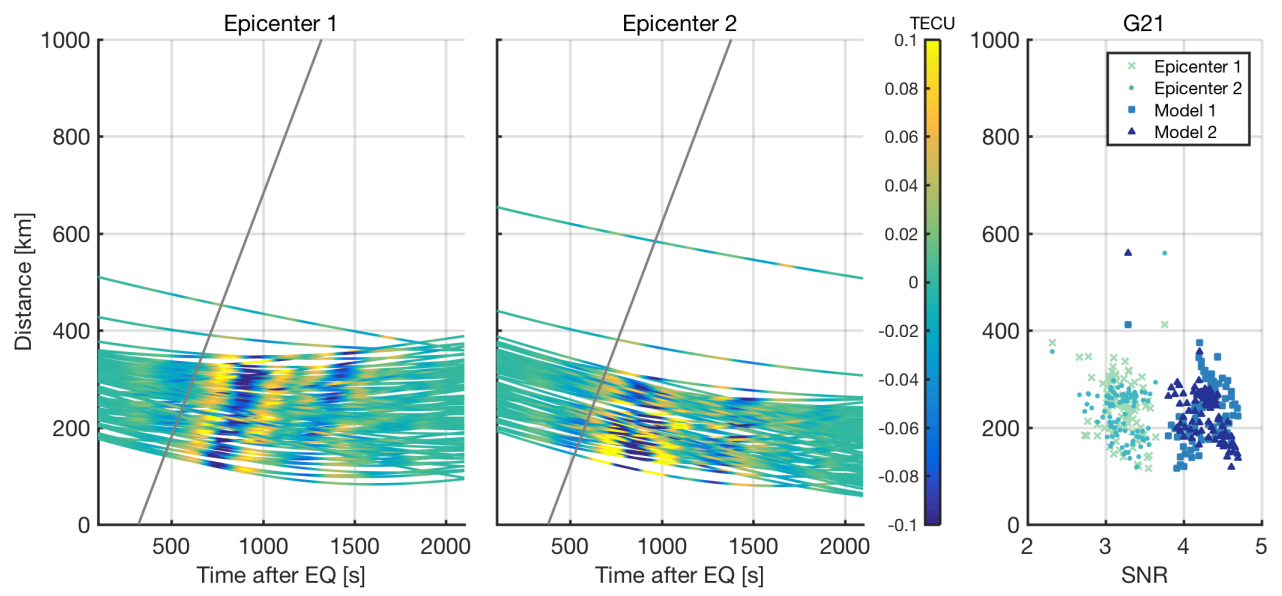

Figure D.2: TEC time series data for Satellite R21. Stations below the cutoff of 0.05 TECU have been removed. 

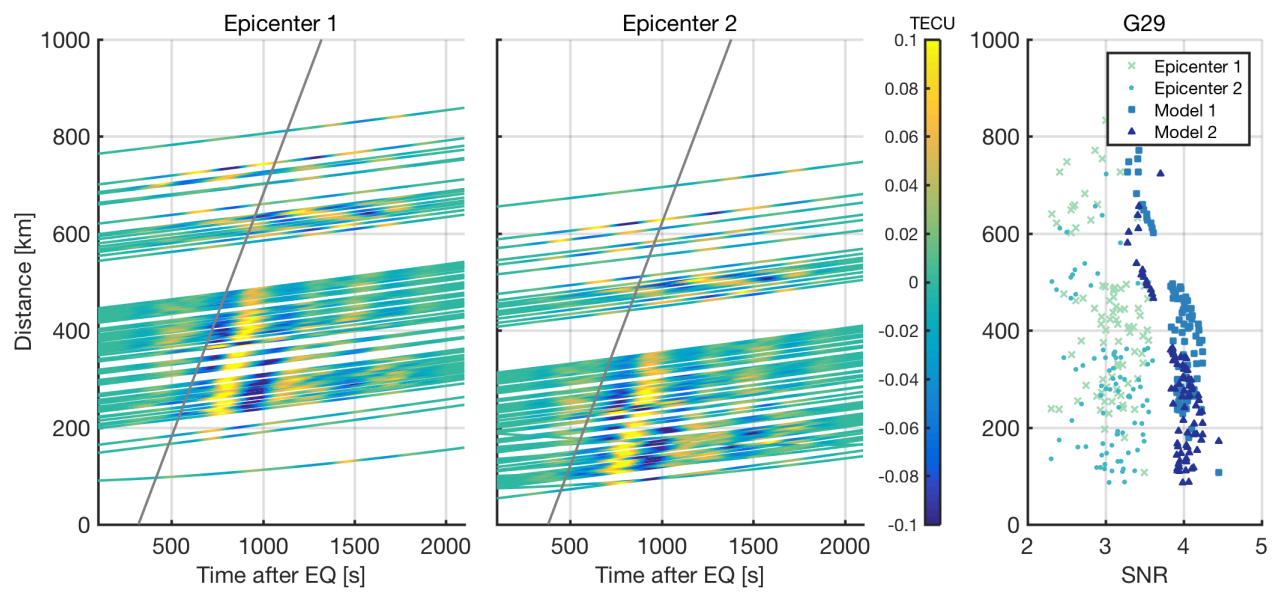

Figure D.3: TEC time series data for Satellite G29. Stations below the cutoff of 0.05 TECU have been removed.
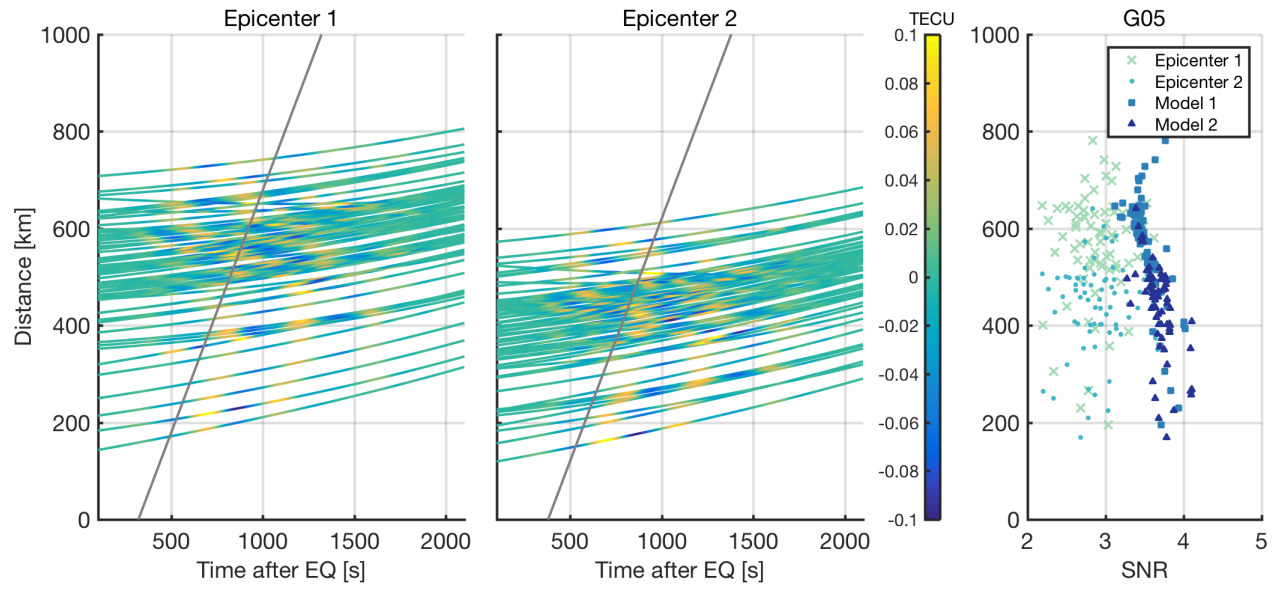

Figure D.4: TEC time series data for Satellite G05. Stations below the cutoff of 0.05 TECU have been removed. 

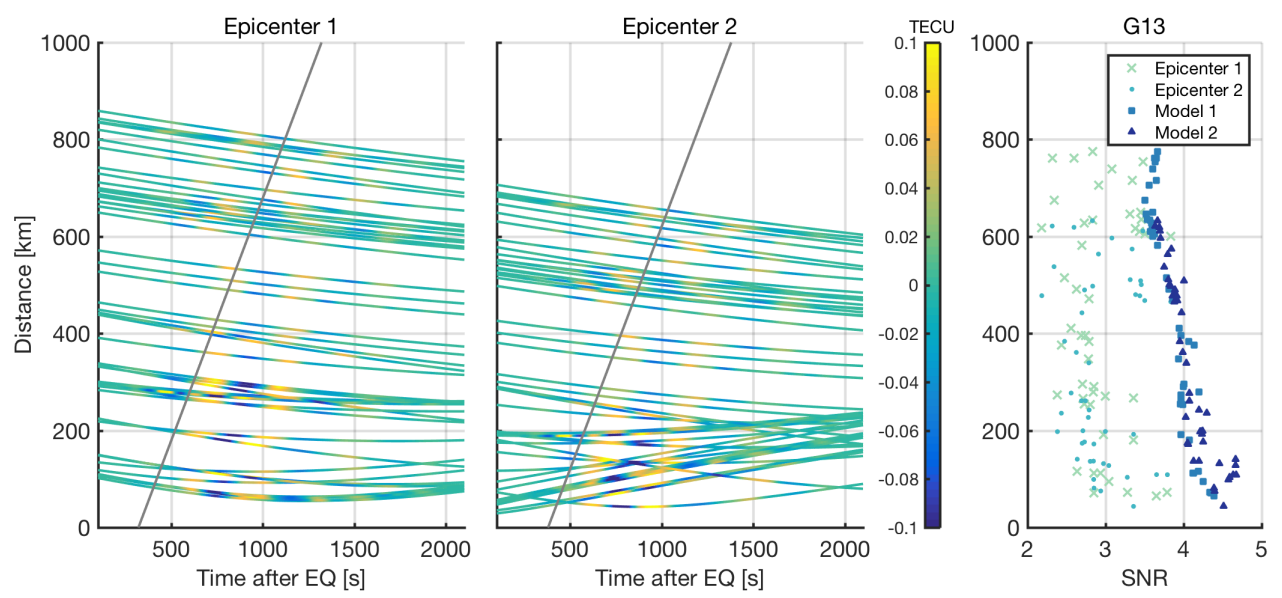

Figure D.5: TEC time series data for Satellite G13. Stations below the cutoff of 0.05 TECU have been removed.
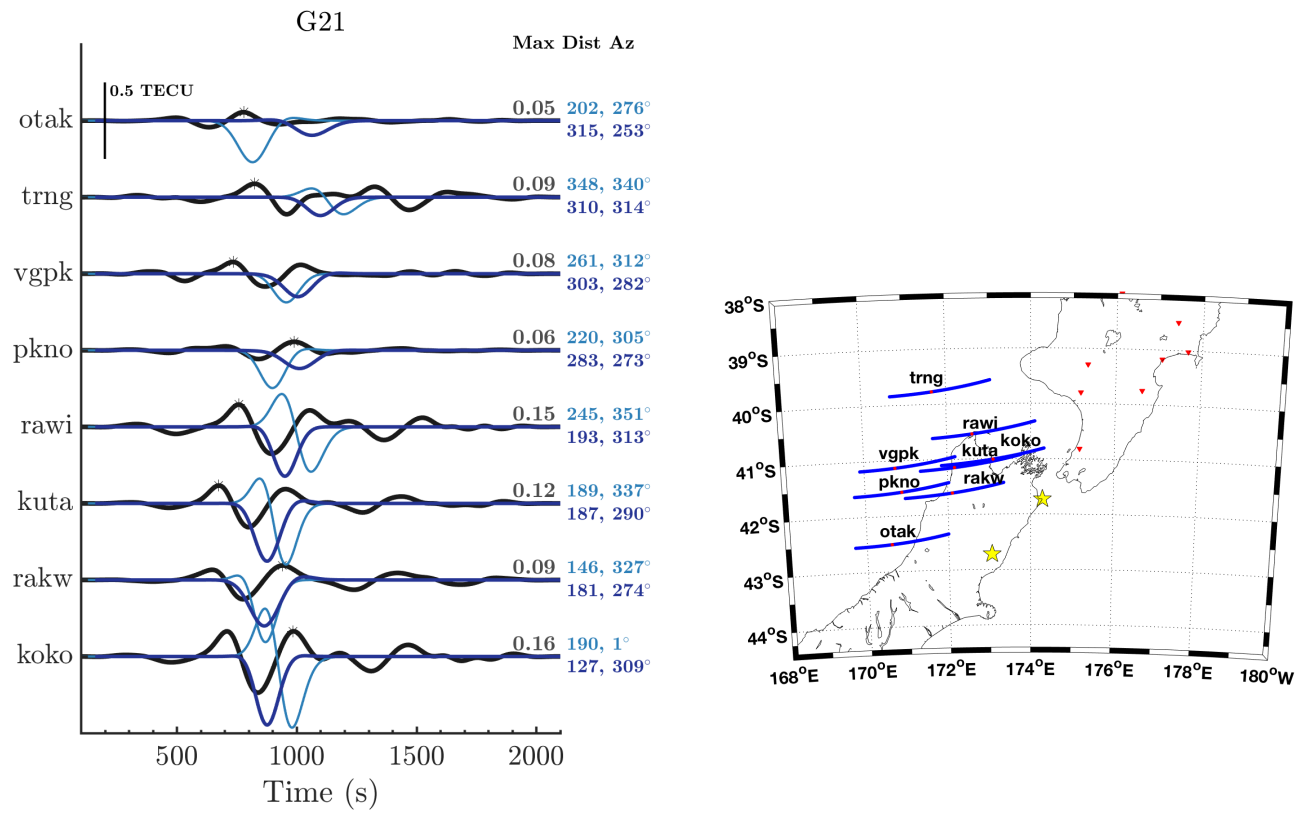

Figure D.6: Left: TEC waveform comparison for Satellite 21. Right: Map of IPP locations from the time of the initial rupture to 35 minutes after. Epicenters 1 and 2 are large stars and triangles are the stations on the ground. 


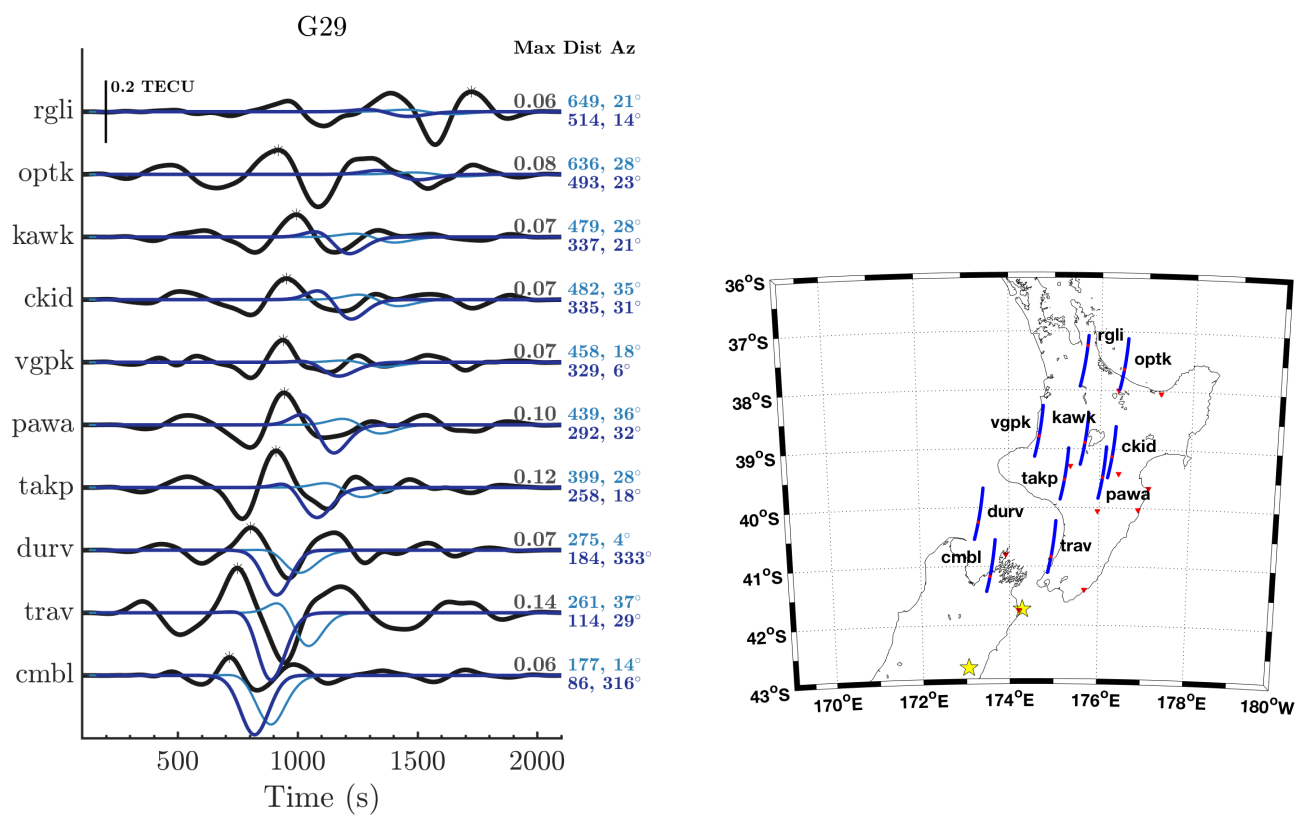

Figure D.7: Left: TEC waveform comparison for Satellite 29. Right: Map of IPP locations from the time of the initial rupture to 35 minutes after. Epicenters 1 and 2 are large stars and triangles are the stations on the ground. 


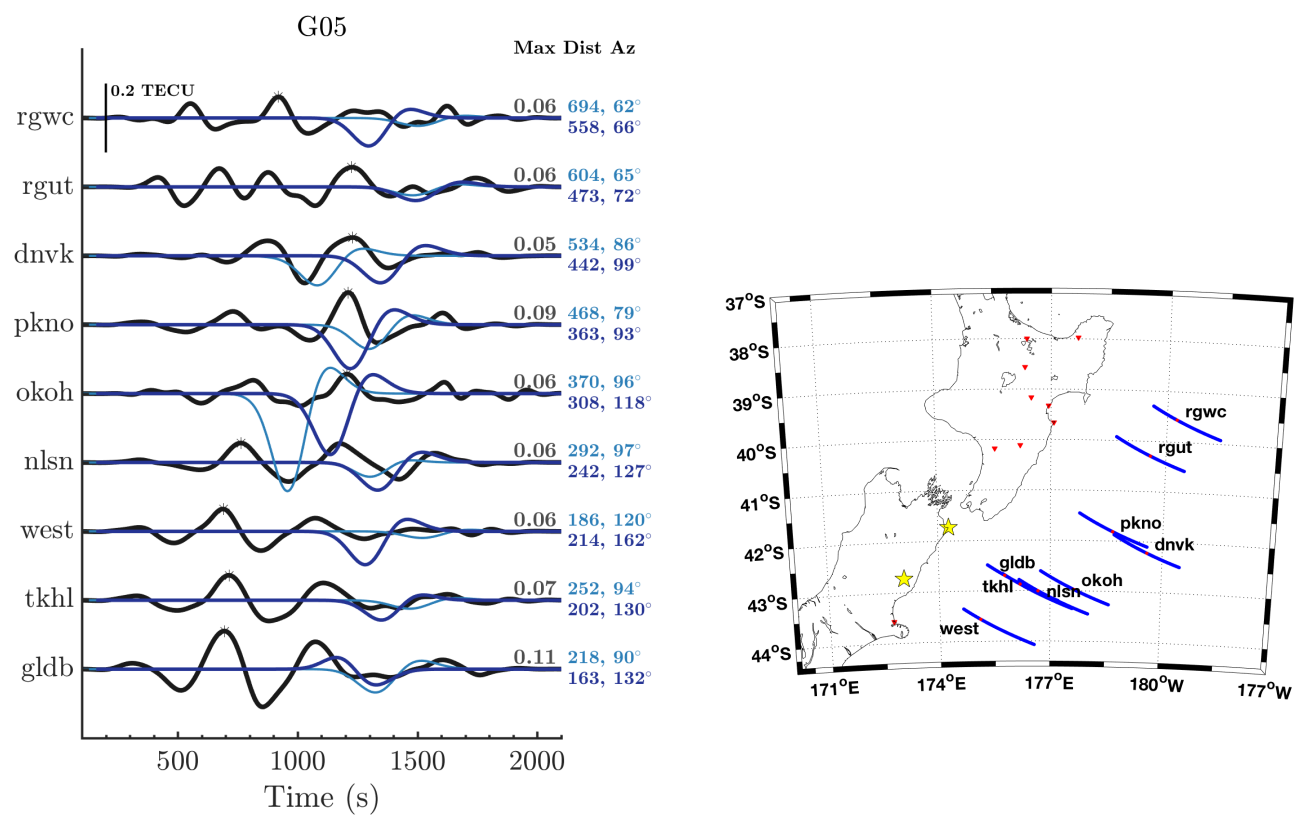

Figure D.8: Left: TEC waveform comparison for Satellite 05. Right: Map of IPP locations from the time of the initial rupture to 35 minutes after. Epicenters 1 and 2 are large stars and triangles are the stations on the ground. 


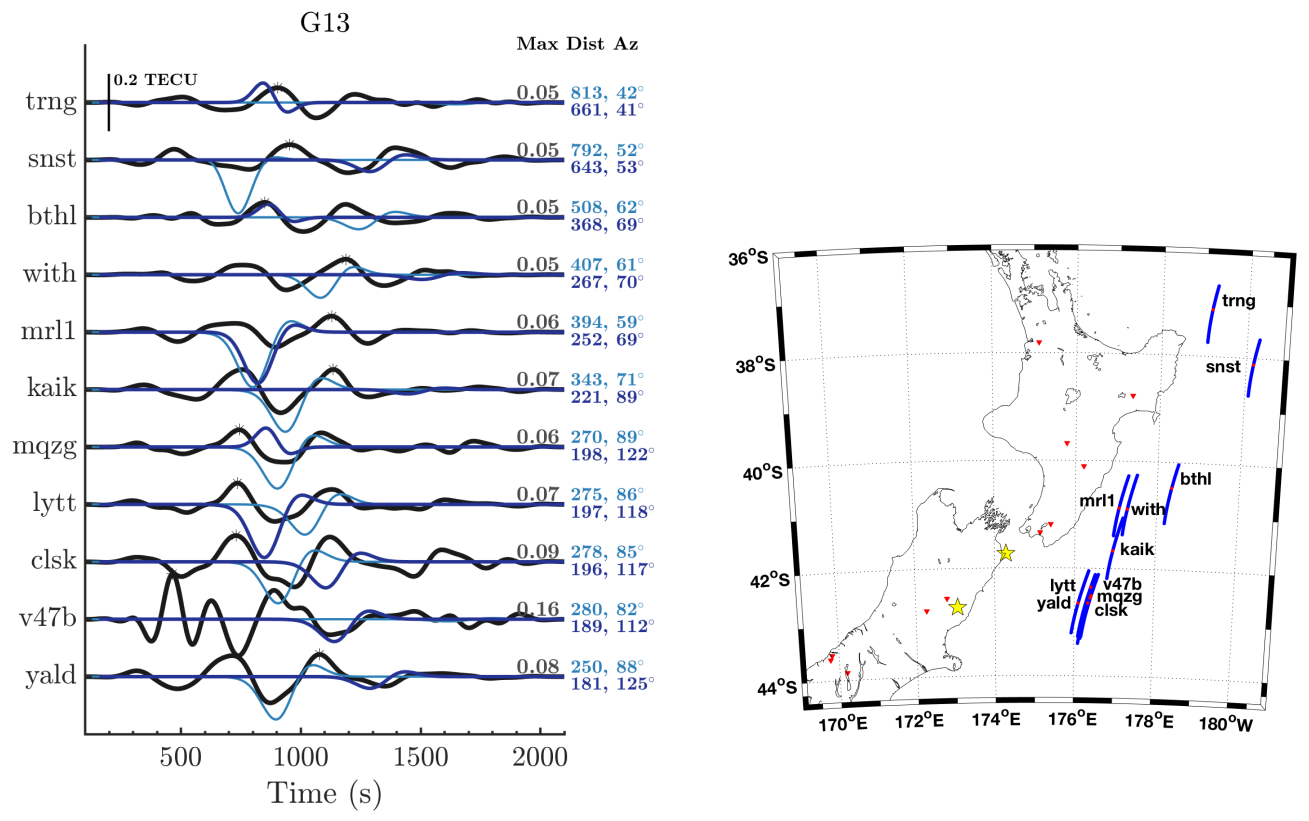

Figure D.9: Left: TEC waveform comparison for Satellite 13. Right: Map of IPP locations from the time of the initial rupture to 35 minutes after. Epicenters 1 and 2 are large stars and triangles are the stations on the ground. 